\title{
DECONFINED GLOBAL TYPES FOR ASYNCHRONOUS SESSIONS
}

\author{
FRANCESCO DAGNINO $\oplus^{a}$, PAOLA GIANNINI $\oplus^{b}$, AND MARIANGIOLA DEZANI-CIANCAGLINI $\oplus^{c}$ \\ ${ }^{a}$ DIBRIS, Università di Genova, Italy \\ e-mail address: francesco.dagnino@dibris.unige.it \\ ${ }^{b}$ DiSSTE, Università del Piemonte Orientale, Alessandria, Italy \\ e-mail address: paola.giannini@uniupo.it \\ ${ }^{c}$ Dipartimento di Informatica, Università di Torino, Italy \\ e-mail address: dezani@di.unito.it
}

\begin{abstract}
Multiparty sessions with asynchronous communications and global types play an important role for the modelling of interaction protocols in distributed systems. In designing such calculi the aim is to enforce, by typing, good properties for all participants, maximising, at the same time, the accepted behaviours. Our type system improves the state-of-the-art by typing all asynchronous sessions and preserving the key properties of Subject Reduction, Session Fidelity and Progress when some well-formedness conditions are satisfied. The type system comes together with a sound and complete type inference algorithm. The well-formedness conditions are undecidable, but an algorithm checking an expressive restriction of them recovers the effectiveness of typing.
\end{abstract}

\section{INTRODUCTION}

Multiparty sessions [HYC08, HYC16] are at the core of communication-based programming, since they formalise message exchange protocols. A key choice in the modelling is synchronous versus asynchronous communications, giving rise to synchronous and asynchronous multiparty sessions. In the multiparty session approach global types play the fundamental role of describing the whole scenario, while the behaviour of participants is implemented by processes. A natural question is when a set of processes agrees with a global type, meaning that participants behave according to the protocol described by the global type. The straightforward answer is the design of type assignment systems relating processes and global types. Typically, global types are projected onto participants to get the local behaviours prescribed by the protocol and then the processes implementing the participants are checked against such local behaviours. In conceiving such systems one wants to permit all possible typings which guarantee desirable properties: the mandatory Subject Reduction, but also Session Fidelity and Progress. Session Fidelity [HYC08, HYC16] means that the content and the order of exchanged messages respect the prescriptions of the global type.

This work was partially funded by the MUR project "T-LADIES" (PRIN 2020TL3X8X).

This original research has the financial support of the Università del Piemonte Orientale.

니밈

LOGICAL METHODS IN COMPUTER SCIENCE
DOI:10.46298/LMCS-19(1:3)2023

(c) F. Dagnino, P. Giannini, and M. Dezani-Ciancaglini

(c) Creative Commons 
Progress [DY11, CDCYP16] requires that all participants willing to communicate will be able to do it and, in case of asynchronous communication, also that all sent messages (which usually are in a queue) will be received.

A standard way of getting more permissive typings is through subtyping [GH05], which is used to compare local behaviours obtained by projection to the actual behaviours of participants. Following the substitution principle [LW94], we can safely put a process of some type where a process of a bigger type is expected. In the natural subtyping for synchronous multiparty sessions, bigger types have less inputs and more outputs [DH12]. This subtyping is not only correct, but also complete, that is, any extension of this subtyping would be unsound [GJP $\left.{ }^{+} 19\right]$. A powerful subtyping for asynchronous sessions was proposed in [MYH09] and recently proved to be complete $\left[\mathrm{GPP}^{+} 21\right]$. The key idea of this subtyping is the possibility of anticipating outputs before inputs to improve efficiency. This additional flexibility is justified by the fact that in an asynchronous setting outputs are non-blocking operations, hence they never prevent subsequent actions to be performed. An important issue of this subtyping is its undecidability [BCZ17, LY17], that makes the whole type assignment system undecidable. To overcome this problem, some decidable restrictions of this subtyping were proposed [BCZ17, LY17, BCZ18] and a sound, but necessarily not complete, decision algorithm is presented in $\left[\mathrm{BCL}^{+} 21\right]$.

Asynchronous communications better represent the exchange of messages between participants in different localities, and they are more suitable for implementations. So it is interesting to find alternatives to subtyping which increase typability of asynchronous multiparty sessions, and still ensure all desired properties. Recently a more permissive design of global types has been proposed [CDG21]. The key idea is to make the syntax of global types much closer to processes. This allows us to simplify the type assignment and to recover its decidability. Then, we study well-formedness conditions on global types ensuring the desired properties on processes. In other words, instead of directly deriving such properties from the syntax of global types through the type assignment, we split the problem in two steps: we first assign global types to networks and then transfer properties from types to processes through the typing relation. In this way, potential undecidability issues are confined to the second step, that is, they only depend on the complexity of the property one tries to enforce. In this way, the type assignment remains decidable and provides an abstraction from a local to a global perspective, which simplifies reasoning on global properties; however, by itself it does not ensure any of such properties.

More in detail, the formalism proposed in [CDG21] is based on the simple idea of splitting outputs and inputs in the syntax of global types, rather than modelling each communication action as a whole. In this way outputs can anticipate inputs, thus capturing their non-blocking nature directly at the level of global types. We dub "deconfined" such global types. The freedom gained by this definition is rather limited in [CDG21], whose main focus was to define an event structure semantics for asynchronous multiparty sessions. In particular, the well-formedness conditions that global types had to satisfy in [CDG21] still strongly confined their use.

In the present paper (which is the journal version of [DGD21]) we extend the syntax of global types in [CDG21, DGD21], allowing input choices for global types, and significantly enlarging the set of well-formed global types. In this way we are able to type also an example requiring a subtyping which fails for the algorithm in [BCL $\left.{ }^{+} 21\right]$. The idea is that the well-formedness of global types must guarantee that all participants waiting for a message are not stuck and that all sent messages find the corresponding readers. This 
last condition is particularly delicate for non-terminating computations in which the number of unread messages may grow indefinitely. Under this condition the type system enjoys Subject Reduction, Session Fidelity and Progress. Not surprisingly, the well-formedness of global types turns out to be undecidable, hence we design a decidable restriction to keep our system effective. The proposed algorithm extends similar ones presented in [CDG21, DGD21], adapting them to the more expressive syntax of our global types. In particular, we gain expressivity by:

- requiring that at least one input in a choice of inputs matches an output or a message in the queue;

- allowing an unbound number of unread messages when all of them will be eventually read.

We illustrate the proposed calculus with a running example in which the number of unread messages is unbounded. We choose this example since typing this session in standard type systems for multiparty sessions requires subtyping. Indeed, this is the running example of $\left[\mathrm{BCL}^{+} 21\right]$, where a decidable restriction of asynchronous subtyping is presented. In addition this example is typable neither in [CDG21] nor in [DGD21].

A hospital server $\mathrm{s}$ waits to receive from a patient $\mathrm{p}$ either some data $n d$ or a request to send her a report $p r$. In our calculus such a process is represented by $S=\mathrm{p} ?\left\{n d ; S_{1}, p r ; S_{1}\right\}$, where $\mathrm{p}$ ? means an input from participant $\mathrm{p}$ and the labels that can be read, in this case $n d$ and $p r$, are between curly brackets and followed by the process representing the remaining behaviour. After the reception of one of the two labels the server answers by sending either $o k$ or $k o$ and then it waits for another request. This is expressed by $S_{1}=\mathrm{p} !\{o k ; S, k o ; S\}$, where $\mathrm{p}$ ! means an output to participant $\mathrm{p}$ and again the two labels $o k$ and $k o$ are put between curly brackets. The patient, to save time, starts by sending some data and then waits for the response from the server, i.e., $P=\mathrm{s} ! n d ; P_{1}$ and $P_{1}=\mathrm{s} ?\{o k ; P, k o ; \mathrm{s} ! p r ; P\}$. If the patient receives $o k$ she continues sending next data. In case she receives $k o$ she sends the request for her report and then starts sending next data. So the multiparty session $\mathrm{p} \llbracket P \rrbracket\|\mathrm{s} \llbracket S \rrbracket\| \emptyset$, where $\emptyset$ is the empty queue, can execute as follows:

$$
\mathrm{p} \llbracket P \rrbracket\|\mathrm{s} \llbracket S \rrbracket\| \emptyset \stackrel{\mathrm{ps} ! n d}{\longrightarrow} \mathrm{p} \llbracket P_{1} \rrbracket\|\mathrm{s} \llbracket S \rrbracket\|\langle\mathrm{p}, n d, \mathrm{~s}\rangle \stackrel{\mathrm{ps} ? n d}{\longrightarrow} \mathrm{p} \llbracket P_{1} \rrbracket\left\|\mathrm{s} \llbracket S_{1} \rrbracket\right\| \emptyset
$$

decorating transition arrows with communications and denoting by $\langle\mathrm{p}, n d, \mathrm{~s}\rangle$ the message sent from $\mathrm{p}$ to $\mathrm{s}$ with label $n d$. The interaction may continue as shown in Figure 1 . If the server repeatedly responds $k o$ the queue can grow unboundedly. However, each message will be eventually read by the server.

The network $\mathrm{p} \llbracket P \rrbracket \| \mathrm{s} \llbracket S \rrbracket$ can be typed by the global type $\mathrm{G}=\mathrm{ps} ! n d ; \mathrm{G}_{1}$, where $\mathrm{G}_{1}=\mathrm{ps}\left\{\left\{n d ; \mathrm{G}_{2}, p r ; \mathrm{G}_{2}\right\}\right.$ and $\mathrm{G}_{2}=\mathrm{sp}$ ! $\{o k ; \mathrm{sp} ? o k ; \mathrm{G}, k o ; \mathrm{sp} ? k o ; \mathrm{ps} ! p r ; \mathrm{G}\}$. The type $\mathrm{G}$ prescribes that $\mathrm{p}$ put in the queue the label $n d$ and then $\mathrm{p}$ and $\mathrm{s}$ follow the protocol described by $G_{1}$. The type $G_{1}$ asks the server to read either the label $n d$ or the label $p r$ and in both cases the interaction follows the communications in $\mathrm{G}_{2}$.

Outline. Our calculus of multiparty sessions is presented in Section 2, where the Progress property is defined. Section 3 introduces our type system: we call it "wild" since each network can be typed in it. We define an LTS for global types with queues and we show Session Fidelity. Together with the type system, we give a sound and complete type inference algorithm proving that type inference is decidable. In Section 4 we tame global types to guarantee Subject Reduction and Progress. Unfortunately the balancing predicate, which ensures these properties, is undecidable, as shown in Section 5. The effectiveness of our type 


$$
\begin{array}{lll}
\mathrm{p} \llbracket P_{1} \rrbracket\left\|\mathrm{s} \llbracket S_{1} \rrbracket\right\| \emptyset & \stackrel{\mathrm{sp} ! k o}{\longrightarrow} & \mathrm{p} \llbracket P_{1} \rrbracket\|\mathrm{s} \llbracket S \rrbracket\|\langle\mathrm{s}, k o, \mathrm{p}\rangle \\
& \stackrel{\mathrm{s} \mathrm{p} ? k o}{\longrightarrow} & \mathrm{p} \llbracket \mathrm{s} ! p r ; P \rrbracket\|\mathrm{s} \llbracket S \rrbracket\| \emptyset \\
& \stackrel{\mathrm{ps} p r}{\longrightarrow} & \mathrm{p} \llbracket P \rrbracket\|\mathrm{s} \llbracket S \rrbracket\|\langle\mathrm{p}, p r, \mathrm{~s}\rangle \\
& \stackrel{\mathrm{ps} n d}{\longrightarrow} & \mathrm{p} \llbracket P_{1} \rrbracket\|\mathrm{s} \llbracket S \rrbracket\|\langle\mathrm{p}, p r, \mathrm{~s}\rangle \cdot\langle\mathrm{p}, n d, \mathrm{~s}\rangle \\
& \stackrel{\mathrm{ps} ? p r}{\longrightarrow} & \mathrm{p} \llbracket P_{1} \rrbracket\|\mathrm{s} \llbracket S 1 \rrbracket\|\langle\mathrm{p}, n d, \mathrm{~s}\rangle \\
& \stackrel{\mathrm{s} \mathrm{p} ! k o}{\longrightarrow} & \mathrm{p} \llbracket P_{1} \rrbracket\|\mathrm{s} \llbracket S \rrbracket\|\langle\mathrm{p}, n d, \mathrm{~s}\rangle \cdot\langle\mathrm{s}, k o, \mathrm{p}\rangle \\
& \stackrel{\mathrm{s} \mathrm{p} ? k o}{\longrightarrow} & \mathrm{p} \llbracket \mathrm{s} ! p r ; P \rrbracket\|\mathrm{s} \llbracket S \rrbracket\|\langle\mathrm{p}, n d, \mathrm{~s}\rangle \\
& \stackrel{\mathrm{ps} ! p r}{\longrightarrow} & \mathrm{p} \llbracket P \rrbracket\|\mathrm{s} \llbracket S \rrbracket\|\langle\mathrm{p}, n d, \mathrm{~s}\rangle \cdot\langle\mathrm{p}, p r, \mathrm{~s}\rangle \\
& \stackrel{\mathrm{ps} ! n d}{\longrightarrow} & \mathrm{p} \llbracket P_{1} \rrbracket\|\mathrm{s} \llbracket S \rrbracket\|\langle\mathrm{p}, n d, \mathrm{~s}\rangle \cdot\langle\mathrm{p}, p r, \mathrm{~s}\rangle \cdot\langle\mathrm{p}, n d, \mathrm{~s}\rangle
\end{array}
$$

Figure 1: A transition of the hospital session.

system is recovered in Section 6 by an algorithm that checks an inductive restriction of this predicate. Related and future works are discussed in Section 7.

\section{A Core Calculus for Multiparty Sessions}

Since our focus is on typing by means of global types, we only consider one multiparty session instead of many interleaved multiparty sessions. This allows us to depart from the standard syntax of processes with channels $\left[\mathrm{HYC08}, \mathrm{BCD}^{+} 08\right]$ in favour of simpler processes with output and input operators and explicit participants as in [DCGJ ${ }^{+} 16, \mathrm{SDC}_{19}$, GJP $\left.{ }^{+} 19\right]$.

We assume the following base sets: participants $\mathrm{p}, \mathrm{q}, \mathrm{r} \in$ Part, and labels $\lambda \in \mathrm{Lab}$.

Definition 2.1 (Processes). Processes $P$ are defined by:

$$
P \quad::=\rho \quad 0\left|\mathrm{p} !\left\{\lambda_{i} ; P_{i}\right\}_{i \in I}\right| \mathrm{p} ?\left\{\lambda_{i} ; P_{i}\right\}_{i \in I}
$$

where $I \neq \emptyset$ and $\lambda_{j} \neq \lambda_{h}$ for $j \neq h$.

The symbol $::={ }_{\rho}$, in the definition above and in other definitions, indicates that the productions should be interpreted coinductively. That is, they define possibly infinite processes. However, we assume such processes to be regular, i.e., with finitely many distinct subprocesses. In this way, we only obtain processes which are solutions of finite sets of equations, see [Cou83]. We choose this formulation as we will use coinduction in some definitions and proofs and, moreover, it allows us to avoid explicitly handling variables, thus simplifying a lot the technical development.

A process of shape $\mathrm{p} !\left\{\lambda_{i} ; P_{i}\right\}_{i \in I}$ (internal choice) chooses a label in the set $\left\{\lambda_{i} \mid i \in I\right\}$ to be sent to $\mathrm{p}$, and then behaves differently depending on the sent label. A process of shape $\mathrm{p} ?\left\{\lambda_{i} ; P_{i}\right\}_{i \in I}$ (external choice) waits for receiving one of the labels $\left\{\lambda_{i} \mid i \in I\right\}$ from $\mathrm{p}$, and then behaves differently depending on the received label. Note that the set of indexes in choices is assumed to be non-empty, and the corresponding labels to be all different. An internal choice which is a singleton is simply written $\mathrm{p} ! \lambda ; P$, analogously for an external choice. We omit traling $\mathbf{0}$ and we use $\mathrm{p} \dagger\left\{\lambda_{i} ; P_{i}\right\}_{i \in I}$ to denote either $\mathrm{p} !\left\{\lambda_{i} ; P_{i}\right\}_{i \in I}$ or $\mathrm{p} ?\left\{\lambda_{i} ; P_{i}\right\}_{i \in I}$.

In a full-fledged calculus, labels would carry values, namely they would be of shape $\lambda(v)$. For simplicity, here we consider pure labels. 


$$
\begin{aligned}
& \text { [SEND }] \quad \mathrm{p} \llbracket \mathrm{q} !\left\{\lambda_{i} ; P_{i}\right\}_{i \in I} \rrbracket\|\mathbb{N}\| \mathcal{M} \stackrel{\mathrm{pq} ! \lambda_{h}}{\longrightarrow} \mathrm{p} \llbracket P_{h} \rrbracket\|\mathbb{N}\| \mathcal{M} \cdot\left\langle\mathrm{p}, \lambda_{h}, \mathrm{q}\right\rangle \quad \text { where } h \in I \\
& {[\mathrm{RCV}] \quad \mathrm{q} \llbracket \mathrm{p} ?\left\{\lambda_{i} ; Q_{i}\right\}_{i \in I} \rrbracket\|\mathbb{N}\|\left\langle\mathrm{p}, \lambda_{h}, \mathrm{q}\right\rangle \cdot \mathcal{M} \stackrel{\mathrm{pq} ? \lambda_{h}}{\longrightarrow} \mathrm{q} \llbracket Q_{h} \rrbracket\|\mathbb{N}\| \mathcal{M} \quad \text { where } h \in I}
\end{aligned}
$$

Figure 2: LTS for asynchronous sessions.

Messages are triples $\langle\mathrm{p}, \lambda, \mathrm{q}\rangle$ denoting that participant $\mathrm{p}$ has sent label $\lambda$ to participant q. Sent messages are stored in a queue, from which they are subsequently fetched by the receiver.

Message queues $\mathcal{M}$ are defined by:

$$
\mathcal{M}::=\emptyset \mid\langle p, \lambda, q\rangle \cdot \mathcal{M}
$$

The order of messages in the queue is the order in which they will be read. Since order matters only between messages with the same sender and receiver, we always consider message queues modulo the following structural equivalence:

$$
\mathcal{M} \cdot\langle\mathrm{p}, \lambda, \mathrm{q}\rangle \cdot\left\langle\mathrm{r}, \lambda^{\prime}, \mathrm{s}\right\rangle \cdot \mathcal{M}^{\prime} \equiv \mathcal{M} \cdot\left\langle\mathrm{r}, \lambda^{\prime}, \mathrm{s}\right\rangle \cdot\langle\mathrm{p}, \lambda, \mathrm{q}\rangle \cdot \mathcal{M}^{\prime} \text { if } \mathrm{p} \neq \mathrm{r} \text { or } \mathrm{q} \neq \mathrm{s}
$$

Note, in particular, that $\langle\mathrm{p}, \lambda, \mathrm{q}\rangle \cdot\left\langle\mathrm{q}, \lambda^{\prime}, \mathrm{p}\right\rangle \equiv\left\langle\mathrm{q}, \lambda^{\prime}, \mathrm{p}\right\rangle \cdot\langle\mathrm{p}, \lambda, \mathrm{q}\rangle$. These two equivalent queues represent a situation in which both participants $\mathrm{p}$ and $\mathrm{q}$ have sent a label to the other one, and neither of them has read the message. This situation may happen in a multiparty session with asynchronous communication.

Multiparty sessions are comprised of networks, i.e. pairs participant/process of shape $\mathrm{p} \llbracket P \rrbracket$ composed in parallel, each with a different participant $\mathrm{p}$, and a message queue.

Definition 2.2 (Networks and multiparty sessions). (1) Networks are defined by

$\mathbb{N}::=\mathrm{p}_{1} \llbracket P_{1} \rrbracket\|\cdots\| \mathrm{p}_{n} \llbracket P_{n} \rrbracket$, where $n>0$ and $\mathrm{p}_{i} \neq \mathrm{p}_{j}$ for $i \neq j$.

(2) Multiparty sessions are defined by $\mathbb{N} \| \mathcal{M}$, where $\mathbb{N}$ is a network and $\mathcal{M}$ is a message queue.

In the following we use session as short for multiparty session.

We assume the standard structural congruence on sessions (denoted $\equiv$ ), that is, we consider sessions modulo permutation of components and adding/removing components of the shape $\mathrm{p} \llbracket \mathbf{0} \rrbracket$.

If $P \neq \mathbf{0}$ we write $\mathrm{p} \llbracket P \rrbracket \in \mathbb{N}$ as short for $\mathbb{N} \equiv \mathrm{p} \llbracket P \rrbracket \| \mathbb{N}^{\prime}$ for some $\mathbb{N}^{\prime}$. This abbreviation is justified by the associativity and commutativity of $\|$.

We define players $(\mathbb{N})=\{\mathrm{p} \mid \mathrm{p} \llbracket P \rrbracket \in \mathbb{N}\}$.

To define the asynchronous operational semantics of sessions, we use an LTS whose labels record the outputs and the inputs. To this end, communications (ranged over by $\beta$ ) are either the asynchronous emission of a label $\lambda$ from participant $\mathrm{p}$ to participant $\mathrm{q}$ (notation $\mathrm{pq} ! \lambda$ ) or the actual reading by participant $\mathrm{q}$ of the label $\lambda$ sent by participant $\mathrm{p}$ (notation $\mathrm{pq} ? \lambda)$.

The LTS semantics of sessions is specified by the two Rules [SEND] and [RCV] given in Figure 2. Rule [SEND] allows a participant $\mathrm{p}$ with an internal choice (a sender) to send one of its possible labels $\lambda_{h}$, by adding the corresponding message to the queue. Symmetrically, Rule $[\mathrm{RCV}]$ allows a participant $\mathrm{q}$ with an external choice (a receiver) to read the first message in the queue sent to her by a given participant $\mathrm{p}$, if its label $\lambda_{h}$ is one of those she is waiting for. 
The semantic property we aim to ensure, usually called progress [DY11, CDCYP16, $\mathrm{HLV}^{+}$16], is the conjunction of a safety property, deadlock-freedom, and two liveness properties: input lock-freedom and orphan message-freedom. Intuitively, a session is deadlock-free if, in every reachable state of computation, it is either terminated (i.e. of the shape $\mathbf{p} \llbracket \mathbf{0} \rrbracket \| \emptyset$ ) or it can move. It is input lock-free if every component wishing to do an input can eventually do so. Finally, it is orphan-message-free if every message stored in the queue is eventually read.

The following terminology and notational conventions are standard.

If $\mathbb{N}\left\|\mathcal{M} \stackrel{\beta_{1}}{\longrightarrow} \cdots \stackrel{\beta_{n}}{\longrightarrow} \mathbb{N}^{\prime}\right\| \mathcal{M}^{\prime}$ for some $n \geq 0$ (where by convention $\mathbb{N}^{\prime}\left\|\mathcal{M}^{\prime}=\mathbb{N}\right\| \mathcal{M}$ if $n=0$ ), then we say that $\mathbb{N}^{\prime} \| \mathcal{M}^{\prime}$ is a derivative of $\mathbb{N} \| \mathcal{M}$. We write $\mathbb{N} \| \mathcal{M} \stackrel{\beta}{\rightarrow}$ if $\mathbb{N}\left\|\mathcal{M} \stackrel{\beta}{\rightarrow} \mathbb{N}^{\prime}\right\| \mathcal{M}^{\prime}$ for some $\mathbb{N}^{\prime}, \mathcal{M}^{\prime}$.

Definition 2.3 (Live, terminated, deadlocked sessions). A session $\mathbb{N} \| \mathcal{M}$ is said to be

- live if $\mathbb{N} \| \mathcal{M} \stackrel{\beta}{\rightarrow}$ for some $\beta$;

- terminated if $\mathbb{N} \equiv \mathrm{p} \llbracket \mathbf{0} \rrbracket$ and $\mathcal{M}=\emptyset$;

- deadlocked if it is neither live nor terminated.

To formalise progress (Definition 2.6) we introduce another transition relation on sessions, which describes their lockstep execution: at each step, all components that are able to move execute exactly one asynchronous output or input.

We define the player of a communication as the sender in case of output and as the receiver in case of input:

$$
\operatorname{play}(\mathrm{pq} ! \lambda)=\mathrm{p} \quad \operatorname{play}(\mathrm{pq} ? \lambda)=\mathrm{q}
$$

Let $\Delta$ denote a non empty set of communications. We say that $\Delta$ is coherent for a session $\mathbb{N} \| \mathcal{M}$ if

(1) for all $\beta_{1}, \beta_{2} \in \Delta$, play $\left(\beta_{1}\right)=\operatorname{play}\left(\beta_{2}\right)$ implies $\beta_{1}=\beta_{2}$, and

(2) for all $\beta \in \Delta, \mathbb{N} \| \mathcal{M} \stackrel{\beta}{\rightarrow}$.

The lockstep transition relation $\mathbb{N}\left\|\mathcal{M} \triangleq \mathbb{N}^{\prime}\right\| \mathcal{M}^{\prime}$ is defined by:

$\mathbb{N}\left\|\mathcal{M} \triangleq \mathbb{N}^{\prime}\right\| \mathcal{M}^{\prime}$ if $\Delta=\left\{\beta_{1}, \ldots, \beta_{n}\right\}$ is a maximal coherent set for $\mathbb{N} \| \mathcal{M}$ and

$$
\mathbb{N}\left\|\mathcal{M} \stackrel{\beta_{1}}{\longrightarrow} \cdots \stackrel{\beta_{n}}{\longrightarrow} \mathbb{N}^{\prime}\right\| \mathcal{M}^{\prime}
$$

The notion of derivative can be reformulated for lockstep computations as follows.

If $\mathbb{N}\left\|\mathcal{M} \stackrel{\Delta_{1}}{\Longrightarrow} \cdots \stackrel{\Delta_{n}}{\Longrightarrow} \mathbb{N}^{\prime}\right\| \mathcal{M}^{\prime}$ for some $n \geq 0$ (where by convention $\mathbb{N}^{\prime}\left\|\mathcal{M}^{\prime}=\mathbb{N}\right\| \mathcal{M}$ if $n=0$ ), then we say that $\mathbb{N}^{\prime} \| \mathcal{M}^{\prime}$ is a lockstep derivative of $\mathbb{N} \| \mathcal{M}$. Clearly each lockstep derivative is a derivative, but not vice versa.

A lockstep computation is an either finite or infinite sequence of lockstep transitions, and it is complete if either it is finite and cannot be extended (because the last session is not live), or it is infinite. Let $\gamma$ range over lockstep computations.

Formally, a lockstep computation $\gamma$ can be denoted as follows, where $x \in \mathbf{N} \cup\{\omega\}$ is the length of $\gamma$ :

$$
\gamma=\left\{\mathbb{N}_{k}\left\|\mathcal{M}_{k} \stackrel{\Delta_{k}}{\underset{k}{\rightleftarrows}} \mathbb{N}_{k+1}\right\| \mathcal{M}_{k+1}\right\}_{k<x}
$$

That is, $\gamma$ is represented as the set of its successive lockstep transitions, where the arrow subscript $k$ is used to indicate that the transition occurs in the $k$-th step of the computation. This is needed in order to distinguish equal transitions occurring in different steps. For 
instance, in the session $\mathbb{N} \|\langle\mathrm{p}, \lambda, \mathrm{q}\rangle$, where $\mathbb{N}=\mathrm{p} \llbracket P \rrbracket \| \mathrm{q} \llbracket Q \rrbracket$ with $P=\mathrm{q} ! \lambda ; P$ and $Q=\mathrm{p} ? \lambda ; Q$, all lockstep transitions with $k \geq 1$ are of the form

$$
\mathbb{N}\|\langle\mathrm{p}, \lambda, \mathrm{q}\rangle \stackrel{\{\mathrm{pq} ! \lambda, \mathrm{pq} ? \lambda\}}{\underset{k}{\longrightarrow}} \mathbb{N}\|\langle\mathrm{p}, \lambda, \mathrm{q}\rangle
$$

We can now formalise the progress property:

Definition 2.4 (Input-enabling session). A session $\mathbb{N} \| \mathcal{M}$ is input-enabling if $\mathrm{p} \llbracket \mathrm{q} ?\left\{\lambda_{i} ; P_{i}\right\}_{i \in I} \rrbracket \in$ $\mathbb{N}$ implies that, for all complete

$$
\gamma=\left\{\mathbb{N}_{k}\left\|\mathcal{M}_{k} \stackrel{\Delta_{k}}{\underset{k}{\longrightarrow}} \mathbb{N}_{k+1}\right\| \mathcal{M}_{k+1}\right\}_{k<x}
$$

with $\mathbb{N}_{0}\left\|\mathcal{M}_{0}=\mathbb{N}\right\| \mathcal{M}$, there exists $h<x$ such that pq? $\lambda_{j} \in \Delta_{h}$ for some $j \in I$.

Definition 2.5 (Queue-consuming session). A session $\mathbb{N} \| \mathcal{M}$ is queue-consuming if $\mathcal{M} \equiv$ $\langle\mathrm{p}, \lambda, \mathrm{q}\rangle \cdot \mathcal{M}^{\prime}$ implies that, for all complete

$$
\gamma=\left\{\mathbb{N}_{k}\left\|\mathcal{M}_{k} \stackrel{\Delta_{k}}{\underset{k}{\longrightarrow}} \mathbb{N}_{k+1}\right\| \mathcal{M}_{k+1}\right\}_{k<x}
$$

with $\mathbb{N}_{0}\left\|\mathcal{M}_{0}=\mathbb{N}\right\| \mathcal{M}$, there exists $h<x$ such that pq? $\lambda \in \Delta_{h}$.

Definition 2.6 (Progress). A session has the progress property if:

(1) (Deadlock-freedom) None of its lockstep derivatives is deadlocked;

(2) (No locked inputs) All its lockstep derivatives are input-enabling;

(3) (No orphan messages) All its lockstep derivatives are queue-consuming.

It is easy to see that deadlock-freedom implies no locked inputs and no orphan messages for finite computations.

Example 2.7. Let $\mathbb{N}=\mathrm{p} \llbracket P \rrbracket\|\mathrm{q} \llbracket Q \rrbracket\| \mathrm{r} \llbracket R \rrbracket$, where $P=\mathrm{q} ! \lambda ; P, Q=\mathrm{p} ? \lambda ; \mathrm{r} ? \lambda^{\prime} ; Q$ and $R=\mathrm{q} ! \lambda^{\prime} ; R$.

The unique complete lockstep computation of $\mathbb{N} \| \emptyset$ is the following:

$$
\begin{aligned}
& \mathbb{N}\left\|\emptyset \stackrel{\left\{p q ! \lambda, r q ! \lambda^{\prime}\right\}}{\longrightarrow} \quad \mathbb{N}\right\|\langle p, \lambda, q\rangle \cdot\left\langle r, \lambda^{\prime}, q\right\rangle \\
& \stackrel{\left\{\mathrm{pq} ! \lambda, \mathrm{pq} ? \lambda, \mathrm{rq} ! \lambda^{\prime}\right\}}{\longrightarrow} \mathrm{p} \llbracket P \rrbracket\left\|\mathrm{q} \llbracket \mathrm{r} ? \lambda^{\prime} ; Q \rrbracket\right\| \mathrm{r} \llbracket R \rrbracket \|\left\langle\mathrm{r}, \lambda^{\prime}, \mathrm{q}\right\rangle \cdot\langle\mathrm{p}, \lambda, \mathrm{q}\rangle \cdot\left\langle\mathrm{r}, \lambda^{\prime}, \mathrm{q}\right\rangle \\
& \stackrel{\left\{\mathrm{pq} ! \lambda, \mathrm{rq} ? \lambda^{\prime}, \mathrm{rq} ! \lambda^{\prime}\right\}}{\longrightarrow} \mathbb{N} \|\langle\mathrm{p}, \lambda, \mathrm{q}\rangle \cdot\left\langle\mathrm{r}, \lambda^{\prime}, \mathrm{q}\right\rangle \cdot\langle\mathrm{p}, \lambda, \mathrm{q}\rangle \cdot\left\langle\mathrm{r}, \lambda^{\prime}, \mathrm{q}\right\rangle \\
& \text {.. } \ldots
\end{aligned}
$$

It is easy to check that $\mathbb{N} \| \emptyset$ has the progress property. Indeed, every input communication in $Q$ is eventually enabled, and, even though the queue grows at each step of the lockstep computation, every message in the queue is eventually read.

\section{A Wild Type System}

In this section we first present global types and the type system for networks, then we give an LTS for global types in parallel with queues which allows us to get Session Fidelity. Lastly, we present a sound and complete type inference algorithm. We call wild this type system since the freedom in the syntax of global types allows us to type all networks, see Theorem 3.8. 


$$
\begin{gathered}
{[\text { End }] \overline{\overline{\text { End } \vdash \mathrm{p} \llbracket \mathbf{0} \rrbracket}}} \\
{[\text { Out }] \frac{\mathrm{G}_{i} \vdash \mathrm{p} \llbracket P_{i} \rrbracket \| \mathbb{N} \text { players }\left(\mathrm{G}_{i}\right) \backslash\{\mathrm{p}\}=\operatorname{players}(\mathbb{N}) \quad \forall i \in I}{\mathrm{pq} !\left\{\lambda_{i} ; \mathrm{G}_{i}\right\}_{i \in I} \vdash \mathrm{p} \llbracket \mathrm{q} !\left\{\lambda_{i} ; P_{i}\right\}_{i \in I} \rrbracket \| \mathbb{N}}} \\
{[\mathrm{IN}] \frac{\mathrm{G}_{i} \vdash \mathrm{p} \llbracket P_{i} \rrbracket \| \mathbb{N} \text { players }\left(\mathrm{G}_{i}\right) \backslash\{\mathrm{p}\}=\operatorname{players}(\mathbb{N}) \quad \forall i \in I}{\mathrm{q} \mathrm{p} ?\left\{\lambda_{i} ; \mathrm{G}_{i}\right\}_{i \in I} \vdash \mathrm{p} \llbracket \mathrm{q} ?\left\{\lambda_{j} ; P_{j}\right\}_{j \in J} \rrbracket \| \mathbb{N}} \quad I \subseteq J}
\end{gathered}
$$

Figure 3: Typing rules for networks.

3.1. Global types. Our global types can be obtained from the standard ones [HYC08, HYC16] by splitting output and input communications. For this reason we call our global types deconfined.

Definition 3.1 (Global types). Global types $\mathrm{G}$ are defined by the following grammar:

$$
\mathrm{G}::=\rho \text { End }\left|\mathrm{pq} !\left\{\lambda_{i} ; \mathrm{G}_{i}\right\}_{i \in I}\right| \mathrm{pq} ?\left\{\lambda_{i} ; \mathrm{G}_{i}\right\}_{i \in I}
$$

where $I \neq \emptyset, \mathrm{p} \neq \mathrm{q}$ and $\lambda_{j} \neq \lambda_{h}$ for $j \neq h$.

As for processes, $::=\rho$ indicates that global types are regular.

The global type $\mathrm{pq} !\left\{\lambda_{i} ; \mathrm{G}_{i}\right\}_{i \in I}$ specifies that player $\mathrm{p}$ sends a label $\lambda_{k}$ with $k \in I$ to participant $\mathrm{q}$ and then the interaction described by the global type $\mathrm{G}_{k}$ takes place. The global type pq? $\left\{\lambda_{i} ; \mathrm{G}_{i}\right\}_{i \in I}$ specifies that player q receives a label $\lambda_{k}$ with $k \in I$ from participant $\mathrm{p}$ and then the interaction described by the global type $\mathrm{G}_{k}$ takes place. An output choice which is a singleton is simply written $p q ! \lambda ; G$ and similarly for an input choice. We omit trailing End and we use pq $\dagger\left\{\lambda_{i} ; \mathrm{G}_{i}\right\}_{i \in I}$ to denote either pq! $\left\{\lambda_{i} ; \mathrm{G}_{i}\right\}_{i \in I}$ or pq? $\left\{\lambda_{i} ; \mathrm{G}_{i}\right\}_{i \in I}$.

We define play $\left(\mathrm{pq} !\left\{\lambda_{i} ; \mathrm{G}_{i}\right\}_{i \in I}\right)=\mathrm{p}$ and play $\left(\mathrm{pq} ?\left\{\lambda_{i} ; \mathrm{G}_{i}\right\}_{i \in I}\right)=\mathrm{q}$. The set of players of a global type, notation players $(\mathrm{G})$, is the smallest set of participants satisfying the following equations:

$$
\begin{array}{ll}
\operatorname{players}(\mathrm{End}) & =\emptyset \\
\operatorname{players}\left(\mathrm{p} \mathrm{q} !\left\{\lambda_{i} ; \mathrm{G}_{i}\right\}_{i \in I}\right) & =\{\mathrm{p}\} \cup \bigcup_{i \in I} \operatorname{players}\left(\mathrm{G}_{i}\right) \\
\text { players }\left(\mathrm{pq} ?\left\{\lambda_{i} ; \mathrm{G}_{i}\right\}_{i \in I}\right) & =\{\mathrm{q}\} \cup \bigcup_{i \in I} \operatorname{players}\left(\mathrm{G}_{i}\right)
\end{array}
$$

Notice that the sets of players are always finite thanks to the regularity of global types.

Global types are an abstraction of networks, usually described by projecting global types to local types which are assigned to processes. The simplicity of our calculus and the flexibility of our global types allow us to formulate a type system deriving directly global types for networks, judgments $\mathrm{G} \vdash \mathbb{N}$, see Figure 3. The double line here and in the following indicates that the rules are coinductive. Rules [OUT] and [IN] just add simultaneously outputs and inputs to global types and to corresponding processes inside networks. Rule [OUT] requires the same outputs in the process and in the global type, while the subtyping for session types [DH12] allows less outputs in the process than in the global type. The only consequence of our choice is that less global types are derivable for a given network. The gain is a formulation of Session Fidelity ensuring that networks implement corresponding global types, see Theorem 3.9. Instead Rule [IN] allows more inputs in the process than in the global type, just mimicking the subtyping for session types [DH12].

The condition players $\left(\mathrm{G}_{i}\right) \backslash\{\mathrm{p}\}=$ players $(\mathbb{N})$ for all $i \in I$ ensures that the players of $\mathbb{N}$ and of $\mathrm{G}$ coincide whenever $\mathrm{G} \vdash \mathbb{N}$, as stated in the following lemma, whose proof is straightforward. 


$$
\frac{\mathcal{D}}{\frac{\mathcal{D}}{\overline{\mathrm{G}_{1} \vdash \mathrm{p} \llbracket P_{1} \rrbracket \| \mathrm{s} \llbracket S \rrbracket}}[\mathrm{OUT}]}[\mathrm{IN}]
$$

where $\mathcal{D}$ is the following derivation



Figure 4: A typing of the hospital network $\mathrm{p} \llbracket P \rrbracket \| \mathrm{s} \llbracket S \rrbracket$, where $P=\mathrm{s} ! n d ; P_{1}, P_{1}=$ $\mathrm{s} ?\{o k ; P, k o ; \mathrm{s} ! p r ; P\}, S=\mathrm{p} ?\left\{n d ; S_{1}, p r ; S_{1}\right\}, S_{1}=\mathrm{p} !\{o k ; S, k o ; S\}$ with the global type $\mathrm{G}=\operatorname{ps} ! n d ; \mathrm{G}_{1}$, where $\mathrm{G}_{1}=\mathrm{ps} ?\left\{n d ; \mathrm{G}_{2}, \operatorname{pr} ; \mathrm{G}_{2}\right\}$ and $\mathrm{G}_{2}=$ sp! $\{o k ; \mathrm{sp} ? o k ; \mathrm{G}, k o ; \mathrm{sp} ? k o ; \mathrm{ps}$ ! pr; G $\}$.

Lemma 3.2. If $\mathrm{G} \vdash \mathbb{N}$, then players $(\mathrm{G})=\operatorname{players}(\mathbb{N})$.

This lemma forbids for example to derive $\mathrm{G} \vdash \mathrm{p} \llbracket P \rrbracket \| \mathrm{q} \llbracket Q \rrbracket$ with $\mathrm{G}=\mathrm{p} \mathrm{q} ! \lambda ; \mathrm{G}$ and $P=\mathrm{q} ! \lambda ; P$ and $\mathbb{N}=\mathrm{q} \llbracket Q \rrbracket$ and $Q$ arbitrary, since $\mathrm{q}$ is not a player of $\mathrm{G}$. Anyway the given type system is wild since it allows us to type networks with non-matching inputs and outputs, as shown in Example 3.3. This motivates the well-formedness conditions on global types we will discuss in Section 4.

Example 3.3. We can derive $p q ! \lambda ; p q ? \lambda^{\prime} \vdash p \llbracket q ! \lambda \rrbracket \| q \llbracket p ? \lambda^{\prime} \rrbracket$ and this network in parallel with the empty queue evolves to $q \llbracket p ? \lambda^{\prime} \rrbracket \|\langle p, \lambda$, q $\rangle$ which is deadlocked.

Example 3.4. The typing of the running example discussed in the Introduction is the content of Figure 4. Notice that the premises of the application of Rule [IN] with conclusion $\mathrm{G}_{1} \vdash \mathrm{p} \llbracket P_{1} \rrbracket \| \mathrm{s} \llbracket S \rrbracket$ are two occurrences of the derivation $\mathcal{D}$, since the process $S$ is $\mathrm{p} ?\left\{n d ; S_{1}, p r ; S_{1}\right\}$.

The regularity of processes and global types ensures the decidability of type checking.

We now show that our type system allows us to type any network, thus justifying the name "wild". We start by defining the map gt which associates a pair, made of a network and a list of participants, with a global type. We denote by $\epsilon$ the empty list, by - the concatenation of lists and by $\preceq$ the lexicographic order on labels. We use $\pi$ to range over lists of participants and write part $(\pi)$ for the set of participants occurring in $\pi$. Essentially $\operatorname{gt}(\mathbb{N}, \pi)$ is the global type whose first communication is done by the first participant $\mathrm{p}$ of $\pi$ having a process different from $\mathbf{0}$. If $\mathrm{p}$ does an output, then the global type is an output with the same set of labels. If $p$ does an input, then the global type is an input with only one label. For inputs the global type could have as set of labels any subset of the set of labels in the process; our choice produces simpler global types, which make examples more readable. 
Definition 3.5. Let $\mathbb{N}$ be a network and $\pi$ be a list of participants. The global type gt $(\mathbb{N}, \pi)$ associated with $\mathbb{N}$ and $\pi$ is corecursively defined by the following equations:

$$
\begin{aligned}
& \operatorname{gt}(\mathbb{N}, \epsilon)=\text { End } \\
& \operatorname{gt}(\mathbb{N}, \mathrm{p} \cdot \pi)= \begin{cases}\operatorname{gt}\left(\mathbb{N}^{\prime}, \pi\right) & \text { if } \mathbb{N} \equiv \mathrm{p} \llbracket \mathbf{0} \rrbracket \| \mathbb{N}^{\prime} \\
\mathrm{pq} !\left\{\lambda_{i} ; \operatorname{gt}\left(\mathrm{p} \llbracket P_{i} \rrbracket \| \mathbb{N}^{\prime}, \pi \cdot \mathrm{p}\right)\right\}_{i \in I} & \text { if } \mathbb{N} \equiv \mathrm{p} \llbracket \mathrm{q} !\left\{\lambda_{i} ; P_{i}\right\}_{i \in I} \rrbracket \| \mathbb{N}^{\prime} \\
\mathrm{qp} ? \lambda_{k} ; \operatorname{gt}\left(\mathrm{p} \llbracket P_{k} \rrbracket \| \mathbb{N}^{\prime}, \pi \cdot \mathrm{p}\right) & \text { if } \mathbb{N} \equiv \mathrm{p} \llbracket \mathrm{q} ?\left\{\lambda_{i} ; P_{i}\right\}_{i \in I} \rrbracket \| \mathbb{N}^{\prime} \\
& \text { and } k \in I \text { and } \lambda_{k} \preceq \lambda_{i} \forall i \in I\end{cases}
\end{aligned}
$$

Notice that $\operatorname{gt}(\mathbb{N}, \pi)$ is well defined as equations are productive, i.e., they always eventually unfold a constructor. ${ }^{1}$

Example 3.6. Let $\mathbb{N}$ be the hospital network, i.e. $\mathbb{N} \equiv \mathrm{p} \llbracket P \rrbracket \| \mathrm{s} \llbracket S \rrbracket$, where $P=\mathrm{s} ! n d ; P_{1}$, $P_{1}=\mathrm{s} ?\{o k ; P, k o ; \mathrm{s} ! p r ; P\}, S=\mathrm{p} ?\left\{n d ; S_{1}, p r ; S_{1}\right\}, S_{1}=\mathrm{p} !\{o k ; S, k o ; S\}$. Starting from the participant list $\mathrm{p} \cdot \mathrm{s}$ and letting $\mathbb{N}^{\prime}=\mathrm{p} \llbracket P_{1} \rrbracket \| \mathrm{s} \llbracket S \rrbracket$ we get

$$
\begin{aligned}
& \operatorname{gt}(\mathbb{N}, \mathrm{p} \cdot \mathrm{s})=\mathrm{ps} ! n d ; \mathrm{ps} ? n d ; \mathrm{sp} ? k o ; \mathrm{sp} !\{o k ; \mathrm{ps} ! p r ; \operatorname{gt}(\mathbb{N}, \mathrm{s} \cdot \mathrm{p}), k o ; \mathrm{ps} ! p r ; \operatorname{gt}(\mathbb{N}, \mathrm{s} \cdot \mathrm{p})\} \\
& \operatorname{gt}(\mathbb{N}, \mathrm{s} \cdot \mathrm{p})=\mathrm{ps} ? n d ; \mathrm{ps} ! n d ; \mathrm{sp} !\left\{o k ; \operatorname{gt}\left(\mathbb{N}^{\prime}, \mathrm{p} \cdot \mathrm{s}\right), k o ; \operatorname{gt}\left(\mathbb{N}^{\prime}, \mathrm{p} \cdot \mathrm{s}\right)\right\} \\
& \operatorname{gt}\left(\mathbb{N}^{\prime}, \mathrm{p} \cdot \mathrm{s}\right)=\mathrm{sp} ? k o ; \mathrm{ps} ? n d ; \mathrm{ps} ! p r ; \mathrm{sp} !\{o k ; \operatorname{gt}(\mathbb{N}, \mathrm{p} \cdot \mathrm{s}), k o ; \operatorname{gt}(\mathbb{N}, \mathrm{p} \cdot \mathrm{s})\}
\end{aligned}
$$

Starting from the participant list $\pi=\mathrm{p} \cdot \mathrm{s} \cdot \mathrm{p} \cdot \mathrm{p} \cdot \mathrm{s}$ we get

$$
\operatorname{gt}(\mathbb{N}, \pi)=\mathrm{ps} ! n d ; \mathrm{ps} ? n d ; \mathrm{sp} ? k o ; \mathrm{p} s ! p r ; \mathrm{sp} !\{o k ; \operatorname{gt}(\mathbb{N}, \pi), k o ; \operatorname{gt}(\mathbb{N}, \pi)\}
$$

These examples show that we can obtain different global types for the same network starting from different lists of participants, i.e. we can get $\operatorname{gt}(\mathbb{N}, \pi) \neq \operatorname{gt}\left(\mathbb{N}, \pi^{\prime}\right)$ when $\pi \neq \pi^{\prime}$. Notice that there is no $\pi$ such that $\mathbb{N}$ is the network and $\operatorname{gt}(\mathbb{N}, \pi)$ is the global type of Figure 4 , since in the two branches of the derivation $\mathcal{D}$ the participants $\mathrm{p}$ and $\mathrm{s}$ alternate in different ways.

Since processes are regular terms, they have finite sets of subterms. Therefore given a network $\mathbb{N}$ the set of networks $\mathbb{N}^{\prime}$ such that, if $\mathrm{p} \llbracket P^{\prime} \rrbracket \in \mathbb{N}^{\prime}$, then $\mathrm{p} \llbracket P \rrbracket \in \mathbb{N}$ and $P^{\prime}$ is a subterm of $P$ is finite. Moreover the number of rotations of a list is the length of the list. Therefore $\operatorname{gt}(\mathbb{N}, \pi)$ is a regular term, since it is generated by a finite number of equations.

The following lemma shows why we use the list $\pi$ to build the global type $\operatorname{gt}(\mathbb{N}, \pi)$. Namely, this lemma ensures that, when players $(\mathbb{N}) \subseteq \operatorname{part}(\pi)$, each player of the network $\mathbb{N}$ is also a player of $\mathrm{gt}(\mathbb{N}, \pi)$.

Lemma 3.7. If players $(\mathbb{N}) \subseteq \operatorname{part}(\pi)$, then players $(\mathbb{N})=\operatorname{players}(\operatorname{gt}(\mathbb{N}, \pi))$.

Proof. The inclusion players $(\mathrm{gt}(\mathbb{N}, \pi)) \subseteq$ players $(\mathbb{N})$ easily follows from Definition 3.5. In fact, only a player of $\mathbb{N}$ which occurs as first element of $\pi$ becomes a player of $\operatorname{gt}(\mathbb{N}, \pi)$ and then gt is applied to networks $\mathbb{N}^{\prime}$ such that players $\left(\mathbb{N}^{\prime}\right) \subseteq$ players $(\mathbb{N})$.

To show the reverse implication, i.e. players $(\mathbb{N}) \subseteq$ players $(g t(\mathbb{N}, \pi))$, we define the position of participant $\mathrm{p}$ in the list $\pi$, notation $\operatorname{pos}(\pi, \mathrm{p})$, by:

$$
\operatorname{pos}\left(\mathrm{q} \cdot \pi^{\prime}, \mathrm{p}\right)= \begin{cases}1 & \text { if } \mathrm{p}=\mathrm{q} \\ 1+\operatorname{pos}\left(\pi^{\prime}, \mathrm{p}\right) & \text { otherwise }\end{cases}
$$

\footnotetext{
${ }^{1}$ This can be easily checked as in the only non-productive clause (the first alternative in the second equation) the list of participants decreases.
} 
Let $\mathrm{p}$ be a player of $\mathbb{N}$. The condition players $(\mathbb{N}) \subseteq \operatorname{part}(\pi)$ implies $n=\operatorname{pos}(\pi, \mathrm{p}) \geq 1$. We prove by induction on $n$ that $\mathrm{p} \in \operatorname{players}(\mathrm{gt}(\mathbb{N}, \pi))$. If $n=1$, then $\pi=\mathrm{p} \cdot \pi^{\prime}$ and, since $\mathrm{p}$ is a player of $\mathbb{N}$, we have $\mathbb{N} \equiv \mathrm{p} \llbracket \mathbf{q} \dagger\left\{\lambda_{i} ; P_{i}\right\}_{i \in I} \rrbracket \| \mathbb{N}^{\prime}$. Therefore gt $(\mathbb{N}, \pi)$ is either $\mathrm{pq} !\left\{\lambda_{i} ; \mathrm{G}_{i}\right\}_{i \in I}$ or q p? $\lambda_{k} ; \mathrm{G}_{k}$, where $k \in I$ and $\lambda_{k} \preceq \lambda_{i}$ for all $i \in I$. Hence $\mathrm{p} \in \operatorname{players}(\operatorname{gt}(\mathbb{N}, \pi))$. If $n>1$, then $\pi=\mathrm{q} \cdot \pi^{\prime}$ with $\mathrm{p} \neq \mathrm{q}$ and $\operatorname{pos}(\pi, \mathrm{p})=1+\operatorname{pos}\left(\pi^{\prime}, \mathrm{p}\right)$. We distinguish two cases.

- If $\mathrm{q} \notin$ players $(\mathbb{N})$, that is, $\mathbb{N} \equiv \mathrm{q} \llbracket \mathbf{0} \rrbracket \| \mathbb{N}^{\prime}$, then $\operatorname{gt}(\mathbb{N}, \pi)=\operatorname{gt}\left(\mathbb{N}^{\prime}, \pi^{\prime}\right)$. Since $\operatorname{pos}\left(\pi^{\prime}, \mathrm{p}\right)=$ $n-1$, by induction hypothesis, we get $\mathrm{p} \in \operatorname{players}\left(\operatorname{gt}\left(\mathbb{N}^{\prime}, \pi^{\prime}\right)\right)=\operatorname{players}(\operatorname{gt}(\mathbb{N}, \pi))$, as needed. - Otherwise, $\mathbb{N} \equiv \mathrm{q} \llbracket \mathrm{r} \dagger\left\{\lambda_{i} ; Q_{i}\right\}_{i \in I} \rrbracket \| \mathbb{N}^{\prime}$ and so gt $(\mathbb{N}, \pi)$ is either $\mathrm{q} r !\left\{\lambda_{i} ; \mathrm{G}_{i}\right\}_{i \in I}$ or $\mathrm{rq}$ ? $\lambda_{k} ; \mathrm{G}_{k}$, with $k \in I$ and $\lambda_{k} \preceq \lambda_{i}$ for all $i \in I$, where $\mathrm{G}_{i}=\operatorname{gt}\left(\mathrm{q} \llbracket Q_{i} \rrbracket \| \mathbb{N}^{\prime}, \pi^{\prime} \cdot \mathbf{q}\right)$ for all $i \in I$. Note that, by definition, we have $\operatorname{pos}\left(\pi^{\prime} \cdot \mathrm{q}, \mathrm{p}\right)=\operatorname{pos}\left(\pi^{\prime}, \mathrm{p}\right)=n-1$, then by induction hypothesis, we get $\mathrm{p} \in \operatorname{players}\left(\mathrm{G}_{i}\right)$ for all $i \in I$, hence $\mathrm{p} \in \operatorname{players}(\mathrm{gt}(\mathbb{N}, \mathrm{p}))$.

Theorem 3.8. Let $\mathbb{N}=\mathrm{p}_{1} \llbracket P_{1} \rrbracket\|\cdots\| \mathrm{p}_{n} \llbracket P_{n} \rrbracket$, then $\operatorname{gt}\left(\mathbb{N}, \mathrm{p}_{1} \cdots \mathrm{p}_{n}\right) \vdash \mathbb{N}$.

Proof. The proof is by coinduction, hence we show that the set

$$
\mathcal{G N}=\{(\mathrm{G}, \mathbb{N}) \mid \mathrm{G}=\operatorname{gt}(\mathbb{N}, \pi) \text { for some } \pi \text { such that players }(\mathbb{N}) \subseteq \operatorname{part}(\pi)\}
$$

is consistent with respect to the rules defining the typing judgement $G \vdash \mathbb{N}$, i.e., any $(G, \mathbb{N}) \in \mathcal{G N}$ is the consequence of one of the typing rules whose premises are again in $\mathcal{G N}$. Let $(G, \mathbb{N}) \in \mathcal{G N}$ and split cases on the shape of $G$.

- If $\mathrm{G}=$ End, then End $=\operatorname{gt}(\mathbb{N}, \pi)$ for some $\pi$ such that players $(\mathbb{N}) \subseteq \operatorname{part}(\pi)$. By Lemma 3.7 players $(\mathbb{N})=$ players $($ End $)$, which implies $\mathbb{N} \equiv \mathrm{p} \llbracket \mathbf{0} \rrbracket$. Rule [END] derives End $\vdash \mathrm{p} \llbracket \mathbf{0} \rrbracket$ and has no premises.

- If $\mathrm{G}=\mathrm{pq} !\left\{\lambda_{i} ; \mathrm{G}_{i}\right\}_{i \in I}$, then, by definition of $\mathcal{G N}$, we have pq! $\left\{\lambda_{i} ; \mathrm{G}_{i}\right\}_{i \in I}=\operatorname{gt}(\mathbb{N}, \pi)$ for some $\pi$ such that players $(\mathbb{N}) \subseteq \operatorname{part}(\pi)$. By Definition $3.5 \operatorname{gt}(\mathbb{N}, \pi)=\mathrm{pq} !\left\{\lambda_{i} ; \mathrm{G}_{i}\right\}_{i \in I}$ implies $\pi=\pi^{\prime} \cdot \mathrm{p} \cdot \pi^{\prime \prime}$ with part $\left(\pi^{\prime}\right) \cap \operatorname{players}(\mathbb{N})=\emptyset$ and $\mathbb{N} \equiv \mathrm{p} \llbracket \mathrm{q} !\left\{\lambda_{i} ; P_{i}\right\}_{i \in I} \rrbracket \| \mathbb{N}^{\prime}$ and $\mathrm{G}_{i}=\operatorname{gt}\left(\mathrm{p} \llbracket P_{i} \rrbracket \| \mathbb{N}^{\prime}, \pi^{\prime \prime} \cdot \mathrm{p}\right)$ for all $i \in I$. Therefore $\left(\mathrm{G}_{i}, \mathrm{p} \llbracket P_{i} \rrbracket \| \mathbb{N}^{\prime}\right) \in \mathcal{G} \mathcal{N}$ for all $i \in I$, since $\operatorname{part}\left(\mathrm{p} \cdot \pi^{\prime \prime}\right)=\operatorname{part}\left(\pi^{\prime \prime} \cdot \mathrm{p}\right)$. Moreover, from Lemma 3.7, players $\left(\mathrm{G}_{i}\right)=\operatorname{players}\left(\mathrm{p} \llbracket P_{i} \rrbracket \| \mathbb{N}^{\prime}\right)$ for all $i \in I$. So players $\left(\mathrm{G}_{i}\right) \backslash\{\mathrm{p}\}=\operatorname{players}\left(\mathbb{N}^{\prime}\right)$ and Rule [OUT] can be used to derive $\mathrm{pq} !\left\{\lambda_{i} ; \mathrm{G}_{i}\right\}_{i \in I} \vdash \mathbb{N}$ from premises in $\mathcal{G N}$.

- If $\mathrm{G}$ is an input type, then, by definition of $\mathcal{G N}$, we get $\mathrm{G}=\mathrm{q} p$ ? $\lambda ; \mathrm{G}^{\prime}$ for some $\lambda$ and $\mathrm{G}^{\prime}$ and $\mathrm{qp} ? \lambda ; \mathrm{G}^{\prime}=\operatorname{gt}(\mathbb{N}, \pi)$ for some $\pi$ such that players $(\mathbb{N}) \subseteq \operatorname{part}(\pi)$. By Definition $3.5 \operatorname{gt}(\mathbb{N}, \pi)=$ $\mathrm{q} \mathrm{p} ? \lambda ; \mathrm{G}^{\prime}$ implies $\pi=\pi^{\prime} \cdot \mathrm{p} \cdot \pi^{\prime \prime}$ with part $\left(\pi^{\prime}\right) \cap$ players $(\mathbb{N})=\emptyset$ and $\mathbb{N} \equiv \mathrm{p} \llbracket \mathrm{q} ?\left\{\lambda_{i} ; P_{i}\right\}_{i \in I} \rrbracket \| \mathbb{N}^{\prime}$ and $\lambda=\lambda_{k}$ and $\mathrm{G}^{\prime}=\mathrm{G}_{k}=\operatorname{gt}\left(\mathrm{p} \llbracket P_{k} \rrbracket \| \mathbb{N}^{\prime}, \pi^{\prime \prime} \cdot \mathrm{p}\right)$ for some $k \in I$ such that $\lambda_{k} \preceq \lambda_{i}$ for all $i \in I$. Therefore $\left(\mathrm{G}^{\prime}, \mathrm{p} \llbracket P_{k} \rrbracket \| \mathbb{N}^{\prime}\right) \in \mathcal{G N}$, since $\operatorname{part}\left(\mathrm{p} \cdot \pi^{\prime \prime}\right)=\operatorname{part}\left(\pi^{\prime \prime} \cdot \mathrm{p}\right)$. Moreover, from Lemma 3.7, players $\left(\mathrm{G}^{\prime}\right)=$ players $\left(\mathrm{p} \llbracket P_{k} \rrbracket \| \mathbb{N}^{\prime}\right)$. So players $\left(\mathrm{G}^{\prime}\right) \backslash\{\mathrm{p}\}=$ players $\left(\mathbb{N}^{\prime}\right)$ and Rule [IN] can be used to derive q $\mathrm{p}$ ? $\lambda ; \mathrm{G}^{\prime} \vdash \mathbb{N}$ from premises in $\mathcal{G N}$.

Then, the thesis follows, since $\left(\operatorname{gt}\left(\mathbb{N}, \mathrm{p}_{1} \cdots \mathrm{p}_{n}\right), \mathbb{N}\right) \in \mathcal{G N}$ for $\mathbb{N} \equiv \mathrm{p}_{1} \llbracket P_{1} \rrbracket\|\cdots\| \mathrm{p}_{n} \llbracket P_{n} \rrbracket$.

Global types provide an overall description of asynchronous multiparty protocols and their semantics is specified by an LTS on types in parallel with queues, dubbed type configurations. Reduction rules are reported in Figure 5. The first two rules reduce outputs and inputs at top level in the standard way. The remaining two rules reduce inside output and input choices. These rules are needed to enable interleaving between independent communications despite the sequential structure of global types. For example, we want to allow $\mathrm{pq} ! \lambda ; \mathrm{rs} ! \lambda^{\prime}\left\|\emptyset \stackrel{\mathrm{r} s ! \lambda^{\prime}}{\longrightarrow} \mathrm{pq} ! \lambda\right\|\left\langle\mathrm{r}, \lambda^{\prime}, \mathrm{s}\right\rangle$ when $\mathrm{p} \neq \mathrm{r}$, because, intuitively, actions performed by different players should be independent. This justifies the conditions $\mathbf{p} \neq \operatorname{play}(\beta)$ and $\mathbf{q} \neq \operatorname{play}(\beta)$ in Rules [InSIDE-OUT] and [InSIDE-IN], respectively. The requirements on the 


$$
\begin{aligned}
& \text { [Top-Out] } \underset{\mathrm{pq} !\left\{\lambda_{i} ; \mathrm{G}_{i}\right\}_{i \in I}\left\|\mathcal{M} \stackrel{\mathrm{pq} ! \lambda_{h}}{\longrightarrow} \mathrm{G}_{h}\right\| \mathcal{M} \cdot\left\langle\mathrm{p}, \lambda_{h}, \mathrm{q}\right\rangle}{h \in I} \\
& \text { [Top-In] } \underset{\mathrm{pq} ?\left\{\lambda_{i} ; \mathrm{G}_{i}\right\}_{i \in I}\left\|\left\langle\mathrm{p}, \lambda_{h}, \mathrm{q}\right\rangle \cdot \mathcal{M} \stackrel{\mathrm{pq} ? \lambda_{h}}{\longrightarrow} \mathrm{G}_{h}\right\| \mathcal{M}}{h \in I} \\
& {[\text { Inside-Out }] \frac{\mathrm{G}_{i}\left\|\mathcal{M} \cdot\left\langle\mathrm{p}, \lambda_{i}, \mathrm{q}\right\rangle \stackrel{\beta}{\rightarrow} \mathrm{G}_{i}^{\prime}\right\| \mathcal{M}^{\prime} \cdot\left\langle\mathrm{p}, \lambda_{i}, \mathrm{q}\right\rangle \quad \forall i \in I}{\mathrm{pq} !\left\{\lambda_{i} ; \mathrm{G}_{i}\right\}_{i \in I}\left\|\mathcal{M} \stackrel{\beta}{\rightarrow} \mathrm{pq} !\left\{\lambda_{i} ; \mathrm{G}_{i}^{\prime}\right\}_{i \in I}\right\| \mathcal{M}^{\prime}} \quad \mathrm{p} \neq \operatorname{play}(\beta)} \\
& {\left[\text { Inside-IN] } \frac{\mathrm{G}_{i}\left\|\mathcal{M} \stackrel{\beta}{\rightarrow} \mathrm{G}_{i}^{\prime}\right\| \mathcal{M}^{\prime} \quad \forall i \in I}{\mathrm{pq} ?\left\{\lambda_{i} ; \mathrm{G}_{i}\right\}_{i \in I}\left\|\left\langle\mathrm{p}, \lambda_{h}, \mathrm{q}\right\rangle \cdot \mathcal{M} \stackrel{\beta}{\rightarrow} \mathrm{pq} ?\left\{\lambda_{i} ; \mathrm{G}_{i}^{\prime}\right\}_{i \in I}\right\|\left\langle\mathrm{p}, \lambda_{h}, \mathrm{q}\right\rangle \cdot \mathcal{M}^{\prime}} \quad \mathrm{q} \neq \operatorname{play}(\beta) h \in I\right.}
\end{aligned}
$$

Figure 5: LTS for type configurations.

shapes of queues in these rules are more interesting. They enforce that inputs and outputs on the same channel, namely, the same ordered pair of participants, happen in the order prescribed by the global type, that is, an input cannot consume a message produced by a subsequent output. Indeed, if we would take the following version of Rule [INSIDE-IN]

$$
\frac{\mathrm{G}_{i}\left\|\mathcal{M} \stackrel{\beta}{\rightarrow} \mathrm{G}_{i}^{\prime}\right\| \mathcal{M}^{\prime} \quad \forall i \in I}{\mathrm{pq} ?\left\{\lambda_{i} ; \mathrm{G}_{i}\right\}_{i \in I}\left\|\mathcal{M} \stackrel{\beta}{\rightarrow} \mathrm{pq} ?\left\{\lambda_{i} ; \mathrm{G}_{i}^{\prime}\right\}_{i \in I}\right\| \mathcal{M}^{\prime}} \quad \mathrm{q} \neq \operatorname{play}(\beta)
$$

we would get $\mathrm{pq} ? \lambda ; \mathrm{pq} ! \lambda\|\emptyset \stackrel{\mathrm{pq} ! \lambda}{\longrightarrow} \mathrm{pq} ? \lambda\|\langle\mathrm{p}, \lambda, \mathrm{q}\rangle \stackrel{\mathrm{pq} ? \lambda}{\longrightarrow}$ End $\| \emptyset$. This reduction breaks the order on the channel pq prescribed by the global type $\mathrm{pq} ? \lambda ; \mathrm{pq} ! \lambda$, which is to do first an input and then the corresponding output. In our LTS p q? $\lambda ; p q ! \lambda \| \emptyset$ is instead stuck, as it should. In fact, the shape of the queue in Rule [INSIDE-IN] ensures that $\beta$ is not the matching output for any input in the choice. Similarly, the shapes of queues in Rule [Inside-OUT] ensures that $\beta$ is not the matching input for any output in the choice.

A last remark is that the LTS rules are inductive. Therefore, the premises of Rules [Inside-Out] and [Inside-In] oblige play $(\beta)$ to occur in all $\mathrm{G}_{i}$ for $i \in I$. This condition is in line with the boundedness of global types, see Definition 4.2.

We are now ready to state and prove Session Fidelity, showing that a session can correctly perform the protocol described by the global type derivable for its network. The reverse property, i.e. that the sessions can only do inputs and outputs allowed by the global types of their networks, will be proved for "tamed" global types in the Subject Reduction Theorem, Theorem 4.7.

Theorem 3.9 (Session Fidelity). If $\mathrm{G} \vdash \mathbb{N}$ and $\mathrm{G}\left\|\mathcal{M} \stackrel{\beta}{\rightarrow} \mathrm{G}^{\prime}\right\| \mathcal{M}^{\prime}$, then $\mathbb{N}\left\|\mathcal{M} \stackrel{\beta}{\rightarrow} \mathbb{N}^{\prime}\right\| \mathcal{M}^{\prime}$ and $\mathrm{G}^{\prime} \vdash \mathbb{N}^{\prime}$.

Proof. The proof is by induction on the reduction rules.

[Top-IN]: Then $\mathrm{G}=\mathrm{pq} ?\left\{\lambda_{i} ; \mathrm{G}_{i}\right\}_{i \in I}$ and $\mathcal{M} \equiv\left\langle\mathrm{p}, \lambda_{h}, \mathrm{q}\right\rangle \cdot \mathcal{M}^{\prime}$ and $\beta=\mathrm{pq} ? \lambda_{h}$ and $\mathrm{G}^{\prime}=\mathrm{G}_{h}$ with $h \in I$. Since $\mathbf{G} \vdash \mathbb{N}$ must be derived using rule $[\mathrm{IN}]$ we get $\mathbb{N} \equiv \mathrm{p} \llbracket \mathrm{q} ?\left\{\lambda_{j} ; P_{j}\right\}_{j \in J} \rrbracket \|$ $\mathbb{N}_{0}$ with $I \subseteq J$ and $\mathrm{G}_{i} \vdash \mathrm{p} \llbracket P_{i} \rrbracket \| \mathbb{N}_{0}$ for all $i \in I$. We conclude $\mathbb{N}\left\|\mathcal{M} \stackrel{\beta}{\rightarrow} \mathrm{p} \llbracket P_{h} \rrbracket\right\|$ $\mathbb{N}_{0} \| \mathcal{M}^{\prime}$ by Rule $[\mathrm{RCV}]$ and $\mathrm{G}_{h} \vdash \mathrm{p} \llbracket P_{h} \rrbracket \| \mathbb{N}_{0}$. 
[INSIDE-OUT]: Then $\mathrm{G}=\mathrm{pq} !\left\{\lambda_{i} ; \mathrm{G}_{i}\right\}_{i \in I}$ and $\mathrm{G}^{\prime}=\mathrm{pq} !\left\{\lambda_{i} ; \mathrm{G}_{i}^{\prime}\right\}_{i \in I}$ and $\mathrm{G}_{i} \| \mathcal{M} \cdot\left\langle\mathrm{p}, \lambda_{i}, \mathrm{q}\right\rangle \stackrel{\beta}{\rightarrow}$ $\mathrm{G}_{i}^{\prime} \| \mathcal{M}^{\prime} \cdot\left\langle\mathrm{p}, \lambda_{i}, \mathrm{q}\right\rangle$ for all $i \in I$ and $\mathrm{p} \neq \operatorname{play}(\beta)$. Since $\mathrm{G} \vdash \mathbb{N}$ must be derived using rule [OUT] we get $\mathbb{N} \equiv \mathrm{p} \llbracket \mathrm{q} !\left\{\lambda_{i} ; P_{i}\right\}_{i \in I} \rrbracket \| \mathbb{N}_{0}$ and $\mathrm{G}_{i} \vdash \mathrm{p} \llbracket P_{i} \rrbracket \| \mathbb{N}_{0}$ for all $i \in I$. By induction $\mathrm{p} \llbracket P_{i} \rrbracket\left\|\mathbb{N}_{0}\right\| \mathcal{M} \cdot\left\langle\mathrm{p}, \lambda_{i}, \mathrm{q}\right\rangle \stackrel{\beta}{\rightarrow} \mathbb{N}_{i} \| \mathcal{M}^{\prime} \cdot\left\langle\mathrm{p}, \lambda_{i}, \mathrm{q}\right\rangle$ and $\mathrm{G}_{i}^{\prime} \vdash \mathbb{N}_{i}$ for all $i \in I$. The condition $\mathrm{p} \neq \operatorname{play}(\beta)$ ensures that these reductions do not modify the processes of participant p, i.e. $\mathbb{N}_{i} \equiv \mathrm{p} \llbracket P_{i} \rrbracket \| \mathbb{N}_{0}^{\prime}$ for all $i \in I$ and some $\mathbb{N}_{0}^{\prime}$. Moreover these reductions do not depend on the messages $\left\langle\mathrm{p}, \lambda_{i}\right.$, q $\rangle$, which are in the queue before and after these reductions. We get $\mathbb{N}\left\|\mathcal{M} \stackrel{\beta}{\rightarrow} \mathrm{p} \llbracket \mathrm{q} !\left\{\lambda_{i} ; P_{i}\right\}_{i \in I} \rrbracket\right\| \mathbb{N}_{0}^{\prime} \| \mathcal{M}^{\prime}$. By Lemma 3.2 $\mathrm{G}_{i}^{\prime} \vdash \mathrm{p} \llbracket P_{i} \rrbracket \| \mathbb{N}_{0}^{\prime}$ implies players $\left(\mathrm{G}_{i}^{\prime}\right) \backslash\{\mathrm{p}\}=\operatorname{players}\left(\mathbb{N}_{0}^{\prime}\right)$ for all $i \in I$. Then we can derive $\mathrm{G}^{\prime} \vdash \mathrm{p} \llbracket \mathrm{q} !\left\{\lambda_{i} ; P_{i}\right\}_{i \in I} \rrbracket \| \mathbb{N}_{0}^{\prime}$ using Rule [OUT].

The proofs for the remaining rules are similar.

We extend the properties of multiparty sessions to type configurations. Let us define the lockstep transition relation $\mathrm{G}\left\|\mathcal{M} \stackrel{\Delta}{\Rightarrow} \mathrm{G}^{\prime}\right\| \mathcal{M}^{\prime}$ by:

$\mathrm{G}\left\|\mathcal{M} \triangleq \mathrm{G}^{\prime}\right\| \mathcal{M}^{\prime}$ if $\Delta=\left\{\beta_{1}, \ldots, \beta_{n}\right\}$ is a maximal coherent set for $\mathrm{G} \| \mathcal{M}$ and

$$
\mathrm{G}\left\|\mathcal{M} \stackrel{\beta_{1}}{\longrightarrow} \cdots \stackrel{\beta_{n}}{\longrightarrow} \mathrm{G}^{\prime}\right\| \mathcal{M}^{\prime}
$$

where $\Delta$ is coherent for $\mathrm{G} \| \mathcal{M}$ if

(1) for all $\beta_{1}, \beta_{2} \in \Delta$, play $\left(\beta_{1}\right)=\operatorname{play}\left(\beta_{2}\right)$ implies $\beta_{1}=\beta_{2}$, and

(2) for all $\beta \in \Delta, \mathrm{G} \| \mathcal{M} \stackrel{\beta}{\rightarrow}$.

A type configuration $\mathrm{G} \| \mathcal{M}$ is deadlocked if $\mathrm{G} \neq$ End and there is no $\beta$ such that $\mathrm{G} \| \mathcal{M} \stackrel{\beta}{\rightarrow}$.

Definition 3.10 (Input-enabling type configuration). A type configuration $\mathrm{G} \| \mathcal{M}$ is inputenabling if $\mathrm{p} \in$ players $(\mathrm{G})$ implies that, for all complete

$$
\gamma=\left\{\mathrm{G}_{k}\left\|\mathcal{M}_{k} \stackrel{\Delta_{k}}{\underset{k}{\rightleftarrows}} \mathrm{G}_{k+1}\right\| \mathcal{M}_{k+1}\right\}_{k<x}
$$

with $\mathrm{G}_{0}\left\|\mathcal{M}_{0}=\mathrm{G}\right\| \mathcal{M}$, there exists $h<x$ such that $\beta \in \Delta_{h}$ with play $(\beta)=\mathrm{p}$.

This definition ensures that no player is stuck, and this coincides with input enabling, since outputs can always be done.

Definition 3.11 (Queue-consuming type configuration). A type configuration $\mathrm{G} \| \mathcal{M}$ is queue-consuming if $\mathcal{M} \equiv\langle\mathrm{p}, \lambda, \mathrm{q}\rangle \cdot \mathcal{M}^{\prime}$ implies that, for all complete

$$
\gamma=\left\{\mathrm{G}_{k}\left\|\mathcal{M}_{k} \stackrel{\Delta_{k}}{\underset{k}{\longrightarrow}} \mathrm{G}_{k+1}\right\| \mathcal{M}_{k+1}\right\}_{k<x}
$$

with $\mathrm{G}_{0}\left\|\mathcal{M}_{0}=\mathrm{G}\right\| \mathcal{M}$, there exists $h<x$ such that $\mathrm{pq} ? \lambda \in \Delta_{h}$.

3.2. Type Inference. In this subsection, we will describe an algorithm to infer global types from networks, proving its soundness and completeness with respect to the typing system. That is, the algorithm applied to a network $\mathbb{N}$ enumerats all and only those global types which can be derived for $\mathbb{N}$. Note that, since a network may have more than one global type, to be complete, the algorithm needs to be non-deterministic. 


$$
\begin{aligned}
& {[\text { I-END }] \overline{\mathcal{S} \vdash(\mathrm{p} \llbracket \mathbf{0} \rrbracket, X) \Rightarrow\{X \mp \text { End }\}}} \\
& {[\text { I-CYCLE }] \overline{\mathcal{S},(\mathbb{N}, Y) \vdash(\mathbb{N}, X) \Rightarrow\{X \mp Y\}}}
\end{aligned}
$$

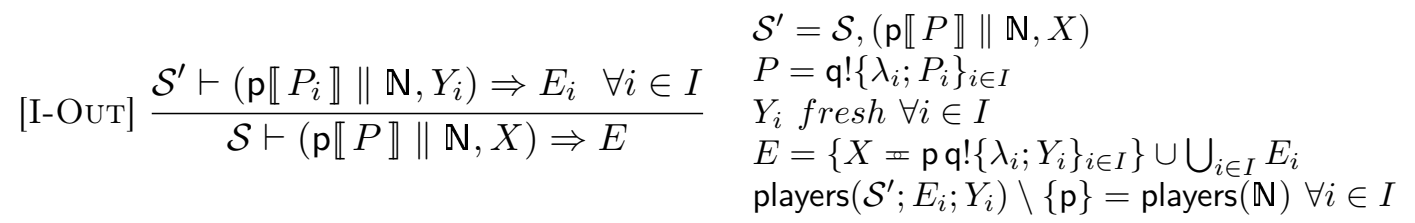

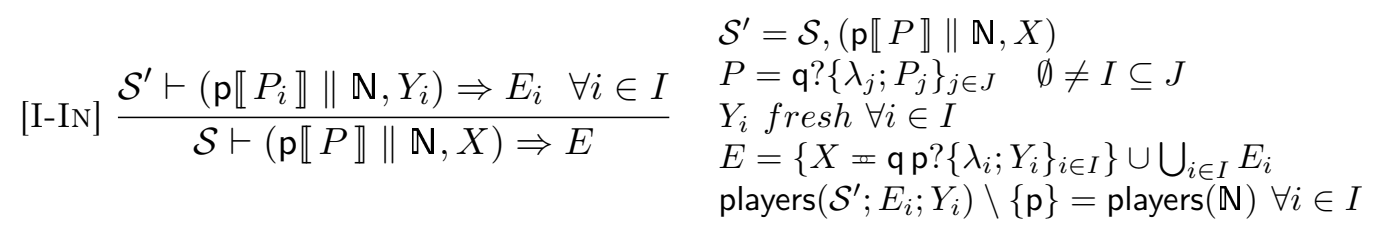

Figure 6: Rules of the inference algorithm.

The first step towards defining an algorithm is the introduction of a finite representation for global types. ${ }^{2}$ Since global types are regular terms, from results in [Cou83, AMV06], they can be represented as finite systems of regular syntactic equations formally defined below. First, a global type pattern is a finite term generated by the following grammar:

$$
\mathbb{G}::=\text { End }\left|\mathrm{pq} !\left\{\lambda_{i} ; \mathbb{G}_{i}\right\}_{i \in I}\right| \mathrm{pq} ?\left\{\lambda_{i} ; \mathbb{G}_{i}\right\}_{i \in I} \mid X
$$

where $X$ is a variable taken from a countably infinite set. We denote by vars $(\mathbb{G})$ the set of variables occurring in $\mathbb{G}$.

A substitution $\theta$ is a finite partial map from variables to global types. We denote by $\theta+\sigma$ the union of two substitutions such that $\theta(X)=\sigma(X)$, for all $X \in \operatorname{dom}(\theta) \cap \operatorname{dom}(\sigma)$, and by $\mathbb{G} \theta$ the application of $\theta$ to $\mathbb{G}$. We define $\theta \preceq \sigma$ if $\operatorname{dom}(\theta) \subseteq \operatorname{dom}(\sigma)$ and $\theta(X)=\sigma(X)$, for all $X \in \operatorname{dom}(\theta)$. Note that, if vars $(\mathbb{G}) \subseteq \operatorname{dom}(\theta)$, then $\mathbb{G} \theta$ is a global type.

An equation has shape $X=\mathbb{G}$ and a (regular) system of equations $E$ is a finite set of equations such that $X=\mathbb{G}_{1}$ and $X=\mathbb{G}_{2} \in E$ imply $\mathbb{G}_{1}=\mathbb{G}_{2}$. We denote by vars $(E)$ the set $\bigcup\{\operatorname{vars}(\mathbb{G}) \cup\{X\} \mid X=\mathbb{G} \in E\}$ and by $\operatorname{dom}(E)$ the set $\{X \mid X=\mathbb{G} \in E\}$. A solution of a system $E$ is a substitution $\theta$ such that $\operatorname{vars}(E) \subseteq \operatorname{dom}(\theta)$ and, for all $X=\mathbb{G} \in E, \theta(X)=\mathbb{G} \theta$ holds. We denote by $\operatorname{sol}(E)$ the set of all solutions of $E$ and note that $E_{1} \subseteq E_{2}$ implies $\operatorname{sol}\left(E_{2}\right) \subseteq \operatorname{sol}\left(E_{1}\right)$.

The algorithm follows essentially the structure of coSLD resolution of coinductive logic programming [Sim06, SBMG07, SMBG06, AD15], namely, the extension of standard SLD resolution capable to deal with regular terms and coinductive predicates. A goal is a pair $(\mathbb{N}, X)$ of a network $\mathbb{N}$ and a variable $X$. The algorithm takes as input a goal $(\mathbb{N}, X)$ and returns a set of equations $E$ such that the solution for the variable $X$ in $E$ is a global type for the network $\mathbb{N}$. The key idea borrowed from coinductive logic programming is to keep track of already encountered goals to detect cycles, avoiding non-termination.

The inference judgement has the following shape $\mathcal{S} \vdash(\mathbb{N}, X) \Rightarrow E$, where $\mathcal{S}$ is a set of goals, all with different variables which are all different from $X$. Rules defining the inference algorithm are reported in Figure 6. For a terminated network the algorithm returns just one equation $X=$ End (Rule [I-END]). For other networks, the algorithm selects a participant

\footnotetext{
${ }^{2}$ In a similar way we can and should give a finite representation for processes, but we avoid it to reduce noise.
} 
and analyses its process. If the process is an output choice (Rule [I-OUT]), the algorithm continues analysing all branches of the output choice. If the process is an input choice (Rule [I-IN]), the algorithm selects a subset of its branches and continues analysing them. In both cases, subnetworks are analysed adding to the set $\mathcal{S}$ the goal in the conclusion of the rule, to be able to subsequently recognise when a cycle is encountered. After having evaluated subnetwork, the algorithm collects all the resulting equations plus another one for the current variable. Note that variables for goals in the premises are fresh. This is important to ensure that the set of equations $E$ in the conclusion is indeed a regular system of equations (there is at most one equation for each variable). Finally, Rule [I-CYCLE] detects cycles: if the network in the current goal appears also in the set $\mathcal{S}$ the algorithm can stop, returning just one equation unifying the two variables associated with the network.

In Rules [I-OUT] and [I-IN], the side condition players $\left(\mathcal{S}^{\prime} ; E_{i} ; Y_{i}\right) \backslash\{\mathrm{p}\}=\operatorname{players}(\mathbb{N})$ for all $i \in I$ is needed to ensure that the resulting global type associated with the variable $X$ satisfies the conditions on players required by Rules [OUT] and [IN] in Figure 3. The set players $(\mathcal{S} ; E ; \mathbb{G})$ is defined as the set of players of a global type, but with the following additional clause to handle variables:

$$
\operatorname{players}(\mathcal{S} ; E ; X)= \begin{cases}\operatorname{players}(\mathcal{S} ; E ; \mathbb{G}) & \text { if } X \pm \mathbb{G} \in E \\ \operatorname{players}(\mathbb{N}) & \text { if } X \notin \operatorname{dom}(E) \text { and }(\mathbb{N}, X) \in \mathcal{S} \\ \emptyset & \text { otherwise }\end{cases}
$$

Let $E$ be a system of equations and $\mathcal{S}$ a set of goals. A solution $\theta \in \operatorname{sol}(E)$ agrees with $\mathcal{S}$ if $(\mathbb{N}, X) \in \mathcal{S}$ implies players $(\theta(X))=\operatorname{players}(\mathbb{N})$ for all $X \in \operatorname{vars}(E)$. We denote by $\operatorname{sol}_{\mathcal{S}}(E)$ the set of all solutions of $E$ agreeing with $\mathcal{S}$. We say that a system of equations $E$ is guarded if $X=Y$ and $Y=\mathbb{G}$ in $E$ imply that $\mathbb{G}$ is not a variable. Finally, $E$ is $\mathcal{S}$-closed if it is guarded and $\operatorname{dom}(E) \cap \operatorname{vars}(\mathcal{S})=\emptyset$ and $\operatorname{vars}(E) \backslash \operatorname{dom}(E) \subseteq \operatorname{vars}(\mathcal{S})$.

Example 3.12. Figure 7 shows an application of the inference algorithm to the hospital network for getting a set of equations whose solution is the global type derived for this network in Figure 4. We notice that $\mathcal{I}$ and $\mathcal{I}^{\prime}$ in Figure 7 only differ for the names of variables, so they become the same derivation $\mathcal{D}$ in Figure 4 . The set of obtained equation is

$$
\begin{array}{lll}
X=\mathrm{ps} ! n d ; X_{1} & X_{1}=\mathrm{ps} ?\left\{n d ; X_{2}, p r ; X_{2}^{\prime}\right\} & \\
X_{2}=\mathrm{sp} !\left\{o k ; X_{3}, k o ; X_{5}\right\} & X_{3}=\mathrm{sp} ? o k ; X_{4} & X_{4}=X \\
X_{5}=\mathrm{sp} ? k o ; X_{6} & X_{6}=\mathrm{ps} ! p r ; X_{7} & X_{7}=X \\
X_{2}^{\prime}=\mathrm{sp} !\left\{o k ; X_{3}^{\prime}, k o ; X_{5}^{\prime}\right\} & X_{3}^{\prime}=\mathrm{sp} ? o k ; X_{4}^{\prime} & X_{4}^{\prime}=X \\
X_{5}^{\prime}=\mathrm{sp} ? k o ; X_{6}^{\prime} & X_{6}^{\prime}=\mathrm{ps} ! p r ; X_{7}^{\prime} & X_{7}^{\prime}=X
\end{array}
$$

Toward proving properties of the inference algorithm, we check a couple of auxiliary lemmas.

Lemma 3.13. If $\mathcal{S} \vdash(\mathbb{N}, X) \Rightarrow E$, then $E$ is $\mathcal{S}$-closed.

Proof. By a straightforward induction on the derivation of $\mathcal{S} \vdash(\mathbb{N}, X) \Rightarrow E$.

Lemma 3.14. If $E$ is an $\mathcal{S}$-closed system of equations and $\operatorname{vars}(\mathbb{G}) \subseteq \operatorname{vars}(E)$, then $\operatorname{players}(\mathcal{S} ; E ; \mathbb{G})=\operatorname{players}(\mathbb{G} \theta)$ for all $\theta \in \operatorname{sol}_{\mathcal{S}}(E)$.

Proof. To prove the inclusion players $(\mathbb{G} \theta) \subseteq \operatorname{players}(\mathcal{S} ; E ; \mathbb{G})$, let $\mathrm{p} \in \operatorname{players}(\mathbb{G} \theta)$. We show $\mathrm{p} \in \operatorname{players}(\mathcal{S} ; E ; \mathbb{G})$ by induction on the least distance $d$ of a communication with player $\mathrm{p}$ from the root of $\mathbb{G} \theta$. First of all, it is easy to see that there is $\mathbb{G}^{\prime}$ such that players $(\mathcal{S} ; E ; \mathbb{G})=\operatorname{players}\left(\mathcal{S} ; E ; \mathbb{G}^{\prime}\right)$ and $\mathbb{G} \theta=\mathbb{G}^{\prime} \theta$ and either $\mathbb{G}^{\prime}=\mathrm{rs} \dagger\left\{\lambda_{i} ; \mathbb{G}_{i}\right\}_{i \in I}$ or $\mathbb{G}^{\prime}=X$ 


$$
\begin{aligned}
& \frac{\mathcal{I}}{\frac{(\mathrm{p} \llbracket P \rrbracket \| \mathrm{s} \llbracket S \rrbracket, X) \vdash\left(\mathrm{p} \llbracket P_{1} \rrbracket \| \mathrm{s} \llbracket S \rrbracket, X_{1}\right) \Rightarrow E_{1}}{\emptyset \vdash(\mathrm{p} \llbracket P \rrbracket \| \mathrm{s} \llbracket S \rrbracket, X) \Rightarrow E}[\text { I-OUT }]}[\text { I-IN }] \\
& \text { where } \mathcal{I} \text { is } \\
& \begin{array}{ll}
\frac{\mathcal{S}_{1} \vdash\left(\mathrm{p} \llbracket P \rrbracket \| \mathrm{s} \llbracket S \rrbracket, X_{4}\right) \Rightarrow E_{4}}{\mathcal{S} \vdash\left(\mathrm{I}-\mathrm{p} \llbracket P_{1} \rrbracket \| \mathrm{s} \llbracket S \rrbracket, X_{3}\right) \Rightarrow E_{3}}[\mathrm{I}-\mathrm{IN}] & \frac{\mathcal{S}_{3} \vdash\left(\mathrm{p} \llbracket P \rrbracket \| \mathrm{s} \llbracket S \rrbracket, X_{7}\right) \Rightarrow E_{7}}{\text { [I-CYCLE] }} \\
\frac{\mathcal{S}_{2} \vdash\left(\mathrm{p} \llbracket \mathrm{s} ! p r ; P \rrbracket \| \mathrm{s} \llbracket S \rrbracket, X_{6}\right) \Rightarrow E_{6}}{\mathcal{S} \vdash P \rrbracket \| \mathrm{s} \llbracket S \rrbracket, X),\left(\mathrm{p} \llbracket P_{1} \rrbracket\left\|\mathrm{s} \llbracket S \rrbracket, P_{1} \rrbracket\right\| \mathrm{s} \llbracket S \rrbracket, X_{5}\right) \Rightarrow E_{5}}[\mathrm{I} \text {-IN] }
\end{array} \\
& \text { and } \mathcal{I}^{\prime} \text { is }
\end{aligned}
$$

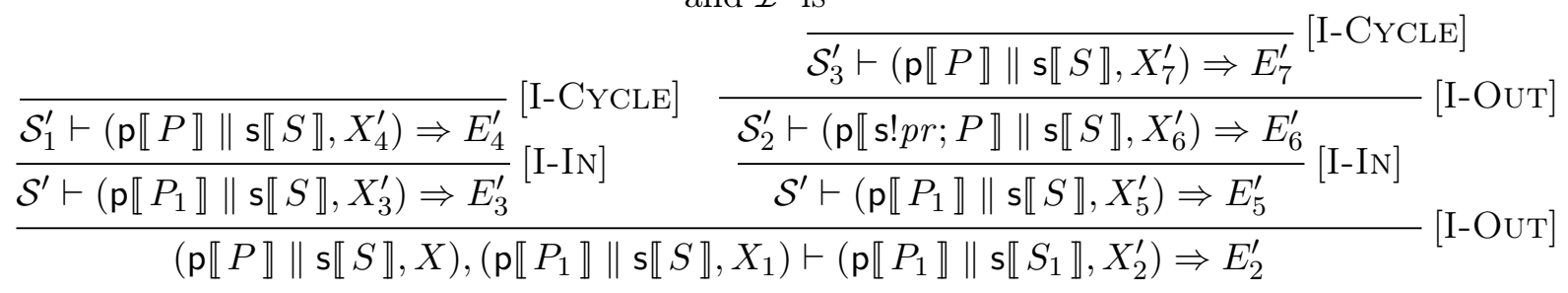

$$
\begin{aligned}
& \text { and } \\
& E=\left\{X=\mathrm{ps} ! n d ; X_{1}\right\} \cup E_{1} \quad E_{1}=\left\{X_{1}=\mathrm{ps} ?\left\{n d ; X_{2}, p r ; X_{2}^{\prime}\right\}\right\} \cup E_{2} \cup E_{2}^{\prime} \\
& E_{2}=\left\{X_{2}=\mathrm{sp} !\left\{o k ; X_{3}, k o ; X_{5}\right\}\right\} \cup E_{3} \cup E_{5} \quad E_{3}=\left\{X_{3}=\mathrm{sp} ? \mathrm{ok} ; X_{4}\right\} \cup E_{4} \\
& E_{4}=\left\{X_{4}=X\right\} \quad E_{5}=\left\{X_{5}=\mathrm{sp} ? k o ; X_{6}\right\} \cup E_{6} \\
& E_{6}=\left\{X_{6}=\mathrm{ps} ! p r ; X_{7}\right\} \cup E_{7} \quad E_{7}=\left\{X_{7} \mp X\right\} \\
& E_{2}^{\prime}=\left\{X_{2}^{\prime}=\mathrm{sp} !\left\{o k ; X_{3}^{\prime}, k o ; X_{5}^{\prime}\right\}\right\} \cup E_{3}^{\prime} \cup E_{5}^{\prime} \quad E_{3}^{\prime}=\left\{X_{3}^{\prime}=\mathrm{sp} ? \circ k ; X_{4}^{\prime}\right\} \cup E_{4}^{\prime} \\
& E_{4}^{\prime}=\left\{X_{4}^{\prime} \equiv X\right\} \quad E_{5}^{\prime}=\left\{X_{5}^{\prime} \equiv \mathrm{sp} ? k o ; X_{6}^{\prime}\right\} \cup E_{6}^{\prime} \\
& E_{6}^{\prime}=\left\{X_{6}^{\prime} \approx \mathrm{ps} ! p r ; X_{7}^{\prime}\right\} \cup E_{7}^{\prime} \quad E_{7}^{\prime}=\left\{X_{7}^{\prime} \mp X\right\} \\
& \mathcal{S}=\left\{(\mathrm{p} \llbracket P \rrbracket \| \mathrm{s} \llbracket S \rrbracket, X),\left(\mathrm{p} \llbracket P_{1} \rrbracket \| \mathrm{s} \llbracket S \rrbracket, X_{1}\right),\left(\mathrm{p} \llbracket P_{1} \rrbracket \| \mathrm{s} \llbracket S_{1} \rrbracket, X_{2}\right)\right\} \\
& \mathcal{S}^{\prime}=\left\{(\mathrm{p} \llbracket P \rrbracket \| \mathrm{s} \llbracket S \rrbracket, X),\left(\mathrm{p} \llbracket P_{1} \rrbracket \| \mathrm{s} \llbracket S \rrbracket, X_{1}\right),\left(\mathrm{p} \llbracket P_{1} \rrbracket \| \mathrm{s} \llbracket S_{1} \rrbracket, X_{2}^{\prime}\right)\right\} \\
& \mathcal{S}_{1}=\mathcal{S} \cup\left\{\left(\mathrm{p} \llbracket P_{1} \rrbracket \| \mathrm{s} \llbracket S \rrbracket, X_{3}\right)\right\} \quad \mathcal{S}_{1}^{\prime}=\mathcal{S}^{\prime} \cup\left\{\left(\mathrm{p} \llbracket P_{1} \rrbracket \| \mathrm{s} \llbracket S \rrbracket, X_{3}^{\prime}\right)\right\} \\
& \mathcal{S}_{2}=\mathcal{S} \cup\left\{\left(\mathrm{p} \llbracket P_{1} \rrbracket \| \mathrm{s} \llbracket S \rrbracket, X_{5}\right)\right\} \quad \mathcal{S}_{2}^{\prime}=\mathcal{S}^{\prime} \cup\left\{\left(\mathrm{p} \llbracket P_{1} \rrbracket \| \mathrm{s} \llbracket S \rrbracket, X_{5}^{\prime}\right)\right\} \\
& \mathcal{S}_{3}=\mathcal{S}_{2} \cup\left\{\left(\mathrm{p} \llbracket \mathrm{s} ! p r ; P \rrbracket \| \mathrm{s} \llbracket S \rrbracket, X_{6}\right)\right\} \quad \mathcal{S}_{3}^{\prime}=\mathcal{S}_{2}^{\prime} \cup\left\{\left(\mathrm{p} \llbracket \mathrm{s} ! p r ; P \rrbracket \| \mathrm{s} \llbracket S \rrbracket, X_{6}^{\prime}\right)\right\}
\end{aligned}
$$

Figure 7: An application of the inference algorithm to the hospital network, where $P, P_{1}, S$, $S_{1}, \mathrm{G}, \mathrm{G}_{1}$ and $\mathrm{G}_{2}$ are defined in the caption of Figure 4.

and $X \notin \operatorname{dom}(E)$. Indeed, we have $\mathbb{G} \neq$ End since players(End $\theta)=$ players(End) $=\emptyset$. First we show that $\mathbb{G}=X \in \operatorname{dom}(E)$ is impossible. In this case $X=\mathbb{G}_{1} \in E$ and we have $\mathbb{G} \theta=\mathbb{G}_{1} \theta$ and players $(\mathcal{S} ; E ; \mathbb{G})=\operatorname{players}\left(\mathcal{S} ; E ; \mathbb{G}_{1}\right)$, since $\theta$ is a solution of $E$. Hence, again $\mathbb{G}_{1} \neq$ End and if $\mathbb{G}_{1}=Y \in \operatorname{dom}(E)$, namely, $Y=\mathbb{G}_{2} \in E$, we have $\mathbb{G}_{1} \theta=\mathbb{G}_{2} \theta$ and players $\left(\mathcal{S} ; E ; \mathbb{G}_{1}\right)=\operatorname{players}\left(\mathcal{S} ; E ; \mathbb{G}_{2}\right)$ and, since $E$ is $\mathcal{S}$-closed and so guarded, we have that $\mathbb{G}_{2}$ is not a variable.

Case $d=0$ : If $\mathbb{G}^{\prime}=X \notin \operatorname{dom}(E)$, then $(\mathbb{N}, X) \in \mathcal{S}$ and players $\left(\mathcal{S} ; E ; \mathbb{G}^{\prime}\right)=\operatorname{players}(\mathbb{N})$. Since $\theta$ agrees with $\mathcal{S}$, we have players $\left(\mathbb{G}^{\prime} \theta\right)=\operatorname{players}(\theta(X))=$ players $(\mathbb{N})$, hence $\mathrm{p} \in \operatorname{players}\left(\mathcal{S} ; E ; \mathbb{G}^{\prime}\right)$. If $\mathbb{G}^{\prime}=\mathrm{rs} \dagger\left\{\lambda_{i} ; \mathbb{G}_{i}\right\}_{i \in I}$, then $\mathbb{G}^{\prime} \theta=\mathrm{rs} \dagger\left\{\lambda_{i} ; \mathbb{G}_{i} \theta\right\}_{i \in I}$ and 


$$
\begin{aligned}
& {\left[\mathrm{I}^{\prime}-\mathrm{END}\right] \overline{\mathcal{N} \vdash_{\mathrm{i}} \mathrm{p} \llbracket \mathbf{0} \rrbracket: \text { End }} \quad\left[\mathrm{I}^{\prime}-\mathrm{CYCLE}\right] \overline{\mathcal{N},(\mathbb{N}, \mathrm{G}) \vdash_{\mathrm{i}} \mathbb{N}: \mathrm{G}}} \\
& \mathcal{N},(\mathbb{N}, G) \vdash_{i} \mathrm{p} \llbracket P_{i} \rrbracket \| \mathbb{N}^{\prime}: G_{i} \\
& {\left[\mathrm{I}^{\prime} \text {-Out }\right] \frac{\text { players }\left(\mathrm{G}_{i}\right) \backslash\{\mathrm{p}\}=\operatorname{players}\left(\mathbb{N}^{\prime}\right) \quad \forall i \in I}{\mathcal{N} \vdash_{\mathbf{i}} \mathbb{N}: \mathrm{G}} \quad \begin{array}{c}
\mathrm{G}=\mathrm{qp} !\left\{\lambda_{i} ; \mathrm{G}_{i}\right\}_{i \in I} \\
\mathbb{N}=\mathrm{p} \llbracket \mathrm{q} !\left\{\lambda_{i} ; P_{i}\right\}_{i \in I} \rrbracket \| \mathbb{N}^{\prime}
\end{array}} \\
& \mathcal{N},(\mathbb{N}, \mathrm{G}) \vdash_{\mathrm{i}} \mathrm{p} \llbracket P_{i} \rrbracket \| \mathbb{N}^{\prime}: \mathrm{G}_{i} \\
& {\left[\mathrm{I}^{\prime}-\mathrm{IN}\right] \frac{\operatorname{players}\left(\mathrm{G}_{i}\right) \backslash\{\mathrm{p}\}=\operatorname{players}\left(\mathbb{N}^{\prime}\right) \quad \forall i \in I}{\mathcal{N} \vdash_{\mathrm{i}} \mathbb{N}: \mathrm{G}} \quad \begin{array}{c}
\mathrm{G}=\mathrm{qp} ?\left\{\lambda_{i} ; \mathrm{G}_{i}\right\}_{i \in I} \\
\mathbb{N}=\mathrm{p} \llbracket \mathrm{q} ?\left\{\lambda_{j} ; P_{j}\right\}_{j \in J} \rrbracket \| \mathbb{N}^{\prime} \\
I \subseteq J
\end{array}}
\end{aligned}
$$

Figure 8: Inductive typing rules for networks.

$\operatorname{play}\left(\mathbb{G}^{\prime}\right)=\operatorname{play}\left(\mathbb{G}^{\prime} \theta\right)=$ p. By definition we have players $\left(\mathcal{S} ; E ; \mathbb{G}^{\prime}\right)=\left\{\operatorname{play}\left(\mathbb{G}^{\prime}\right)\right\} \cup$ $\bigcup_{i \in I}$ players $\left(\mathcal{S}, E, \mathbb{G}_{i}\right)$, hence $\mathrm{p} \in \operatorname{players}\left(\mathcal{S} ; E ; \mathbb{G}^{\prime}\right)$.

Case $d>0$ : If $\mathbb{G}^{\prime}=X \notin \operatorname{dom}(E)$, the proof is as above. If $\mathbb{G}^{\prime}=\mathrm{rs} \dagger\left\{\lambda_{i} ; \mathbb{G}_{i}\right\}_{i \in I}$, then $\mathrm{p} \neq$ play $\left(\mathbb{G}^{\prime} \theta\right)$, hence $\mathrm{p} \neq \mathrm{r}$ if $\dagger=!$ and $\mathrm{p} \neq \mathrm{s}$ if $\dagger=$ ?. We have $\mathbb{G}^{\prime} \theta=\mathrm{rs} \dagger\left\{\lambda_{i} ; \mathbb{G}_{i} \theta\right\}_{i \in I}$ and there is $k \in I$ such that $\mathrm{p} \in \operatorname{players}\left(\mathbb{G}_{k} \theta\right)$ and the distance decreases. Then, by induction hypothesis, we get $\mathrm{p} \in \operatorname{players}\left(\mathcal{S} ; E ; \mathbb{G}_{k}\right) \subseteq \operatorname{players}\left(\mathcal{S} ; E ; \mathbb{G}^{\prime}\right)$, as needed.

To prove the other inclusion, players $(\mathcal{S} ; E ; \mathbb{G}) \subseteq$ players $(\mathbb{G} \theta)$, we just have to check that the sets players $(\mathbb{G} \theta)$ respect the equations defining players $(\mathcal{S} ; E ; \mathbb{G})$. All cases are trivial except for $\mathbb{G}=X$. If $X \in \operatorname{dom}(E)$, that is, $X=\mathbb{G}^{\prime} \in E$, then $\mathbb{G} \theta=\theta(X)=\mathbb{G}^{\prime} \theta$, hence players $(\mathbb{G} \theta)=$ players $\left(\mathbb{G}^{\prime} \theta\right)$, as needed. Otherwise, $X \in \operatorname{vars}(\mathcal{S})$, that is, $(\mathbb{N}, X) \in \mathcal{S}$, hence players $(\mathcal{S} ; E ; \mathbb{G})=\operatorname{players}(\mathbb{N})$. Since $\theta$ agrees with $\mathcal{S}$, we have players $(\mathbb{G} \theta)=\operatorname{players}(\theta(X))=$ players $(\mathbb{N})$, as needed.

To show soundness and completeness of our inference algorithm, it is handy to formulate an inductive version of our typing rules, see Figure 8, where $\mathcal{N}$ ranges over sets of pairs $(\mathbb{N}, G)$. We can give an inductive formulation since all infinite derivations using the typing rules of Figure 3 are regular, i.e. the number of different subtrees of a derivation for a judgement $G \vdash \mathbb{N}$ is finite. In fact, it is bounded by the product of the number of different subterms of $\mathrm{G}$ and the number of different subnetworks of $\mathbb{N}$, which are both finite as $\mathrm{G}$ and (processes in) $\mathbb{N}$ are regular. Applying the standard transformation according to [Dag21, Theorem 5.2] (see Definition 5.1 for the notational convention) from a coinductive to an inductive formulation we get the typing rules shown in Figure 8.

In the following two lemmas we relate inference and inductive derivability.

Lemma 3.15. If $\mathcal{S} \vdash(\mathbb{N}, X) \Rightarrow E$, then $\mathcal{S} \theta \vdash_{\mathrm{i}} \mathbb{N}: \theta(X)$ for all $\theta \in \operatorname{sol}_{\mathcal{S}}(E)$ such that $\operatorname{vars}(\mathcal{S}) \subseteq \operatorname{dom}(\theta)$.

Proof. By induction on the derivation of $\mathcal{S} \vdash(\mathbb{N}, X) \Rightarrow E$.

[I-END]: We have $E=\{X \equiv$ End $\}$, hence $\theta(X)=$ End and the thesis follows by rule [I'-END].

[I-CYCLE]: We have $E=\{X=Y\}$ and $\mathcal{S}=\mathcal{S}^{\prime},(\mathbb{N}, Y)$. Then, $\theta(X)=\theta(Y)$ and the thesis follows by Rule [I'-CYCLE].

[I-OUT]: We have $\mathbb{N} \equiv \mathrm{p} \llbracket \mathrm{q} !\left\{\lambda_{i} ; P_{i}\right\}_{i \in I} \rrbracket \| \mathbb{N}^{\prime}$ and $\mathcal{S},(\mathbb{N}, X) \vdash\left(\mathbb{N}_{i}, Y_{i}\right) \Rightarrow E_{i}$ with $Y_{i}$ fresh and $\mathbb{N}_{i} \equiv \mathrm{p} \llbracket P_{i} \rrbracket \| \mathbb{N}^{\prime}$ and players $\left(\mathcal{S},(\mathbb{N}, X) ; E_{i} ; Y_{i}\right) \backslash\{\mathrm{p}\}=$ players $\left(\mathbb{N}^{\prime}\right)$ for all $i \in I$ and $E=\left\{X \mp \mathrm{pq} !\left\{\lambda_{i} ; Y_{i}\right\}_{i \in I}\right\} \cup \bigcup_{i \in I} E_{i}$. Since $E_{i} \subseteq E$, we have $\theta \in \operatorname{sol}\left(E_{i}\right)$. Being $\theta \in \operatorname{sol}_{\mathcal{S}}(E)$, Lemma 3.14 implies players $(\mathcal{S} ; E ; X)=$ players $(\mathbb{N})$. So we get that $\theta$ agrees 
with $\mathcal{S},(\mathbb{N}, X)$. Then, by induction hypothesis, we have $\mathcal{S} \theta,(\mathbb{N}, \theta(X)) \vdash_{i} \mathbb{N}_{i}: \theta\left(Y_{i}\right)$ for all $i \in I$. The thesis follows by Rule [I'-OuT], since $\theta(X)=\mathrm{pq} !\left\{\lambda_{i} ; \theta\left(Y_{i}\right)\right\}_{i \in I}$ and players $\left(\mathcal{S},(\mathbb{N}, X) ; E_{i} ; Y_{i}\right) \backslash\{\mathbf{p}\}=\operatorname{players}\left(\mathbb{N}^{\prime}\right)$ implies players $\left(\theta\left(Y_{i}\right)\right) \backslash\{\mathbf{p}\}=\operatorname{players}\left(\mathbb{N}^{\prime}\right)$ for all $i \in I$ by Lemma 3.14.

[I-IN]: We have $\mathbb{N} \equiv \mathrm{p} \llbracket \mathrm{q} ?\left\{\lambda_{j} ; P_{j}\right\}_{j \in J} \rrbracket \| \mathbb{N}^{\prime}$ and $\mathcal{S},(\mathbb{N}, X) \vdash\left(\mathbb{N}_{i}, Y_{i}\right) \Rightarrow E_{i}$ with $Y_{i}$ fresh and $\mathbb{N}_{i} \equiv \mathrm{p} \llbracket P_{i} \rrbracket \| \mathbb{N}^{\prime}$ and players $\left(\mathcal{S},(\mathbb{N}, X) ; E_{i} ; Y_{i}\right) \backslash\{\mathrm{p}\}=$ players $\left(\mathbb{N}^{\prime}\right)$ for all $i \in I \subseteq J$, and $E=\left\{X=\mathrm{q}\right.$ p? $\left.\left\{\lambda_{i} ; Y_{i}\right\}_{i \in I}\right\} \cup \bigcup_{i \in I} E_{i}$. Since $E_{i} \subseteq E$, we have $\theta \in \operatorname{sol}\left(E_{i}\right)$. Being $\theta \in \operatorname{sol}_{\mathcal{S}}(E)$, Lemma 3.14 implies players $(\mathcal{S} ; E ; X)=$ players $(\mathbb{N})$. So we get that $\theta$ agrees with $\mathcal{S},(\mathbb{N}, X)$. Then, by induction hypothesis, we have $\mathcal{S} \theta,(\mathbb{N}, \theta(X)) \vdash_{i} \mathbb{N}_{i}: \theta\left(Y_{i}\right)$, for all $i \in I$. The thesis follows by Rule [I'-IN], since $\theta(X)=\mathrm{qp} ?\left\{\lambda_{i} ; \theta\left(Y_{i}\right)\right\}_{i \in I}$ and $\operatorname{players}\left(\mathcal{S},(\mathbb{N}, X) ; E_{i} ; Y_{i}\right) \backslash\{\mathrm{p}\}=\operatorname{players}\left(\mathbb{N}^{\prime}\right)$ implies players $\left(\theta\left(Y_{i}\right)\right) \backslash\{\mathrm{p}\}=\operatorname{players}\left(\mathbb{N}^{\prime}\right)$ for all $i \in I$ by Lemma 3.14.

Lemma 3.16. If $\mathcal{N} \vdash_{i} \mathbb{N}: G$ and players $\left(G^{\prime}\right)=\operatorname{players}\left(\mathbb{N}^{\prime}\right)$ for all $\left(\mathbb{N}^{\prime}, G^{\prime}\right) \in \mathcal{N}$, then, for all $\mathcal{S}, X$ and $\sigma$ such that $X \notin \operatorname{vars}(\mathcal{S}), \operatorname{dom}(\sigma)=\operatorname{vars}(\mathcal{S})$ and $\mathcal{S} \sigma=\mathcal{N}$, there are $E$ and $\theta$ such that $\mathcal{S} \vdash(\mathbb{N}, X) \Rightarrow E$ and $\theta \in \operatorname{sol}_{\mathcal{S}}(E)$ and $\operatorname{dom}(\theta)=\operatorname{vars}(E) \cup \operatorname{vars}(\mathcal{S})$ and $\sigma \preceq \theta$ and $\theta(X)=\mathrm{G}$.

Proof. By induction on the derivation of $\mathcal{N} \vdash_{i} \mathbb{N}: G$.

[I'-END]: The thesis is immediate by Rule [I-END] taking $\theta=\sigma+\{X \mapsto$ End $\}$.

[I'-CYCLE]: We have $\mathcal{N}=\mathcal{N}^{\prime},(\mathbb{N}, \mathrm{G})$, then $\mathcal{S}=\mathcal{S}^{\prime},(\mathbb{N}, Y)$ and $\sigma(Y)=\mathrm{G}$. By Rule [ICYCLE], we get $\mathcal{S} \vdash(\mathbb{N}, X) \Rightarrow\{X \mp Y\}$, hence $\theta=\sigma+\{X \mapsto \mathrm{G}\}$ is a solution of $\{X=Y\}$, which agrees with $\mathcal{S}$ being players $(\mathrm{G})=$ players $(\mathbb{N})$, as needed.

$\left[\mathbf{I}^{\prime}\right.$-OUT]: In this case we have $\mathbb{N} \equiv \mathrm{p} \llbracket \mathrm{q} !\left\{\lambda_{i} ; P_{i}\right\}_{i \in I} \rrbracket \| \mathbb{N}^{\prime}$ and $\mathbf{G}=\mathrm{pq} !\left\{\lambda_{i} ; \mathrm{G}_{i}\right\}_{i \in I}$ and $\mathcal{N},(\mathbb{N}, G) \vdash_{i} \mathbb{N}_{i}: G_{i}$ with $\mathbb{N}_{i} \equiv \mathrm{p} \llbracket P_{i} \rrbracket \| \mathbb{N}^{\prime}$ and players $\left(\mathrm{G}_{i}\right) \backslash\{\mathrm{p}\}=\operatorname{players}\left(\mathbb{N}^{\prime}\right)$, for all $i \in I$. This last condition implies players $(\mathrm{G})=$ players $(\mathbb{N})$. Set $\sigma^{\prime}=\sigma+\{X \mapsto \mathbf{G}\}$ and $\mathcal{S}^{\prime}=\mathcal{S},(\mathbb{N}, X)$, then, by induction hypothesis, we get that there are $E_{i}$ and $\theta_{i}$ such that $\mathcal{S}^{\prime} \vdash\left(\mathbb{N}_{i}, Y_{i}\right) \Rightarrow E_{i}$ and $\theta_{i} \in \operatorname{sol}_{\mathcal{S}^{\prime}}\left(E_{i}\right)$ and $\operatorname{dom}\left(\theta_{i}\right)=\operatorname{vars}\left(E_{i}\right) \cup \operatorname{vars}\left(\mathcal{S}^{\prime}\right)$ and $\sigma^{\prime} \preceq \theta_{i}$ and $\theta_{i}\left(Y_{i}\right)=\mathrm{G}_{i}$, for all $i \in I$. We can assume that $i \neq j$ implies $Y_{i} \neq Y_{j}$ and $\operatorname{dom}\left(E_{i}\right) \cap \operatorname{dom}\left(E_{j}\right)=\emptyset$ for all $i, j \in I$, because the algorithm always introduces fresh variables. This implies $\operatorname{dom}\left(\theta_{i}\right) \cap \operatorname{dom}\left(\theta_{j}\right)=\operatorname{vars}\left(\mathcal{S}^{\prime}\right)$ for all $i \neq j$, and so $\theta=\sum_{i \in I} \theta_{i}$ is well defined. Moreover, we have $\theta \in \operatorname{sol}_{\mathcal{S}^{\prime}}\left(E_{i}\right)$ and $\sigma \preceq \theta$ and $\theta(X)=\mathrm{G}$, as $\sigma \preceq \sigma^{\prime}$ and $\sigma^{\prime} \preceq \theta_{i} \preceq \theta$ for all $i \in I$. From players $\left(\mathrm{G}_{i}\right) \backslash\{\mathrm{p}\}=\operatorname{players}\left(\mathbb{N}^{\prime}\right)$ we get players $\left(\mathcal{S}^{\prime} ; E_{i} ; Y_{i}\right) \backslash\{\mathrm{p}\}=$ players $\left(\mathbb{N}^{\prime}\right)$ for all $i \in I$ by Lemma 3.14. By Rule [I-OUT] we get $\mathcal{S} \vdash(\mathbb{N}, X) \Rightarrow E$ with $E=\left\{X=\mathrm{pq} !\left\{\lambda_{i} ; Y_{i}\right\}_{i \in I}\right\} \cup \bigcup_{i \in I} E_{i}$ and $\theta \in \operatorname{sol}_{\mathcal{S}}(E)$, since $\theta(X)=\mathrm{pq} !\left\{\lambda_{i} ; \mathrm{G}_{i}\right\}_{i \in I}=\mathrm{pq} !\left\{\lambda_{i} ; \theta_{i}\left(Y_{i}\right)\right\}_{i \in I}=\left(\mathrm{pq} !\left\{\lambda_{i} ; Y_{i}\right\}_{i \in I}\right) \theta$ and $\sigma \preceq \theta$.

$\left[\mathbf{I}^{\prime}\right.$-IN]: In this case we have $\mathbb{N} \equiv \mathrm{p} \llbracket \mathrm{q} ?\left\{\lambda_{j} ; P_{j}\right\}_{j \in J} \rrbracket \| \mathbb{N}^{\prime}$ and $\mathrm{G}=\mathrm{qp} ?\left\{\lambda_{i} ; \mathrm{G}_{i}\right\}_{i \in I}$ with $I \subseteq J$ and $\mathcal{N},(\mathbb{N}, G) \vdash_{i} \mathbb{N}_{i}: G_{i}$ with $\mathbb{N}_{i} \equiv \mathrm{p} \llbracket P_{i} \rrbracket \| \mathbb{N}^{\prime}$ and players $\left(\mathrm{G}_{i}\right) \backslash\{\mathrm{p}\}=\operatorname{players}\left(\mathbb{N}^{\prime}\right)$, for all $i \in I$. This last condition implies players $(\mathrm{G})=$ players $(\mathbb{N})$. Set $\sigma^{\prime}=\sigma+\{X \mapsto \mathbf{G}\}$ and $\mathcal{S}^{\prime}=\mathcal{S},(\mathbb{N}, X)$, then, by induction hypothesis, we get that there are $E_{i}$ and $\theta_{i}$ such that $\mathcal{S}^{\prime} \vdash\left(\mathbb{N}_{i}, Y_{i}\right) \Rightarrow E_{i}$ and $\theta_{i} \in \operatorname{sol}_{\mathcal{S}^{\prime}}\left(E_{i}\right)$ and $\operatorname{dom}\left(\theta_{i}\right)=\operatorname{vars}\left(E_{i}\right) \cup \operatorname{vars}\left(\mathcal{S}^{\prime}\right)$ and $\sigma^{\prime} \preceq \theta_{i}$ and $\theta_{i}\left(Y_{i}\right)=\mathrm{G}_{i}$, for all $i \in I$. We can assume that $i \neq j$ implies $Y_{i} \neq Y_{j}$ and $\operatorname{dom}\left(E_{i}\right) \cap \operatorname{dom}\left(E_{j}\right)=\emptyset$ for all $i, j \in I$, because the algorithm always introduces fresh variables. This implies $\operatorname{dom}\left(\theta_{i}\right) \cap \operatorname{dom}\left(\theta_{j}\right)=\operatorname{vars}\left(\mathcal{S}^{\prime}\right)$ for all $i \neq j$, and so $\theta=\sum_{i \in I} \theta_{i}$ is well defined. Moreover, we have $\theta \in \operatorname{sol}_{\mathcal{S}^{\prime}}\left(E_{i}\right), \sigma \preceq \theta$ and $\theta(X)=\mathrm{G}$, as $\sigma \preceq \sigma^{\prime}$ and $\sigma^{\prime} \preceq \theta_{i} \preceq \theta$ for all $i \in I$. From players $\left(\mathrm{G}_{i}\right) \backslash\{\mathrm{p}\}=$ players $\left(\mathbb{N}^{\prime}\right)$ we get $\operatorname{players}\left(\mathcal{S}^{\prime} ; E_{i} ; Y_{i}\right) \backslash\{\mathrm{p}\}=\operatorname{players}\left(\mathbb{N}^{\prime}\right)$ for all $i \in I$ by Lemma 3.14. By Rule [I-IN], we 


$$
\begin{aligned}
& \text { get } \mathcal{S} \vdash(\mathbb{N}, X) \Rightarrow E \text { with } E=\left\{X=\mathrm{qp} ?\left\{\lambda_{i} ; Y_{i}\right\}_{i \in I}\right\} \cup \bigcup_{i \in I} E_{i} \text { and } \theta \in \operatorname{sol}_{\mathcal{S}}(E), \text { since } \\
& \theta(X)=\mathrm{qp} ?\left\{\lambda_{i} ; \mathrm{G}_{i}\right\}_{i \in I}=\mathrm{qp} ?\left\{\lambda_{i} ; \theta_{i}\left(Y_{i}\right)\right\}_{i \in I}=\left(\mathrm{qp} ?\left\{\lambda_{i} ; Y_{i}\right\}_{i \in I}\right) \theta \text { and } \sigma \preceq \theta .
\end{aligned}
$$

Theorem 3.17 (Soundness and completeness of inference).

(1) If $\vdash(\mathbb{N}, X) \Rightarrow E$, then $\theta(X) \vdash \mathbb{N}$ for all $\theta \in \operatorname{sol}(E)$.

(2) If $\mathrm{G} \vdash \mathbb{N}$, then there are $E$ and $\theta$ such that $\vdash(\mathbb{N}, X) \Rightarrow E$ and $\theta \in \operatorname{sol}(E)$ and $\theta(X)=\mathrm{G}$.

Proof. (1). By Lemma 3.15 $\vdash(\mathbb{N}, X) \Rightarrow E$ implies $\vdash_{i} \mathbb{N}: \theta(X)$ for all $\theta \in \operatorname{sol}(E)$. This is enough, since $\vdash_{i} \mathbb{N}: \theta(X)$ gives $\theta(X) \vdash \mathbb{N}$.

$(2)$. From $\mathrm{G} \vdash \mathbb{N}$ we get $\vdash_{\mathbf{i}} \mathbb{N}: G$. By Lemma 3.16 this implies that there are $E$ and $\theta$ such that $\vdash(\mathbb{N}, X) \Rightarrow E$ and $\theta \in \operatorname{sol}(E)$ and $\theta(X)=\mathrm{G}$.

Remark 3.18 (Termination). As happens for (co)SLD-resolution in logic programming, the termination of our inference algorithm depends on the choice of a resolution strategy. Indeed, we have many sources of non-determinism: we have to select a goal to be resolved and, for each of such goals, we can either pick a participant of the network and expand it using rules [I-IN] or [I-OUT], or try to close a cycle using the rule [I-CYCLE]. A standard way to obtain a sound and complete resolution strategy is to build a tree where all such choices are performed in parallel and then visit the tree using a breadth-first strategy. The tree is potentially infinite in depth, but it is finitely branching, since at each point we have only finitely many different choices, hence this strategy necessarily enumerates all solutions. Moreover, by Theorem 3.8, we know that every network has a type, therefore this strategy necessarily finds a solution, that is, it terminates. Notice that the same network can be typed by a weakly balanced global type and a non weakly balanced global type (weak balancing is defined in Figure 9). For instance the hospital network can be typed by the weakly balanced global type of Figure 4 and the non weakly balanced global types of Example 3.6.

\section{TAMing Types}

In this section we tame global types so that the resulting typing enforces the required properties, i.e. Subject Reduction and Progress. We start by characterising a class of global types for which, when looking at global types as trees, the first occurrences of players are at a bounded depth in all paths starting from arbitrary nodes. This property, that we call boundedness, is required by the transition Rules [INSIDE-OUT] and [INSIDE-In]. It is also needed for Subject Reduction and Progress. We then pass to formalise a condition on type

configurations inspired again by the "inside" rules of the LTS in Figure 5. We require that for each choice of inputs there should be either an output or a message in the queue matching one of the choice labels. We call this property weak balancing, since it only requires matching for inputs. Instead, we call balanced a type configuration in which, in addition to the weak balancing, each output emitted by the global type and each message in the queue must have an input reading it in the type. For a session $\mathbb{N} \| \mathcal{M}$ whose network is typed by a global type $G$, boundedness of $G$ and weak balancing of $G \| \mathcal{M}$ suffice to prove Subject Reduction and the properties of deadlock freedom and input lock-freedom. To prove orphan-message freedom, as expected, we also need balancing of $\mathrm{G} \| \mathcal{M}$. 
4.1. Boundedness. To formalise boundedness we use $\xi$ to denote a path in global type trees, i.e., a possibly infinite sequence of communications $\mathrm{pq} ! \lambda$ or $\mathrm{pq}$ ? $\lambda$. With $\xi_{n}$ we represent the $n$-th communication in the path $\xi$, where $0 \leq n<x$ and $x \in \mathbf{N} \cup\{\omega\}$ is the length of $\xi$. By $\epsilon$ we denote the empty sequence and by $\cdot$ the concatenation of a finite sequence with a possibly infinite sequence. The function Paths gives the set of paths of global types, which are the greatest sets such that:

$$
\begin{aligned}
\text { Paths }(\text { End }) & =\{\epsilon\} \\
\operatorname{Paths}\left(\mathrm{pq} \dagger\left\{\lambda_{i} ; \mathrm{G}_{i}\right\}_{i \in I}\right) & =\bigcup_{i \in I}\left\{\mathrm{pq} \dagger \lambda_{i} \cdot \xi \mid \xi \in \operatorname{Paths}\left(\mathrm{G}_{i}\right)\right\}
\end{aligned}
$$

It is handy to define the depth of a player $\mathrm{p}$ in a global type $\mathrm{G}, \operatorname{depth}(\mathrm{G}, \mathrm{p})$.

Definition 4.1 (Depth of a player). Let $\mathrm{G}$ be a global type. For $\xi \in \operatorname{Paths}(\mathrm{G}) \operatorname{set} \operatorname{depth}(\xi, \mathrm{p})=$ $\inf \left\{n \mid \operatorname{play}\left(\xi_{n}\right)=\mathrm{p}\right\}$, and $\operatorname{define} \operatorname{depth}(\mathrm{G}, \mathrm{p})$, the depth of $\mathrm{p}$ in $\mathrm{G}$, as follows:

$$
\operatorname{depth}(\mathrm{G}, \mathrm{p})= \begin{cases}1+\sup \{\operatorname{depth}(\xi, \mathrm{p}) \mid \xi \in \operatorname{Paths}(\mathrm{G})\} & \text { if } \mathrm{p} \in \operatorname{players}(\mathrm{G}) \\ 0 & \text { otherwise }\end{cases}
$$

Note that $\operatorname{depth}(\mathrm{G}, \mathrm{p})=0$ iff $\mathrm{p} \notin$ players $(\mathrm{G})$. Moreover, if $\mathrm{p} \neq \operatorname{play}\left(\xi_{n}\right)$ for some path $\xi$ and all $n \in \mathbf{N}$, then $\operatorname{depth}(\xi, \mathrm{p})=\inf \emptyset=\infty$. Hence, if $\mathrm{p}$ is a player of a global type $\mathbf{G}$, but it does not occur as a player in some path of $G$, then $\operatorname{depth}(G, p)=\infty$.

We can now define the condition we were looking for.

Definition 4.2 (Boundedness). A global type $G$ is bounded if $\operatorname{depth}\left(G^{\prime}, p\right)$ is finite for all participants $p \in$ players $\left(\mathrm{G}^{\prime}\right)$ and all types $\mathrm{G}^{\prime}$ which occur in $\mathrm{G}$.

Example 4.3. The following example shows the necessity of considering all types occurring in a global type for defining boundedness. Consider $G=r q ! \lambda ; r q ? \lambda ; G^{\prime}$, where

$$
\mathrm{G}^{\prime}=\mathrm{pq} !\left\{\lambda_{1} ; \mathrm{pq} ? \lambda_{1} ; \mathrm{qr} ! \lambda_{3} ; \mathrm{qr} ? \lambda_{3}, \lambda_{2} ; \mathrm{pq} ? \lambda_{2} ; \mathrm{G}^{\prime}\right\}
$$

Then we have:

$$
\operatorname{depth}(\mathrm{G}, \mathrm{r})=1 \quad \operatorname{depth}(\mathrm{G}, \mathrm{p})=3 \quad \operatorname{depth}(\mathrm{G}, \mathrm{q})=2
$$

whereas

$$
\operatorname{depth}\left(G^{\prime}, r\right)=\infty \quad \operatorname{depth}\left(G^{\prime}, p\right)=1 \quad \operatorname{depth}\left(G^{\prime}, q\right)=2
$$

Since global types are regular the boundedness condition is decidable.

It is easy to check that the LTS of type configurations preserves the boundedness of global types.

Proposition 4.4. If $\mathrm{G}$ is bounded and $\mathrm{G}\left\|\mathcal{M} \stackrel{\beta}{\rightarrow} \mathrm{G}^{\prime}\right\| \mathcal{M}^{\prime}$, then $\mathrm{G}^{\prime}$ is bounded.

Proof. By induction on the transition rules of Figure 5. 
4.2. Balancing. The weak balancing predicate of Figure 9 ensures that at least one of the labels of every input choice of the global type is matched either by a message in the queue or by a preceding output in the global type. Rule [WB-OUT] removes an output choice from the type and puts the corresponding message in the queue. This allows a subsequent input choice to be matched by one of its output messages. Rule [WB-IN] says that every initial input choice should find one of its corresponding messages in the queue. Notice the similarity between these two rule and Rules [Inside-OUT], [INSIDE-IN] of Figure 5.

Example 4.5. Figure 10 displays the initial part of the weakly balancing derivation for the type configuration $G \| \emptyset$, where $G, G_{1}$ and $G_{2}$ are defined in the caption of Figure 4.

$$
\begin{aligned}
& \text { [WB-END] } \underset{\vdash_{\mathrm{wb}} \text { End } \| \emptyset}{\bar{C} \emptyset \mathrm{WB}-\mathrm{OUT}]} \frac{\vdash_{\mathrm{wb}} \mathrm{G}_{i} \| \mathcal{M} \cdot\left\langle\mathrm{p}, \lambda_{i}, \mathrm{q}\right\rangle \quad \forall i \in I}{\vdash_{\mathrm{wb}} \mathrm{pq} !\left\{\lambda_{i} ; \mathrm{G}_{i}\right\}_{i \in I} \| \mathcal{M}} \\
& \text { [WB-IN] } \frac{\vdash_{\mathrm{wb}} \mathrm{G}_{h} \| \mathcal{M}}{\vdash_{\mathrm{wb}} \mathrm{pq} ?\left\{\lambda_{i} ; \mathrm{G}_{i}\right\}_{i \in I} \|\left\langle\mathrm{p}, \lambda_{h}, \mathrm{q}\right\rangle \cdot \mathcal{M}} h \in I
\end{aligned}
$$

Figure 9: Weak balancing of type configurations.

The following proposition shows that reduction of global types preserves weak balancing.

Proposition 4.6. If $\vdash_{\mathrm{wb}} \mathrm{G} \| \mathcal{M}$ and $\mathrm{G}\left\|\mathcal{M} \stackrel{\beta}{\rightarrow} \mathrm{G}^{\prime}\right\| \mathcal{M}^{\prime}$, then $\vdash_{\mathrm{wb}} \mathrm{G}^{\prime} \| \mathcal{M}^{\prime}$.

Proof. By induction on the transition rules of Figure 5.

With boundedness of global types and weak balancing of type configurations we can prove the expected Subject Reduction Theorem.

$$
\begin{aligned}
& \text { D } \\
& \frac{\overline{\frac{\vdash_{\mathrm{wb}} \mathrm{G}_{1} \|\langle\mathrm{p}, p r, \mathrm{~s}\rangle \cdot\langle\mathrm{p}, n d, \mathrm{~s}\rangle}{}}}{\vdash_{\mathrm{wb}} \mathrm{G} \|\langle\mathrm{p}, p r, \mathrm{~s}\rangle} \\
& \frac{\vdash_{\mathrm{wb}} \mathrm{G} \| \emptyset}{\overline{\vdash_{\mathrm{wb}} \mathrm{sp} ? o k ; \mathrm{G} \|\langle\mathrm{s}, o k, \mathrm{p}\rangle}} \quad \frac{\overline{\vdash_{\mathrm{wb}} \mathrm{ps} ! p r ; \mathrm{G} \| \emptyset}}{\overline{\vdash_{\mathrm{wb}} \mathrm{sp} ? k o ; \mathrm{ps} ! p r ; \mathrm{G} \|\langle\mathrm{s}, k o, \mathrm{p}\rangle}} \\
& \frac{\vdash_{\text {wb } \mathrm{sp} ? o k ; \mathrm{G} \|\langle\mathrm{s}, o k, \mathrm{p}\rangle} \vdash_{\mathrm{wb}} \mathrm{sp} ? k o ; \mathrm{ps} ! p r ; \mathrm{G} \|\langle\mathrm{s}, k o, \mathrm{p}\rangle}{\vdash_{\mathrm{wb}} \mathrm{G}_{2} \| \emptyset} \\
& \frac{\overline{\frac{\vdash_{\mathrm{wb}} \mathrm{G}_{1} \|\langle\mathrm{p}, n d, \mathrm{~s}\rangle}{\vdash_{\mathrm{wb}} \mathrm{G} \| \emptyset}}}{\overline{\vdash_{\mathrm{w}} \emptyset}}
\end{aligned}
$$

where

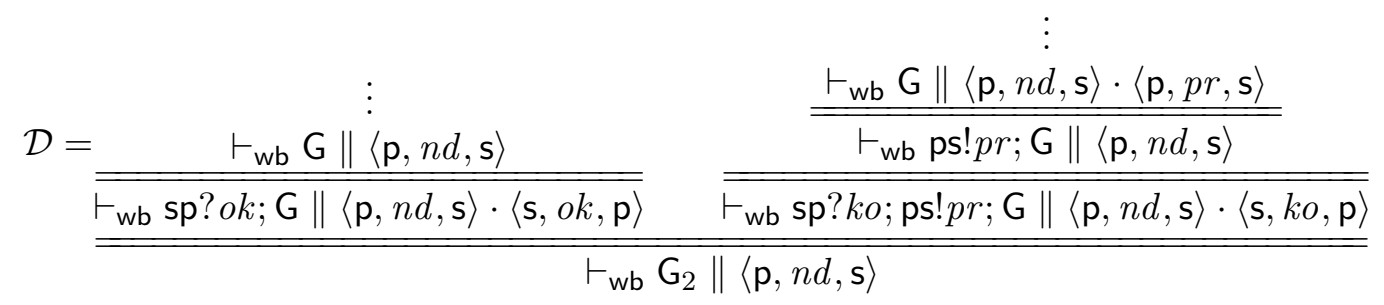

Figure 10: Weak balancing of the hospital global type with the empty queue. 
Theorem 4.7 (Subject Reduction). If $\mathrm{G} \vdash \mathbb{N}$ and $\mathbb{N}\left\|\mathcal{M} \stackrel{\beta}{\rightarrow} \mathbb{N}^{\prime}\right\| \mathcal{M}^{\prime}$ and $\mathrm{G}$ is bounded and $\vdash_{\text {wb }} \mathrm{G} \| \mathcal{M}$, then $\mathrm{G}\left\|\mathcal{M} \stackrel{\beta}{\rightarrow} \mathrm{G}^{\prime}\right\| \mathcal{M}^{\prime}$ and $\mathrm{G}^{\prime} \vdash \mathbb{N}^{\prime}$.

Proof. The proof is by induction on $d=\operatorname{depth}(\mathrm{G}, \mathrm{p})$ where $\mathrm{p}=\operatorname{play}(\beta)$. Notice that $\mathbb{N}\left\|\mathcal{M} \stackrel{\beta}{\rightarrow} \mathbb{N}^{\prime}\right\| \mathcal{M}^{\prime}$ implies $\mathrm{p} \in \operatorname{players}(\mathbb{N})$, which with $\mathrm{G} \vdash \mathbb{N}$ gives $\mathrm{p} \in \operatorname{players}(\mathrm{G})$ by Lemma 3.2. Then $d>0$. Moreover $d$ is finite since $\mathrm{G}$ is bounded.

Case $d=1$. If $\beta=\mathrm{qp}$ ? $\lambda$, then $\mathbb{N} \equiv \mathrm{p} \llbracket \mathrm{q} ?\left\{\lambda_{i} ; P_{i}\right\}_{i \in I} \rrbracket \| \mathbb{N}_{0}$ and $\mathbb{N}^{\prime}=\mathrm{p} \llbracket P_{h} \rrbracket \| \mathbb{N}_{0}$ and $\mathcal{M} \equiv\langle\mathbf{q}, \lambda, \mathbf{p}\rangle \cdot \mathcal{M}^{\prime}$ and $\lambda=\lambda_{h}$ for some $h \in I$. From $d=1$ we get $\mathbf{G}=\mathbf{q} \mathbf{p} ?\left\{\lambda_{j} ; \mathrm{G}_{j}\right\}_{j \in J}$ and $\mathrm{G}_{j} \vdash \mathrm{p} \llbracket P_{j} \rrbracket \| \mathbb{N}_{0}$ for all $j \in J$ with $J \subseteq I$. From $\mathcal{M} \equiv\langle\mathrm{q}, \lambda, \mathrm{p}\rangle \cdot \mathcal{M}^{\prime}$ and $\vdash_{\text {wb }} \mathrm{G} \| \mathcal{M}$ we get $h \in J$. We conclude $\mathrm{G}\left\|\mathcal{M} \stackrel{\beta}{\rightarrow} \mathrm{G}_{h}\right\| \mathcal{M}^{\prime}$. If $\beta=\mathrm{pq} ! \lambda$ the proof is similar and simpler.

Case $d>1$. Then either $\mathrm{G}=\mathrm{rq} !\left\{\lambda_{i} ; \mathrm{G}_{i}\right\}_{i \in I}$ or $\mathrm{G}=\mathrm{q} r ?\left\{\lambda_{i} ; \mathrm{G}_{i}\right\}_{i \in I}$ with $\mathrm{r} \neq \mathrm{p}=\operatorname{play}(\beta)$. Let $\mathbf{G}=\mathrm{rq} !\left\{\lambda_{i} ; \mathrm{G}_{i}\right\}_{i \in I}$, the proof for $\mathbf{G}=\mathrm{q} r ?\left\{\lambda_{i} ; \mathrm{G}_{i}\right\}_{i \in I}$ is similar. Since $\mathbf{G} \vdash \mathbb{N}$ must be derived using Rule [OUT] we get $\mathbb{N} \equiv \mathrm{r} \llbracket \mathrm{q} !\left\{\lambda_{i} ; R_{i}\right\}_{i \in I} \rrbracket \| \mathbb{N}_{0}$ and $\mathrm{G}_{i} \vdash \mathrm{r} \llbracket R_{i} \rrbracket \| \mathbb{N}_{0}$ for all $i \in I$. The condition $r \neq \operatorname{play}(\beta)$ ensures that the reduction $\mathbb{N}\left\|\mathcal{M} \stackrel{\beta}{\rightarrow} \mathbb{N}^{\prime}\right\| \mathcal{M}^{\prime}$ does not modify the process of participant $\mathrm{r}$, then we get $\mathbb{N}^{\prime} \equiv \mathrm{r} \llbracket \mathrm{q} !\left\{\lambda_{i} ; R_{i}\right\}_{i \in I} \rrbracket \| \mathbb{N}_{0}^{\prime}$. Moreover, the reduction can be done also if messages are added at the end of the queue. Therefore

$$
\mathrm{r} \llbracket R_{i} \rrbracket\left\|\mathbb{N}_{0}\right\| \mathcal{M} \cdot\left\langle\mathrm{r}, \lambda_{i}, \mathrm{q}\right\rangle \stackrel{\beta}{\rightarrow} \mathrm{r} \llbracket R_{i} \rrbracket\left\|\mathbb{N}_{0}^{\prime}\right\| \mathcal{M}^{\prime} \cdot\left\langle\mathrm{r}, \lambda_{i}, \mathrm{q}\right\rangle
$$

for all $i \in I$. From $\mathrm{G}$ bounded we have $\mathrm{G}_{i}$ bounded and from $\vdash_{\mathrm{wb}} \mathrm{G} \| \mathcal{M}$ we have $\vdash_{\mathrm{wb}} \mathrm{G}_{i} \| \mathcal{M} \cdot\left\langle\mathrm{r}, \lambda_{i}, \mathrm{q}\right\rangle$ by Rule [WB-OuT] for all $i \in I$. Since $\operatorname{depth}\left(\mathrm{G}_{i}, \mathrm{p}\right)<d$ for all $i \in I$, by induction we get $\mathrm{G}_{i}\left\|\mathcal{M} \cdot\left\langle\mathrm{r}, \lambda_{i}, \mathrm{q}\right\rangle \stackrel{\beta}{\rightarrow} \mathrm{G}_{i}^{\prime}\right\| \mathcal{M}^{\prime} \cdot\left\langle\mathrm{r}, \lambda_{i}, \mathrm{q}\right\rangle$ and $\mathrm{G}_{i}^{\prime} \vdash \mathrm{r} \llbracket R_{i} \rrbracket \| \mathbb{N}_{0}^{\prime}$ for some $\mathrm{G}_{i}^{\prime}$ and for all $i \in I$. We get $\mathrm{G}\left\|\mathcal{M} \stackrel{\beta}{\rightarrow} \mathrm{rq} !\left\{\lambda_{i} ; \mathrm{G}_{i}^{\prime}\right\}_{i \in I}\right\| \mathcal{M}^{\prime}$ using Rule [Inside-Out]. By Lemma $3.2 \mathrm{G}_{i}^{\prime} \vdash \mathrm{r} \llbracket R_{i} \rrbracket \| \mathbb{N}_{0}^{\prime}$ implies players $\left(\mathrm{G}_{i}^{\prime}\right) \backslash\{\mathrm{r}\}=\operatorname{players}\left(\mathbb{N}_{0}^{\prime}\right)$ for all $i \in I$. Then we can derive $\mathrm{r} \mathrm{q} !\left\{\lambda_{i} ; \mathrm{G}_{i}^{\prime}\right\}_{i \in I} \vdash \mathbb{N}^{\prime}$ using Rule [OUT].

Deadlock-freedom easily follows from Subject Reduction and Session Fidelity.

Theorem 4.8 (Deadlock-freedom). If $\mathrm{G} \vdash \mathbb{N}$ and $\mathrm{G}$ is bounded and $\vdash_{\mathrm{wb}} \mathrm{G} \| \mathcal{M}$, then $\mathbb{N} \| \mathcal{M}$ is deadlock free.

Proof. By Subject Reduction (Theorem 4.7) and Session Fidelity (Theorem 3.9) it is enough to show that $G \| \mathcal{M}$ is deadlock-free. One of the two Rules [Top-OUT] and [TOP-IN] is applicable whenever $\vdash_{w b} \mathrm{G} \| \mathcal{M}$ holds and $\mathrm{G}$ is not End.

Our next goal is to show that no player offering a choice of inputs can wait forever. To this aim we prove Subject Reduction for the lockstep transition relation. We dub this result Strong Subject Reduction.

Theorem 4.9 (Strong Subject Reduction). If $\mathrm{G} \vdash \mathbb{N}$ and $\mathrm{G}$ is bounded and $\vdash_{\mathrm{wb}} \mathrm{G} \| \mathcal{M}$ and $\mathbb{N}\left\|\mathcal{M} \triangleq \mathbb{N}^{\prime}\right\| \mathcal{M}^{\prime}$, then $\mathrm{G}\left\|\mathcal{M} \triangleq \mathrm{G}^{\prime}\right\| \mathcal{M}^{\prime}$ and $\mathrm{G}^{\prime} \vdash \mathbb{N}^{\prime}$.

Proof. Let $\Delta=\left\{\beta_{1}, \ldots, \beta_{n}\right\}$. By definition of $\triangleq$, the set $\Delta$ is coherent for $\mathbb{N} \| \mathcal{M}$, that is, we know that, for all $i \in 1 . . n, \mathbb{N} \| \mathcal{M} \stackrel{\beta_{i}}{\longrightarrow}$, hence, by Theorem 4.7, we get, for all $i \in 1 . . n$, $\mathrm{G} \| \mathcal{M} \stackrel{\beta_{i}}{\longrightarrow}$, namely $\Delta$ is coherent for $\mathrm{G} \| \mathcal{M}$. Consider a coherent set $\Delta^{\prime}$ for $\mathrm{G} \| \mathcal{M}$ such that $\Delta \subseteq \Delta^{\prime}$, then for all $\beta \in \Delta^{\prime}$, by Session Fidelity (Theorem 3.9), we get $\mathbb{N} \| \mathcal{M} \stackrel{\beta}{\rightarrow}$. Hence $\Delta^{\prime}$ 
is coherent for $\mathbb{N} \| \mathcal{M}$ and, since $\Delta$ is a maximal coherent set for $\mathbb{N} \| \mathcal{M}$ again by definition of $\triangleq$, we get $\Delta=\Delta^{\prime}$, that is, $\Delta$ is a maximal coherent set for $\mathrm{G} \| \mathcal{M}$.

Finally, since $\mathbb{N}\left\|\mathcal{M} \stackrel{\Delta}{\Rightarrow} \mathbb{N}^{\prime}\right\| \mathcal{M}^{\prime}$, we know that, for all $i \in 1 . . n, \mathbb{N}_{i-1}\left\|\mathcal{M}_{i-1} \stackrel{\beta_{i}}{\longrightarrow} \mathbb{N}_{i}\right\|$ $\mathcal{M}_{i}$, with $\mathbb{N}_{0}=\mathbb{N}, \mathcal{M}_{0}=\mathcal{M}, \mathbb{N}_{n}=\mathbb{N}^{\prime}$ and $\mathcal{M}_{n}=\mathcal{M}^{\prime}$. By Subject Reduction (Theorem 4.7) and Propositions 4.4, 4.6, we get, for all $i \in 1 . . n, \mathrm{G}_{i-1}\left\|\mathcal{M}_{i-1} \stackrel{\beta_{i}}{\longrightarrow} \mathrm{G}_{i}\right\| \mathcal{M}_{i}$ with $\mathrm{G}_{0}=\mathrm{G}$, $\vdash_{\mathrm{wb}} \mathrm{G}_{i} \| \mathcal{M}_{i}$ and $\mathrm{G}_{i} \vdash \mathbb{N}_{i}$. Hence, setting $\mathrm{G}^{\prime}=\mathrm{G}_{n}$, we get $\mathrm{G}\left\|\mathcal{M} \triangleq \mathrm{G}^{\prime}\right\| \mathcal{M}^{\prime}$ and $\mathrm{G}^{\prime} \vdash \mathbb{N}^{\prime}$.

We need a lemma showing that a communication labelling a transition of a type configuration with global type $\mathrm{G}$ must occur in all the paths of the tree representing $\mathrm{G}$.

Lemma 4.10. If $\mathrm{G}\left\|\mathcal{M} \stackrel{\beta}{\rightarrow} \mathrm{G}^{\prime}\right\| \mathcal{M}^{\prime}$, then for all paths $\xi \in \operatorname{Paths}\left(\mathrm{G}^{\prime}\right), \xi=\xi_{1} \cdot \xi_{2}$ and $\xi_{1} \cdot \beta \cdot \xi_{2} \in \operatorname{Paths}(\mathrm{G})$, for some $\xi_{1}, \xi_{2}$.

Proof. By induction on the rules defining the transition relation, see Figure 5.

A last lemma before establishing the property of "input-enabling" relates the depth of players in global types with the reductions of the corresponding type configurations.

Lemma 4.11. Let $\mathrm{G}$ be bounded and $\vdash_{\mathrm{wb}} \mathrm{G} \| \mathcal{M}$ and $\mathrm{p} \in$ players $(\mathrm{G})$, then the following hold:

(1) If $\operatorname{depth}(\mathrm{G}, \mathrm{p})=1$, then $\mathrm{G}\left\|\mathcal{M} \stackrel{\beta}{\rightarrow} \mathrm{G}^{\prime}\right\| \mathcal{M}^{\prime}$, with $\operatorname{play}(\beta)=\mathrm{p}$.

(2) If $\mathrm{G}\left\|\mathcal{M} \stackrel{\beta}{\rightarrow} \mathrm{G}^{\prime}\right\| \mathcal{M}^{\prime}$, with $\mathrm{p} \neq \operatorname{play}(\beta)$, then $\operatorname{depth}\left(\mathrm{G}^{\prime}, \mathrm{p}\right) \leq \operatorname{depth}(\mathrm{G}, \mathrm{p})$.

(3) If $\mathrm{G}\left\|\mathcal{M} \triangleq \mathrm{G}^{\prime}\right\| \mathcal{M}^{\prime}$, with $\mathrm{p} \neq \operatorname{play}(\beta)$ for all $\beta \in \Delta$, then $\operatorname{depth}\left(\mathrm{G}^{\prime}, \mathrm{p}\right)<\operatorname{depth}(\mathrm{G}, \mathrm{p})$.

Proof. (1). If $\operatorname{depth}(\mathrm{G}, \mathrm{p})=1$ there are two possibilities: either $\mathrm{G}=\mathrm{pq} !\left\{\lambda_{i} ; \mathrm{G}_{i}\right\}_{i \in I}$ or $\mathrm{G}=\mathrm{qp} ?\left\{\lambda_{i} ; \mathrm{G}_{i}^{\prime}\right\}_{i \in I}$. In the first case, $\mathrm{G}\left\|\mathcal{M} \stackrel{\mathrm{pq} ! \lambda_{h}}{\longrightarrow} \mathrm{G}_{h}\right\| \mathcal{M}^{\prime}$, for some $h \in I$, by Rule [Top-Out]. In the second case, from $\mathrm{G} \| \mathcal{M}$ weakly balanced we get $\mathcal{M} \equiv\left\langle\mathbf{q}, \lambda_{h}, \mathrm{p}\right\rangle \cdot \mathcal{M}^{\prime}$ and $\mathrm{G}\left\|\mathcal{M} \stackrel{\mathrm{qp} ? \lambda_{h}}{\longrightarrow} \mathrm{G}_{h}\right\| \mathcal{M}^{\prime}$, for some $h \in I$, by Rule [TOP-IN].

(2). Let $\xi \in \operatorname{Paths}\left(\mathrm{G}^{\prime}\right)$, then by Lemma 4.10 , we have $\xi=\xi_{1} \cdot \xi_{2}$ and $\xi_{1} \cdot \beta \cdot \xi_{2} \in \operatorname{Paths}(\mathrm{G})$, for some $\xi_{1}, \xi_{2}$. Since $\mathrm{G}$ is bounded, $\operatorname{depth}(\mathrm{G}, \mathrm{p})$ is finite, hence there is a first occurrence of $\beta^{\prime}$ with play $\left(\beta^{\prime}\right)=\mathrm{p}$ either in $\xi_{1}$ or in $\xi_{2}$, because play $(\beta) \neq \mathrm{p}$. In the former case we have $\operatorname{depth}(\xi, \mathrm{p})=\operatorname{depth}\left(\xi_{1} \cdot \beta \cdot \xi_{2}, \mathrm{p}\right) \leq \operatorname{depth}(\mathrm{G}, \mathrm{p})$, and, in the latter case, we have $\operatorname{depth}(\xi, \mathrm{p})<\operatorname{depth}\left(\xi_{1} \cdot \beta \cdot \xi_{2}, \mathrm{p}\right) \leq \operatorname{depth}(\mathrm{G}, \mathrm{p})$. This implies

$$
\operatorname{depth}\left(\mathrm{G}^{\prime}, \mathrm{p}\right)=\sup \left\{\operatorname{depth}\left(\xi^{\prime}, \mathrm{p}\right) \mid \xi^{\prime} \in \operatorname{Paths}\left(\mathrm{G}^{\prime}\right)\right\} \leq \operatorname{depth}(\mathrm{G}, \mathrm{p})
$$

as needed.

(3). We have two cases on $\mathrm{G}$.

- If $\mathrm{G}=\mathrm{q} r !\left\{\lambda_{i} ; \mathrm{G}_{i}\right\}_{i \in I}$, then, by Rule [TOP-OUT], $\mathrm{G}\|\mathcal{M} \stackrel{\beta}{\rightarrow} \widehat{\mathrm{G}}\| \widehat{\mathcal{M}}$ where $\beta=\mathrm{q} r ! \lambda_{h} \in \Delta$ and $\widehat{G}=G_{h}$ for some $h \in I$. From $\Delta$ maximal we get $\beta \in \Delta$. Then, for all paths $\xi \in \operatorname{Paths}(\widehat{\mathrm{G}})$, we have $\beta \cdot \xi \in \operatorname{Paths}(\mathrm{G})$. Since $\operatorname{depth}(\mathrm{G}, \mathrm{p})$ is finite, because $\mathrm{G}$ is bounded, and $\mathrm{p} \neq \operatorname{play}(\beta)$, being $\mathrm{p} \neq \operatorname{play}\left(\beta^{\prime}\right)$ for all $\beta^{\prime} \in \Delta$, there is in $\xi$ a first occurrence of $\beta_{0}$ with play $\left(\beta_{0}\right)=\mathrm{p}$. Thus we $\operatorname{get} \operatorname{depth}(\xi, \mathrm{p})<\operatorname{depth}(\beta \cdot \xi, \mathrm{p})$. This implies $\operatorname{depth}(\widehat{\mathrm{G}}, \mathrm{p})<$ $\operatorname{depth}(\mathrm{G}, \mathrm{p})$.

- If $\mathrm{G}=\mathrm{q}$ r? $\left\{\lambda_{i} ; \mathrm{G}_{i}\right\}_{i \in I}$, then $\vdash_{\mathrm{wb}} \mathrm{G} \| \mathcal{M}$ implies $\mathcal{M} \equiv\left\langle\mathrm{q}, \lambda_{h}, \mathrm{r}\right\rangle \cdot \widehat{\mathcal{M}}$ for some $h \in I$. By Rule [Top-In], G $\|\mathcal{M} \stackrel{\beta}{\rightarrow} \widehat{\mathrm{G}}\| \widehat{\mathcal{M}}$ where $\beta=\mathrm{qr}$ ? $\lambda_{h}$. From $\Delta$ maximal we get $\beta \in \Delta$. Then, 
for all paths $\xi \in \operatorname{Paths}(\widehat{\mathrm{G}})$, we have $\beta \cdot \xi \in \operatorname{Paths}(\mathrm{G})$. Since $\operatorname{depth}(\mathrm{G}, \mathrm{p})$ is finite, because $\mathrm{G}$ is bounded, and $\mathrm{p} \neq \operatorname{play}(\beta)$, being $\mathrm{p} \neq \operatorname{play}\left(\beta^{\prime}\right)$ for all $\beta^{\prime} \in \Delta$, there is in $\xi$ a first occurrence of $\beta_{0}$ with play $\left(\beta_{0}\right)=\mathrm{p}$, thus we get $\operatorname{depth}(\xi, \mathrm{p})<\operatorname{depth}(\beta \cdot \xi, \mathrm{p})$. This implies $\operatorname{depth}(\widehat{\mathrm{G}}, \mathrm{p})<\operatorname{depth}(\mathrm{G}, \mathrm{p})$.

In both cases we have $\mathrm{G}\|\mathcal{M} \stackrel{\beta}{\rightarrow} \widehat{\mathrm{G}}\| \widehat{\mathcal{M}}$ with $\mathrm{p} \neq \operatorname{play}(\beta)$ and $\operatorname{depth}(\widehat{\mathrm{G}}, \mathrm{p})<\operatorname{depth}(\mathrm{G}, \mathrm{p})$. If $\Delta=\{\beta\}$ we are done. Otherwise we set $\Delta \backslash\{\beta\}=\left\{\beta_{1}, \ldots, \beta_{n}\right\}$, where $n \geq 1$, and we get

$$
\widehat{\mathrm{G}}\left\|\widehat{\mathcal{M}} \stackrel{\beta_{1}}{\longrightarrow} \mathrm{G}_{1}^{\prime}\right\| \mathcal{M}_{1} \stackrel{\beta_{2}}{\longrightarrow} \ldots \stackrel{\beta_{n}}{\longrightarrow} \mathrm{G}_{n}^{\prime} \| \mathcal{M}_{n}
$$

with $\mathrm{G}_{n}^{\prime}=\mathrm{G}^{\prime}$ and $\mathcal{M}_{n}=\mathcal{M}^{\prime}$. By Item (2), we get $\operatorname{depth}\left(\mathrm{G}_{k}^{\prime}, \mathrm{p}\right) \leq \operatorname{depth}\left(\mathrm{G}_{k-1}^{\prime}, \mathrm{p}\right)$ for all $k \in$ 2..n and $\operatorname{depth}\left(\mathrm{G}_{1}^{\prime}, \mathrm{p}\right) \leq \operatorname{depth}(\widehat{\mathrm{G}}, \mathrm{p})$ and this implies $\operatorname{depth}\left(\mathrm{G}^{\prime}, \mathrm{p}\right) \leq \operatorname{depth}(\widehat{\mathrm{G}}, \mathrm{p})<\operatorname{depth}(\mathrm{G}, \mathrm{p})$ as needed.

Theorem 4.12 (No locked inputs). If $\mathrm{G} \vdash \mathbb{N}$ and $\mathrm{G}$ is bounded and $\vdash_{\mathrm{wb}} \mathrm{G} \| \mathcal{M}$, then $\mathbb{N} \| \mathcal{M}$ is input-enabling.

Proof. If $\mathbb{N} \equiv \mathrm{p} \llbracket \mathrm{q} ?\left\{\lambda_{i} ; P_{i}\right\}_{i \in I} \rrbracket \| \mathbb{N}_{0}$, then $\mathrm{G} \vdash \mathbb{N}$ implies $\mathrm{p} \in$ players $(\mathrm{G})$. By Strong Subject Reduction (Theorem 4.9) all complete lockstep computations of $\mathbb{N} \| \mathcal{M}$ are complete lockstep computations of $\mathrm{G} \| \mathcal{M}$. Then it is enough to show that $\mathrm{G} \| \mathcal{M}$ is input-enabling.

We prove the statement by induction on $\operatorname{depth}(\mathrm{G}, \mathrm{p})$. Consider a complete lockstep computation $\left\{\mathrm{G}_{k}\left\|\mathcal{M}_{k} \stackrel{\Delta_{k}}{\underset{k}{\rightleftarrows}} \mathrm{G}_{k+1}\right\| \mathcal{M}_{k+1}\right\}_{k<x}$ with $\mathrm{G}_{0}\left\|\mathcal{M}_{0}=\mathrm{G}\right\| \mathcal{M}$. If $\operatorname{depth}(\mathrm{G}, \mathrm{p})=1$ by Lemma $4.11(1)$ we get $\mathrm{G}\left\|\mathcal{M} \stackrel{\beta}{\rightarrow} \mathrm{G}^{\prime}\right\| \mathcal{M}^{\prime}$, with play $(\beta)=\mathrm{p}$, hence, since $\Delta_{0}$ is a maximal coherent set, there is $\beta^{\prime} \in \Delta_{0}$ such that play $\left(\beta^{\prime}\right)=\operatorname{play}(\beta)=\mathrm{p}$, as needed. If $\operatorname{depth}(\mathrm{G}, \mathrm{p})>1$, let us assume $\mathrm{p} \neq \operatorname{play}(\beta)$ for all $\beta \in \Delta_{0}$ (otherwise the statement is trivial), then, since $\mathrm{G}\left\|\mathcal{M}=\mathrm{G}_{0}\right\| \mathcal{M}_{0} \stackrel{\Delta_{0}}{\Longrightarrow} \mathrm{G}_{1} \| \mathcal{M}_{1}$, by Lemma $4.11(3)$, we $g e t \operatorname{depth}\left(\mathrm{G}_{1}, \mathrm{p}\right)<\operatorname{depth}(\mathrm{G}, \mathrm{p})$. Hence, the statement follows by induction hypothesis.

To avoid messages waiting forever in the queue we need to strengthen weak balancing with the requirement that all messages in the queue will be eventually read. This last restriction is enforced by the auxiliary judgment $\vdash_{\text {read }}(G, \mathcal{M})$, which means that (each path of) $\mathrm{G}$ reads all the messages in $\mathcal{M}$. The inductive definition of this judgment is given at the bottom of Figure 11. If the queue is empty, Rule [EMPTY-R], then the judgement holds. If $\mathrm{G}$ is a choice of outputs, Rule [OUT-R], then in each branch of the choice all the messages in the queue must be read. For an input type $\mathrm{pq} ?\left\{\lambda_{i} ; \mathrm{G}_{i}\right\}_{i \in I}$, if the message at the top of the queue (considered modulo $\equiv$ ) is $\left\langle\mathrm{p}, \lambda_{h}, \mathrm{q}\right\rangle$ for some $h \in I$ we read it, Rule [IN-R1], otherwise we do not to read messages, Rule [IN-R2]. In Rule [IN-R1] we could check only $\vdash_{\text {read }}\left(G_{h}, \mathcal{M}\right)$ since the other branches are discharged. Instead we required $\vdash_{\text {read }}\left(\mathrm{G}_{i}, \mathcal{M}\right)$ for all $i \in I$. This is needed to ensure that a message on top of a queue in a balanced type configuration has a finite weight for the global type (Definition 4.15), see Example 4.17.

Example 4.13. Figure 10 displays the initial part of the weak balancing derivation for the type configuration $G \| \emptyset$, where $G, G_{1}$ and $G_{2}$ are defined in the caption of Figure 4 . We can easily get a balancing derivation by checking the readability side-conditions. Figure 12 only shows the more interesting case.

Comparing Figures 9 and 11 we can see that the balancing is obtained from the weak balancing just by adding the readability judgment as side condition of rules [B-OUT] and [B-IN], checking that all messages in the queue can be read, even in case of infinite derivations, 


$$
\begin{aligned}
& \text { [B-End] } \overline{\overline{\vdash_{\mathrm{b}} \text { End } \| \emptyset}} \\
& \text { [B-Out] } \frac{\vdash_{\mathrm{b}} \mathrm{G}_{i} \| \mathcal{M} \cdot\left\langle\mathrm{p}, \lambda_{i}, \mathrm{q}\right\rangle \quad \forall i \in I}{\vdash_{\mathrm{b}} \mathrm{p} \mathrm{q} !\left\{\lambda_{i} ; \mathrm{G}_{i}\right\}_{i \in I} \| \mathcal{M}} \vdash_{\text {read }}\left(\mathrm{G}_{i}, \mathcal{M}\right) \forall i \in I \\
& {[\mathrm{~B}-\mathrm{IN}] \frac{\vdash_{\mathrm{b}} \mathrm{G}_{h} \| \mathcal{M}}{\vdash_{\mathrm{b}} \mathrm{pq} ?\left\{\lambda_{i} ; \mathrm{G}_{i}\right\}_{i \in I} \|\left\langle\mathrm{p}, \lambda_{h}, \mathrm{q}\right\rangle \cdot \mathcal{M}} \vdash_{\text {read }}\left(\mathrm{G}_{i}, \mathcal{M}\right) \forall i \in I} \\
& \text { [EMPTY-R] } \frac{}{\vdash_{\text {read }}(\mathrm{G}, \emptyset)} \quad[\text { OUT-R }] \frac{\vdash_{\text {read }}\left(\mathrm{G}_{i}, \mathcal{M}\right) \forall i \in I}{\vdash_{\text {read }}\left(\mathrm{pq} !\left\{\lambda_{i} ; \mathrm{G}_{i}\right\}_{i \in I}, \mathcal{M}\right)} \\
& {[\mathrm{IN}-\mathrm{R} 1] \frac{\vdash_{\text {read }}\left(\mathrm{G}_{i}, \mathcal{M}\right) \forall i \in I}{\vdash_{\text {read }}\left(\mathrm{pq} ?\left\{\lambda_{i} ; \mathrm{G}_{i}\right\}_{i \in I},\left\langle\mathrm{p}, \lambda_{h}, \mathrm{q}\right\rangle \cdot \mathcal{M}\right)} \quad h \in I} \\
& {[\mathrm{IN}-\mathrm{R} 2] \frac{\vdash_{\text {read }}\left(\mathrm{G}_{i}, \mathcal{M}\right) \forall i \in I}{\vdash_{\text {read }}\left(\mathrm{pq} ?\left\{\lambda_{i} ; \mathrm{G}_{i}\right\}_{i \in I}, \mathcal{M}\right)} \quad \mathcal{M} \not \equiv\left\langle\mathrm{p}, \lambda_{i}, \mathrm{q}\right\rangle \cdot \mathcal{M}^{\prime} \forall i \in I}
\end{aligned}
$$

Figure 11: Balancing of type configurations.

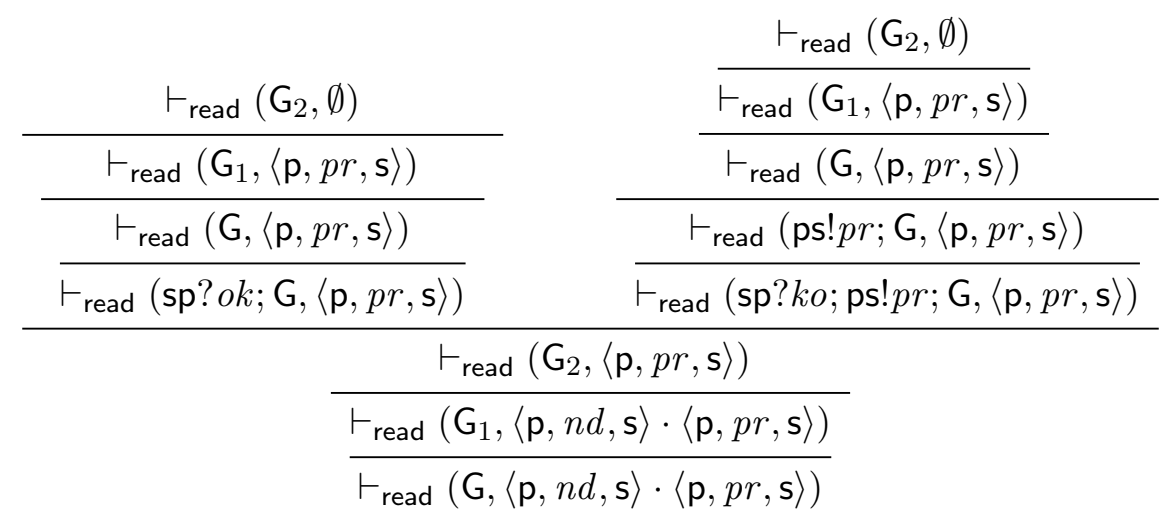

Figure 12: Read of the hospital global type with the queue $\langle\mathrm{p}, n d, \mathrm{~s}\rangle \cdot\langle\mathrm{p}, p r, \mathrm{~s}\rangle$.

see Example 4.14. The necessity of the readability judgment also in Rule [B-IN] is shown in Example 4.17. Notice that this rule by itself only ensures that the first message of the queue is read.

Example 4.14. Let $\mathrm{G}=\mathrm{pq} ! \lambda ; \mathrm{pq} ? \lambda ; \mathrm{G}$ and $\mathcal{M}=\left\langle\mathrm{p}, \lambda^{\prime}, \mathrm{r}\right\rangle$. Then $\mathrm{G} \| \mathcal{M}$ is weakly balanced but not balanced, since $\vdash_{\text {read }}(\mathrm{G}, \mathcal{M})$ does not hold. In fact, $\left\langle\mathrm{p}, \lambda^{\prime}, r\right\rangle$ would never be read.

The regularity of global types and the fact that the initial queue may only decrease ensure the decidability of the readability judgment, while we will show in the next section that both weak balancing and balancing are undecidable.

We use $\mathrm{m}$ as short for a message of the form $\langle p, \lambda, q\rangle$ and let inp $(\langle p, \lambda, q\rangle)=p q$ ? $\lambda$, i.e. $\operatorname{inp}(\langle p, \lambda, q\rangle)$ is the input communication which reads the message $\langle p, \lambda, q\rangle$. We define the weight of a message in a global type, notation $w g(G, m)$, by looking at the corresponding input. 
Definition 4.15 (Weight of messages). For a global type $G$ we define $w g(G, m)$ corecursively as follows: $w g(G, m)=$

$$
\begin{cases}\infty & \text { if } \mathrm{G}=\text { End } \\ 1+\sup _{i \in I} \mathrm{wg}\left(\mathrm{G}_{i}, \mathrm{~m}\right) & \text { if either } \mathrm{G}=\mathrm{pq} !\left\{\lambda_{i} ; \mathrm{G}_{i}\right\}_{i \in I} \\ & \text { or } \mathrm{G}=\mathrm{pq} ?\left\{\lambda_{i} ; \mathrm{G}_{i}\right\}_{i \in I} \text { and } \operatorname{inp}(\mathrm{m}) \neq \mathrm{pq} ? \lambda_{i} \text { for all } i \in I \\ 0 & \text { if } \mathrm{G}=\mathrm{pq} ?\left\{\lambda_{i} ; \mathrm{G}_{i}\right\}_{i \in I} \text { and } \operatorname{inp}(\mathrm{m})=\mathrm{pq} ? \lambda_{h} \text { for some } h \in I\end{cases}
$$

Note that $w g(G, m)$ is well defined as every recursive call is guarded. We get $w g(G, m)=\infty$ when the global type $G$ never reads the message $m$. For example, if $G$ only contains outputs, then $\operatorname{wg}(G, m)=\infty$ for all messages $m$.

The key point is that in a balanced type configuration the message on top of the queue has a finite weight, as proved by the following lemma.

Lemma 4.16. If $\vdash_{\mathrm{b}} \mathrm{G} \| \mathrm{m} \cdot \mathcal{M}$, then $\mathrm{wg}(\mathrm{G}, \mathrm{m})$ is finite.

Proof. By definition $\vdash_{\mathrm{b}} \mathrm{G} \| \mathrm{m} \cdot \mathcal{M}$ implies $\vdash_{\text {read }}(\mathrm{G}, \mathrm{m} \cdot \mathcal{M})$ (see rules in Figure 11). The proof is by induction on the derivation of $\vdash_{\text {read }}(\mathrm{G}, \mathrm{m} \cdot \mathcal{M})$, splitting cases on the last applied rule.

[EMPTY-R]: This is an empty case, because the queue is not empty by hypothesis.

[OUT-R]: We have $\mathrm{G}=\mathrm{pq} !\left\{\lambda_{i} ; \mathrm{G}_{i}\right\}_{i \in I}$ and $\vdash_{\text {read }}\left(\mathrm{G}_{i}, \mathrm{~m} \cdot \mathcal{M}\right)$ for all $i \in I$. By induction hypothesis we get that $\operatorname{wg}\left(\mathrm{G}_{i}, \mathrm{~m}\right)$ is finite for all $i \in I$, hence $\mathrm{wg}(\mathrm{G}, \mathrm{m})=$ $1+\sup _{i \in I} \mathrm{wg}\left(\mathrm{G}_{i}, \mathrm{~m}\right)$ is finite as well.

[IN-R1]: We have $\mathrm{G}=\mathrm{pq} ?\left\{\lambda_{i} ; \mathrm{G}_{i}\right\}_{i \in I}$ and $\mathrm{m} \cdot \mathcal{M} \equiv\left\langle\mathrm{p}, \lambda_{h}, \mathrm{q}\right\rangle \cdot \mathcal{M}^{\prime}$ with $h \in I$ and $\vdash_{\text {read }}\left(\mathrm{G}_{i}, \mathcal{M}^{\prime}\right)$ for all $i \in I$. If $\mathrm{m}=\left\langle\mathrm{p}, \lambda_{h}, \mathrm{q}\right\rangle$, then $\mathrm{wg}(\mathrm{G}, \mathrm{m})=0$ by definition. Otherwise, $\mathcal{M}^{\prime} \equiv \mathrm{m} \cdot \mathcal{M}^{\prime \prime}$, hence, by induction hypothesis, $\operatorname{wg}\left(\mathrm{G}_{i}, \mathrm{~m}\right)$ is finite for all $i \in I$ and $\operatorname{so} \mathrm{wg}(\mathrm{G}, \mathrm{m})=1+\sup _{i \in I} \mathrm{wg}\left(\mathrm{G}_{i}, \mathrm{~m}\right)$ is finite as well.

[IN-R2]: We have $\mathrm{G}=\mathrm{pq} ?\left\{\lambda_{i} ; \mathrm{G}_{i}\right\}_{i \in I}$ and $\mathrm{m} \cdot \mathcal{M} \not \equiv\left\langle\mathrm{p}, \lambda_{i}, \mathrm{q}\right\rangle \cdot \mathcal{M}^{\prime}$ and $\vdash_{\text {read }}\left(\mathrm{G}_{i}, \mathrm{~m} \cdot \mathcal{M}\right)$ for all $i \in I$. Therefore, $\mathrm{m} \neq\left\langle\mathrm{p}, \lambda_{i}, \mathrm{q}\right\rangle$ for all $i \in I$ and, by induction hypothesis, $\operatorname{wg}\left(\mathrm{G}_{i}, \mathrm{~m}\right)$ is finite for all $i \in I$, hence, $\mathrm{wg}(\mathrm{G}, \mathrm{m})=1+\sup _{i \in I} \mathrm{wg}\left(\mathrm{G}_{i}, \mathrm{~m}\right)$ is finite as well.

Example 4.17. Lemma 4.16 fails if either Rule [B-IN] does not require the readability judgment as side condition or in the premise of Rule [IN-R1] we only consider the global type $\mathrm{G}_{h}$. A simple example is $\mathrm{G}=\mathrm{pq}$ ? $\left\{\lambda_{1} ; \mathrm{pr} ? \lambda_{2}, \lambda_{3}\right\}$ and $\mathcal{M}=\left\langle\mathrm{p}, \lambda_{1}, \mathrm{q}\right\rangle \cdot\left\langle\mathrm{p}, \lambda_{2}, \mathrm{r}\right\rangle$. In fact, in both cases we would get $\vdash_{\mathrm{b}} \mathrm{G} \| \mathcal{M}$ (derived from $\vdash_{\text {read }}(\mathrm{G}, \mathcal{M})$ in the second case), but $w g\left(G,\left\langle p, \lambda_{2}, r\right\rangle\right)=\infty$.

A last lemma before establishing the property of "No orphan message" relates the weight of messages in global types with the reductions of the corresponding type configurations.

Lemma 4.18. Let $\vdash_{\mathrm{b}} \mathrm{G} \| \mathcal{M}$ and $\mathcal{M} \equiv \mathrm{m} \cdot \mathcal{M}^{\prime}$, then the following hold:

(1) If $\mathrm{wg}(\mathrm{G}, \mathrm{m})=0$, then $\mathrm{G}\left\|\mathcal{M} \stackrel{\operatorname{inp}(\mathrm{m})}{\longrightarrow} \mathrm{G}^{\prime}\right\| \mathcal{M}^{\prime}$.

(2) If $\mathrm{G}\|\mathcal{M} \stackrel{\beta}{\rightarrow} \widehat{\mathrm{G}}\| \widehat{\mathcal{M}}$ with $\operatorname{inp}(\mathrm{m}) \neq \beta$, then $\mathrm{wg}(\widehat{\mathrm{G}}, \mathrm{m}) \leq \mathrm{wg}(\mathrm{G}, \mathrm{m})$ and $\widehat{\mathcal{M}} \equiv \mathrm{m} \cdot \widehat{\mathcal{M}}^{\prime}$.

(3) If $\mathrm{G}\|\mathcal{M} \triangleq \widehat{\mathrm{G}}\| \widehat{\mathcal{M}}$, with $\operatorname{inp}(\mathrm{m}) \notin \Delta$, then $\operatorname{wg}(\widehat{\mathrm{G}}, \mathrm{m})<\operatorname{wg}(\mathrm{G}, \mathrm{m})$ and $\widehat{\mathcal{M}} \equiv \mathrm{m} \cdot \widehat{\mathcal{M}}^{\prime}$.

Proof. (1). Assume $\mathrm{m}=\langle\mathrm{p}, \lambda, \mathrm{q}\rangle$, hence $\operatorname{inp}(\mathrm{m})=\mathrm{pq}$ ? $\lambda$. If $\operatorname{wg}(\mathrm{G}, \mathrm{m})=0$, then $\mathrm{G}=$ $\mathrm{pq} ?\left\{\lambda_{i} ; \mathrm{G}_{i}\right\}_{i \in I}$ and $\lambda=\lambda_{h}$ and by Rule [Top-IN] we have $\mathrm{G}\left\|\mathcal{M} \stackrel{\mathrm{pq} ? \lambda}{\longrightarrow} \mathrm{G}_{h}\right\| \mathcal{M}^{\prime}$ for some $h \in I$. 
(2). The fact that $\widehat{\mathcal{M}} \equiv \mathrm{m} \cdot \widehat{\mathcal{M}}^{\prime}$ is immediate, since $\operatorname{inp}(\mathrm{m}) \neq \beta$. We prove $\operatorname{wg}(\widehat{\mathrm{G}}, \mathrm{m}) \leq$ $w g(G, m)$ by induction on reduction rules for type configurations.

[Top-OUT]: Then $\mathrm{G}=\mathrm{pq} !\left\{\lambda_{i} ; \mathrm{G}_{i}\right\}_{i \in I}$ and $\widehat{\mathcal{M}}=\mathcal{M} \cdot\left\langle\mathrm{p}, \lambda_{h}, \mathrm{q}\right\rangle$ and $\widehat{\mathrm{G}}=\mathrm{G}_{h}$ for some $h \in I$. By Definition $4.15 \mathrm{wg}(\mathrm{G}, \mathrm{m})=1+\sup _{i \in I} \mathrm{wg}\left(\mathrm{G}_{i}, \mathrm{~m}\right)>\mathrm{wg}\left(\mathrm{G}_{h}, \mathrm{~m}\right)$.

[Top-IN]: Then $\mathrm{G}=\mathrm{pq} ?\left\{\lambda_{i} ; \mathrm{G}_{i}\right\}_{i \in I}$ and $\mathcal{M}=\left\langle\mathrm{p}, \lambda_{h}, \mathrm{q}\right\rangle \cdot \widehat{\mathcal{M}}$ and $\widehat{\mathrm{G}}=\mathrm{G}_{h}$ for some $h \in I$. By Definition $4.15 \mathrm{wg}(\mathrm{G}, \mathrm{m})=1+\sup _{i \in I} \mathrm{wg}\left(\mathrm{G}_{i}, \mathrm{~m}\right)>\mathrm{wg}\left(\mathrm{G}_{h}, \mathrm{~m}\right)$.

[INSIDE-OUT]: Then $\mathrm{G}=\mathrm{pq} !\left\{\lambda_{i} ; \mathrm{G}_{i}\right\}_{i \in I}$ and $\widehat{\mathrm{G}}=\mathrm{pq} !\left\{\lambda_{i} ; \mathrm{G}_{i}^{\prime}\right\}_{i \in I}$ and $\mathrm{G}_{i} \| \mathcal{M} \cdot\left\langle\mathrm{p}, \lambda_{i}, \mathrm{q}\right\rangle \stackrel{\beta}{\rightarrow}$ $\mathrm{G}_{i}^{\prime} \| \widehat{\mathcal{M}} \cdot\left\langle\mathrm{p}, \lambda_{i}, \mathrm{q}\right\rangle$ for all $i \in I$. By Definition $4.15 \mathrm{wg}(\mathrm{G}, \mathrm{m})=1+\sup _{i \in I} \mathrm{wg}\left(\mathrm{G}_{i}, \mathrm{~m}\right)$ and $\mathrm{wg}(\widehat{\mathrm{G}}, \mathrm{m})=1+\sup _{i \in I} \mathrm{wg}\left(\mathrm{G}_{i}^{\prime}, \mathrm{m}\right)$. By induction $\mathrm{wg}\left(\mathrm{G}_{i}^{\prime}, \mathrm{m}\right) \leq \mathrm{wg}\left(\mathrm{G}_{i}, \mathrm{~m}\right)$ for all $i \in I$ and this concludes the proof.

[INsIDE-IN]: Then $\mathrm{G}=\mathrm{pq} ?\left\{\lambda_{i} ; \mathrm{G}_{i}\right\}_{i \in I}$ and $\widehat{\mathrm{G}}=\mathrm{pq} ?\left\{\lambda_{i} ; \mathrm{G}_{i}^{\prime}\right\}_{i \in I}$ and $\mathcal{M} \equiv\left\langle\mathrm{p}, \lambda_{k}, \mathrm{q}\right\rangle \cdot \mathcal{M}^{\prime \prime}$ and $\widehat{\mathcal{M}} \equiv\left\langle\mathrm{p}, \lambda_{k}, \mathrm{q}\right\rangle \cdot \widehat{\mathcal{M}}^{\prime}$ for some $k \in I$ and $\mathrm{G}_{i}\left\|\mathcal{M}^{\prime \prime} \stackrel{\beta}{\rightarrow} \mathrm{G}_{i}^{\prime}\right\| \widehat{\mathcal{M}}^{\prime}$ for all $i \in I$. If $\mathrm{m}=$ $\left\langle\mathrm{p}, \lambda_{k}, \mathrm{q}\right\rangle$, then $\mathrm{wg}(\mathrm{G}, \mathrm{m})=\mathrm{wg}(\widehat{\mathrm{G}}, \mathrm{m})=0$. Otherwise $w g(\mathrm{G}, \mathrm{m})=1+\sup _{i \in I} \mathrm{wg}\left(\mathrm{G}_{i}, \mathrm{~m}\right)$ and $w g(\widehat{G}, \mathrm{~m})=1+\sup _{i \in I} w g\left(\mathrm{G}_{i}^{\prime}, \mathrm{m}\right)$. By induction $w g\left(\mathrm{G}_{i}^{\prime}, \mathrm{m}\right) \leq \mathrm{wg}\left(\mathrm{G}_{i}, \mathrm{~m}\right)$ for all $i \in I$ and this concludes the proof.

(3). Let $\Delta=\left\{\beta_{1}, \ldots, \beta_{n}\right\}$, hence by definition of lockstep transition we have

$$
\mathrm{G}_{k-1}\left\|\mathcal{M}_{k-1} \stackrel{\beta_{k}}{\longrightarrow} \mathrm{G}_{k}\right\| \mathcal{M}_{k}
$$

for all $k \in 1$..n, with $\mathrm{G}\left\|\mathcal{M}=\mathrm{G}_{0}\right\| \mathcal{M}_{0}$ and $\widehat{\mathrm{G}}\left\|\widehat{\mathcal{M}}=\mathrm{G}_{n}\right\| \mathcal{M}_{n}$. By Item (2), for all $k \in 1 . . n$, we have $\mathrm{wg}\left(\mathrm{G}_{k}, \mathrm{~m}\right) \leq \mathrm{wg}\left(\mathrm{G}_{k-1}, \mathrm{~m}\right)$ and $\mathcal{M}_{k} \equiv \mathrm{m} \cdot \mathcal{M}_{k}^{\prime}$, hence $\widehat{\mathcal{M}} \equiv \mathrm{m} \cdot \widehat{\mathcal{M}}^{\prime}$. We have two cases on $\mathrm{G}$.

- If $\mathrm{G}=\mathrm{pq} !\left\{\lambda_{i} ; \mathrm{G}_{i}^{\prime}\right\}_{i \in I}$, as $\Delta$ is maximal, we have $\beta_{j}=\mathrm{pq} ! \lambda_{h}$ for some $j \in 1$..n and $h \in I$, and so $\mathrm{G}_{j-1}=\mathrm{pq} !\left\{\lambda_{i} ; \mathrm{G}_{i}^{\prime \prime}\right\}_{i \in I}$ and the step $\mathrm{G}_{j-1}\left\|\mathcal{M}_{j-1} \stackrel{\beta_{j}}{\longrightarrow} \mathrm{G}_{j}\right\| \mathcal{M}_{j}$ is derived by Rule [Top-OUT], hence $\mathrm{G}_{j}=\mathrm{G}_{h}^{\prime \prime}$. Then, since $\mathrm{wg}\left(\mathrm{G}_{j-1}, \mathrm{~m}\right)=1+\sup _{i \in I} \mathrm{wg}\left(\mathrm{G}_{i}^{\prime \prime}, \mathrm{m}\right)$ and it is finite, we get $w g\left(G_{j}, m\right)<w g\left(G_{j-1}, m\right)$ and this proves the statement.

- If $\mathrm{G}=\mathrm{pq}$ ? $\left\{\lambda_{i} ; \mathrm{G}_{i}^{\prime}\right\}_{i \in I}$, as $\vdash_{\mathrm{b}} \mathrm{G} \| \mathrm{m} \cdot \mathcal{M}$ holds, we have $\mathrm{m} \cdot \mathcal{M} \equiv\left\langle\mathrm{p}, \lambda_{h}, \mathrm{q}\right\rangle \cdot \mathcal{M}^{\prime}$ for some $h \in I$. Since $\Delta$ is maximal, we have $\beta_{j}=\mathrm{pq} ? \lambda_{h}$ for some $j \in 1 . . n$ and so $\mathrm{G}_{j-1}=\mathrm{pq} ?\left\{\lambda_{i} ; \mathrm{G}_{i}^{\prime \prime}\right\}_{i \in I}$ and the step $\mathrm{G}_{j-1}\left\|\mathcal{M}_{j-1} \stackrel{\beta_{j}}{\longrightarrow} \mathrm{G}_{j}\right\| \mathcal{M}_{j}$ is derived by Rule [ToP-IN], hence $\mathrm{G}_{j}=\mathrm{G}_{h}^{\prime \prime}$. Then, since $\operatorname{wg}\left(\mathrm{G}_{j-1}, \mathrm{~m}\right)=1+\sup _{i \in I} \mathrm{wg}\left(\mathrm{G}_{i}^{\prime \prime}, \mathrm{m}\right)$ and it is finite, we get $\mathrm{wg}\left(\mathrm{G}_{j}, \mathrm{~m}\right)<\mathrm{wg}\left(\mathrm{G}_{j-1}, \mathrm{~m}\right)$ and this proves the statement.

Theorem 4.19 (No orphan messages). If $\mathrm{G} \vdash \mathbb{N}$ and $\mathrm{G}$ is bounded and $\vdash_{\mathrm{b}} \mathrm{G} \| \mathcal{M}$, then $\mathbb{N} \| \mathcal{M}$ is queue-consuming.

Proof. By Strong Subject Reduction (Theorem 4.9) all complete lockstep computations of $\mathbb{N} \| \mathcal{M}$ are complete lockstep computations of $\mathrm{G} \| \mathcal{M}$. Then it is enough to show that $\mathrm{G} \| \mathcal{M}$ is queue-consuming.

We prove the statement by induction on $\operatorname{wg}(\mathrm{G}, \mathrm{m})$. Consider a complete lockstep computation $\left\{\mathrm{G}_{k}\left\|\mathcal{M}_{k} \stackrel{\Delta_{k}}{\underset{k}{\rightleftarrows}} \mathrm{G}_{k+1}\right\| \mathcal{M}_{k+1}\right\}_{k<x}$ where $\mathrm{G}_{0}\left\|\mathcal{M}_{0}=\mathrm{G}\right\| \mathcal{M}$ with $\mathcal{M} \equiv \mathrm{m} \cdot \widehat{\mathcal{M}}$. If $w g(G, m)=0$, by Lemma $4.18(1)$ we get $G\left\|\mathcal{M} \stackrel{\operatorname{inp}(m)}{\longrightarrow} G^{\prime}\right\| \mathcal{M}^{\prime}$ with $\mathcal{M}^{\prime} \equiv \widehat{\mathcal{M}}$, then, since $\Delta_{0}$ is a maximal coherent set, we have $\operatorname{inp}(\mathrm{m}) \in \Delta_{0}$ as needed. If $\operatorname{wg}(\mathrm{G}, \mathrm{m})>0$, let us assume $\operatorname{inp}(\mathrm{m}) \notin \Delta_{0}$ (otherwise the statement is trivial), then by Lemma $4.18(3)$ 
$\mathrm{G}\left\|\mathcal{M} \stackrel{\Delta_{0}}{\Longrightarrow} \mathrm{G}_{1}\right\| \mathcal{M}_{1}$ implies $\mathcal{M}_{1} \equiv \mathrm{m} \cdot \mathcal{M}_{1}^{\prime}$ and $\mathrm{wg}\left(\mathrm{G}_{1}, \mathrm{~m}\right)<\mathrm{wg}(\mathrm{G}, \mathrm{m})$. Hence the statement follows by induction hypothesis.

Now progress is an immediate consequence of Theorems 4.8, 4.12 and 4.19.

Theorem 4.20 (Progress). If $\mathrm{G} \vdash \mathbb{N}$ and $\mathrm{G}$ is bounded and $\vdash_{\mathrm{b}} \mathrm{G} \| \mathcal{M}$, then $\mathbb{N} \| \mathcal{M}$ has the progress property.

\section{The Undecidability of (WEAK) BALANCING}

The cornerstone we build on to prove well-typed sessions have progress is the balancing property. This has a non-trivial definition mixing coinduction and induction, hence a natural question which may arise is whether it is decidable.

In this section we answer this question, proving that (weak) balancing is actually undecidable. Then, in the next section, we will define an algorithm to test balancing of a type configuration proving it is sound with respect to the coinductive definition. We carry out these results for the balancing property and only discuss how they can be easily adapted to the weak version.

The undecidability proof follows the same strategy used to show the undecidability of asynchronous session subtyping [BCZ17]. Indeed, we provide a reduction from the complement of the acceptance problem on queue machines, known to be undecidable as queue machines are Turing complete [Koz97], to the problem of checking if a given type configuration has the balancing property. We first recall basic definitions about queue machines.

Definition 5.1 (Queue machine). A queue machine is a tuple $M=\langle Q, \Sigma, \Gamma, \$, s, \delta\rangle$ where

- $Q$ is a finite set of states;

- $\Gamma$ is a finite set named queue alphabet;

- $\Sigma \subseteq \Gamma$ is a finite set named input alphabet;

- $\$ \in \Gamma \backslash \Sigma$ is the initial queue symbol;

- $s \in Q$ is the initial state;

- $\delta: Q \times \Gamma \rightarrow Q \times \Gamma^{\star}$ is the transition function.

A configuration of $M$ is a pair $\langle q, \chi\rangle \in Q \times \Gamma^{\star}$, where $q$ is the current state and $\chi$ is a sequence of symbols in $\Gamma$ modelling the queue. An initial configuration has shape $\langle s, \chi \$\rangle$ where $\chi \in \Sigma^{\star}$.

The transition relation $\rightarrow_{M}$ is a binary relation on configurations of $M$ defined by

$$
\langle q, a \alpha\rangle \rightarrow_{M}\left\langle q^{\prime}, \alpha \eta\right\rangle \quad \text { if } \quad \delta(q, a)=\left\langle q^{\prime}, \eta\right\rangle
$$

Note that, since $\delta$ is a total function, computation is deterministic and the only final configurations of the machine $M$ have shape $\langle q, \epsilon\rangle$. That is, $M$ terminates only when the queue is empty.

We can now define the acceptance condition for a queue machine. As usual, we denote by $\rightarrow_{M}^{\star}$ the reflexive and transitive closure of the transition relation.

Definition 5.2 (Acceptance). Let $M=\langle Q, \Sigma, \Gamma, \$, s, \delta\rangle$ be a queue machine. A sequence $\chi \in \Sigma^{\star}$ is accepted iff $\langle s, \chi \$\rangle \rightarrow_{M}^{\star}\langle q, \epsilon\rangle$, for some $q \in Q$.

Intuitively, this means that a sequence $\chi$ on the input alphabet is accepted iff starting from $\langle s, \chi \$\rangle$ the machine terminates. 
Let us now define the encoding which will give us the reduction. Assume a queue machine $M=(Q, \Sigma, \Gamma, \$, s, \delta)$. We define a global type whose players are $\mathrm{p}$ and $\mathrm{q}$ and labels are symbols in $\Gamma$. Since $p$ is always the sender and $q$ the receiver in defining the global type we avoid prefixing! and ? by pq. Moreover in the queue we only write the exchanged labels, that is, we identify message queues (in type configurations) with elements of $\Gamma^{\star}$. For every state $q \in Q$, let $\mathrm{G}_{q}$ be defined by the following equation:

$$
\mathrm{G}_{q}=?\left\{a ; ! b_{1}^{a} ; \ldots ; ! b_{n_{a}}^{a} ; \mathrm{G}_{q^{\prime}}\right\}_{a \in \Gamma} \quad \text { if } \delta(q, a)=\left(q^{\prime}, b_{1}^{a} \ldots b_{n_{a}}^{a}\right)
$$

I.e. the global type $\mathrm{G}_{q}$ is an input reading all characters $a$ of the queue alphabet followed by the outputs of the characters $b_{1}^{a}, \ldots, b_{n_{a}}^{a}$ which are put on the queue and then by the global type $\mathrm{G}_{q^{\prime}}$, where $q^{\prime}$ is the following state.

The encoding of the machine configuration $\langle q, \chi\rangle$, denoted by $[\langle q, \chi\rangle]$, is the type configuration $\mathrm{G}_{q} \| \chi$.

Our goal is to relate the acceptance problem of the queue machine $M$ with the balancing property of the type configurations obtained through the above encoding. The next theorem formally states this relationship.

Theorem 5.3. The machine $M$ does not accept $\chi \in \Sigma^{\star}$ iff the encoding of its initial configuration $\langle s, \chi \$\rangle$ is balanced, that is, $\vdash_{\mathrm{b}} \mathrm{G}_{s} \| \chi \$$.

To prove the theorem we rely on some lemmas. The first one shows that diverging computations in a queue machine correspond to derivations of the balancing property.

Lemma 5.4. If $\langle q, \chi\rangle$ diverges, then $\vdash_{\mathrm{b}} \mathrm{G}_{q} \| \chi$ holds.

Proof. Since $\langle q, \chi\rangle$ diverges, we have a sequence $\left\langle q_{i}, \chi_{i}\right\rangle_{i \in \mathbb{N}}$ such that $\langle q, \chi\rangle=\left\langle q_{0}, \chi_{0}\right\rangle$ and, for all $i \in \mathbb{N},\left\langle q_{i}, \chi_{i}\right\rangle \rightarrow_{M}\left\langle q_{i+1}, \chi_{i+1}\right\rangle$. By definition of $\rightarrow_{M}$ we have $\chi_{i}=a_{i} \eta_{i}$ and $\delta\left(q_{i}, a_{i}\right)=\left\langle q_{i+1}, b_{1}^{a_{i}} \ldots b_{n_{a_{i}}}^{a_{i}}\right\rangle$. For all $i \in \mathbb{N}$, define the set $A_{i}$ of balancing judgments as follows:

$$
A_{i}=\left\{\vdash_{\mathrm{b}} \mathrm{G}_{q_{i}}\left\|\chi_{i}, \vdash_{\mathrm{b}} ! b_{1}^{a_{i}} ; \ldots ! b_{n_{a_{i}}}^{a_{i}} ; \mathrm{G}_{q_{i+1}}\right\| \eta_{i}, \ldots, \vdash_{\mathrm{b}} ! b_{n_{a_{i}}}^{a_{i}} ; \mathrm{G}_{q_{i+1}} \| \eta_{i} b_{1}^{a_{i}} \ldots b_{n_{a_{i}-1}}^{a_{i}}\right\}
$$

and let $A=\bigcup_{i \in \mathbb{N}} A_{i}$. We prove that $A$ is consistent with respect to the rules defining balancing, then the statement follows by coinduction.

We can easily prove that for every judgement $\vdash_{\mathrm{b}} \mathrm{G} \| \eta$ in $A$, the judgement $\vdash_{\text {read }}(\mathrm{G}, \eta)$ is derivable. In fact each $\eta$ only contains symbols in $\Gamma$ and $\mathrm{G}$ always offers some outputs and then a choice of inputs for all symbols in $\Gamma$.

Therefore, to conclude, we have just to show that every $\vdash_{\mathrm{b}} \mathrm{G} \| \eta$ in $A$ is the consequence of a rule whose premises are still in $A$. By definition of $A$, we have that $\vdash_{\mathrm{b}} \mathrm{G} \| \eta$ belongs to $A_{i}$ for some $i \in \mathbb{N}$. We distinguish three cases.

- If $\mathrm{G}=\mathrm{G}_{q_{i}}$, then $\eta=\chi_{i}=a_{i} \eta_{i}$ and, by definition of $\mathrm{G}_{q_{i}}, \mathrm{G}=$ ? $\left\{a ; \mathrm{G}_{a}\right\}_{a \in \Gamma}$ with $\mathrm{G}_{a_{i}}=$ $! b_{1}^{a_{i}} ; \ldots . ! b_{n_{a_{i}}}^{a_{i}} ; \mathrm{G}_{q_{i+1}}$. Thus, $\vdash_{\mathrm{b}} \mathrm{G} \| \eta$ is the consequence of Rule [B-IN] with unique premise $\vdash_{\mathrm{b}} \mathrm{G}_{a_{i}} \| \eta_{i}$, that belongs to $A_{i} \subseteq A$ by definition.

- If $\mathrm{G}=! b_{k}^{a_{i}} ; \ldots ! b_{n_{a_{i}}}^{a_{i}} ; \mathrm{G}_{q_{i+1}}$ for some $k<n_{a_{i}}$, then $\eta=\eta_{i} b_{1}^{a_{i}} \ldots b_{k-1}^{a_{i}}$. Thus, $\vdash_{\mathrm{b}} \mathrm{G} \| \eta$ is the consequence of Rule [B-OUT] with unique premise $\vdash_{\mathrm{b}} ! b_{k+1}^{a_{i}} ; \ldots ! b_{n_{a_{i}}}^{a_{i}} ; \mathrm{G}_{q_{i+1}} \| \eta b_{k}^{a_{i}}$, that belongs to $A_{i} \subseteq A$ by definition.

- If $\mathrm{G}=! b_{n_{a_{i}}}^{a_{i}} ; \mathrm{G}_{q_{i+1}}$, then $\eta=\eta_{i} b_{1}^{a_{i}} \ldots b_{n_{a_{i}-1}}^{a_{i}}$. Thus, $\vdash_{\mathrm{b}} \mathrm{G} \| \eta$ is the consequence of Rule [B-OUT] with unique premise $\vdash_{\mathrm{b}} \mathrm{G}_{q_{i+1}} \| \eta b_{n_{a_{i}}}^{a_{i}}$ that belongs to $A_{i+1} \subseteq A$ as $\eta b_{n_{a_{i}}}^{a_{i}}=$ $\eta_{i} b_{1}^{a_{i}} \ldots b_{n_{a_{i}}}^{a_{i}}=\chi_{i+1}$ by definition. 
The following lemma shows that transitions in the queue machine preserve the balancing property.

Lemma 5.5. If $\langle q, \chi\rangle \rightarrow_{M}\left\langle q^{\prime}, \chi^{\prime}\right\rangle$ and $\vdash_{\mathrm{b}} \mathrm{G}_{q} \| \chi$, then $\vdash_{\mathrm{b}} \mathrm{G}_{q^{\prime}} \| \chi^{\prime}$.

Proof. If $\langle q, \chi\rangle \rightarrow_{M}\left\langle q^{\prime}, \chi^{\prime}\right\rangle$, then $\chi=a \alpha, \delta(q, a)=\left\langle q^{\prime}, \eta\right\rangle$ and $\chi^{\prime}=\alpha \eta$ with $\eta=b_{1}^{a} \ldots b_{n_{a}}^{a}$. If $\vdash_{\mathrm{b}} \mathrm{G}_{q} \| \chi$ holds, since $\mathrm{G}_{q}=?\left\{a^{\prime} ; b_{1}^{a^{\prime}} ; \ldots b_{n_{a^{\prime}}}^{a^{\prime}} ; \mathrm{G}_{q_{a^{\prime}}}\right\}_{a^{\prime} \in \Gamma}$ with $\delta\left(q, a^{\prime}\right)=\left\langle q_{a^{\prime}}, b_{1}^{a^{\prime}} \ldots b_{n_{a^{\prime}}}^{a^{\prime}}\right\rangle$, we get $q_{a}=q^{\prime}$ and $\vdash_{\mathrm{b}} \mathrm{G}_{q} \| \chi$ is derived by Rule [B-IN] with unique premise $\vdash_{\mathrm{b}} ! b_{1}^{a} ; \ldots b_{n_{a}}^{a} ; \mathrm{G}_{q^{\prime}} \| \alpha$. This premise is obtained by applying $n_{a}$ times Rule [B-OUT] to the judgement $\vdash_{\mathrm{b}} \mathrm{G}_{q^{\prime}} \| \alpha b_{1}^{a} \ldots b_{n_{a}}^{a}$, hence it holds as needed.

Finally, the next lemma shows that a configuration $\langle q, \chi\rangle$ whose encoding has the balancing property is not stuck.

Lemma 5.6. If $\vdash_{\mathrm{b}} \mathrm{G}_{q} \| \chi$ holds, then $\langle q, \chi\rangle \rightarrow_{M}$.

Proof. By definition we have $\mathrm{G}_{q}=?\left\{a ; ! b_{1}^{a} ; \ldots ! b_{n_{a}}^{a} ; \mathrm{G}_{q_{a}}\right\}_{a \in \Gamma}$ with $\delta(q, a)=\left\langle q_{a}, b_{1}^{a} \ldots b_{n_{a}}^{a}\right\rangle$. Then, $\vdash_{\mathrm{b}} \mathrm{G}_{q} \| \chi$ is derived by Rule [B-IN], therefore $\chi=a^{\prime} \chi^{\prime}$ for some $a^{\prime} \in \Gamma$ and $\chi^{\prime} \in \Gamma^{\star}$ and so $\langle q, \chi\rangle \rightarrow_{M}\left\langle q_{a^{\prime}}, \chi^{\prime} b_{1}^{a^{\prime}} \ldots b_{n_{a^{\prime}}}^{a^{\prime}}\right\rangle$.

Proof of Theorem 5.3. To prove the left-to-right implication, note that if $M$ does not accept $\chi \in \Sigma^{\star}$, then $\langle s, \chi \$\rangle$ diverges, hence, by Lemma 5.4, we get $\vdash_{\mathrm{b}} \mathrm{G}_{s} \| \chi \$$. Towards a proof of the other implication, we inductively construct an infinite computations $\left\langle q_{i}, \chi_{i}\right\rangle$ where $\vdash_{\mathrm{b}} \mathrm{G}_{q_{i}} \| \chi_{i}$ holds for all $i \in \mathbb{N}$ as follows:

- set $\left\langle q_{0}, \chi_{0}\right\rangle=\langle s, \chi \$\rangle$, then $\vdash_{\mathrm{b}} \mathrm{G}_{q_{0}} \| \chi_{0}$ holds by hypothesis;

- since $\vdash_{\mathrm{b}} \mathrm{G}_{q_{i}} \| \chi_{i}$ holds by induction hypothesis, by Lemma 5.6, we have $\left\langle q_{i}, \chi_{i}\right\rangle \rightarrow_{M}\left\langle q^{\prime}, \chi^{\prime}\right\rangle$ and, by Lemma 5.5, we get $\vdash_{\mathrm{b}} \mathrm{G}_{q^{\prime}} \| \chi^{\prime}$ holds. Then, we set $\left\langle q_{i+1}, \chi_{i+1}\right\rangle=\left\langle q^{\prime}, \chi^{\prime}\right\rangle$.

Therefore, $\langle s, \chi \$\rangle$ diverges and so $\chi$ is not accepted.

Corollary 5.7. The balancing property is undecidable.

We can get the same result for weak balancing just removing the parts of the proofs dealing with the readability judgement in Lemmas 5.4, 5.5, as they do not play any relevant role in the reduction.

\section{RECOVERING EFFECTIVENESS}

In order to recover from the undecidability result in Corollary 5.7, we define an inductive version of the balancing property, proving it is sound with respect to the coinductive definition (Theorem 6.8), thus getting a sound algorithm to check balancing. Note that this soundness result is enough to ensure that well-typed sessions - where the corresponding type configuration is successfully checked by this algorithm - has progress, thus obtaining an effective type system.

This inductive definition, described by the rules in Figure 13, follows a standard pattern used to deal with regular structures: we consider an enriched judgement $\mathcal{H} \vdash_{\mathrm{b}}^{\mathcal{I}} \mathrm{G} \| \mathcal{M}$, where $\mathcal{H}$ is an auxiliary set of pairs of types, queues used to detect cycles in global types and test a condition on them (see the side condition of Rule [IB-CYCLE]), thus avoiding non-termination. Other rules are essentially the same as in the coinductive version.

To make this pattern work, we have to appropriately define the judgement $\vdash_{\text {ok }}\left(\mathrm{G}, \mathcal{M}, \mathcal{M}^{\prime}\right)$. One obvious possibility is to require $\mathcal{M} \equiv \mathcal{M}^{\prime}$ and $\vdash_{\text {read }}(\mathrm{G}, \mathcal{M})$, which is always sound, because it forces derivations to be regular. However, this is too restrictive, as it accepts 


$$
\begin{aligned}
& \text { [IB-End] } \overline{\mathcal{H} \vdash_{\mathrm{b}}^{\mathcal{I}} \text { End } \| \emptyset} \quad[\text { IB-CyCLE }] \overline{\mathcal{H},(\mathrm{G}, \mathcal{M}) \vdash_{\mathrm{b}}^{\mathcal{I}} \mathrm{G} \| \mathcal{M}^{\prime}} \vdash_{\text {ok }}\left(\mathrm{G}, \mathcal{M}, \mathcal{M}^{\prime}\right) \\
& \text { [IB-OUT] } \frac{\mathcal{H},\left(\mathrm{p} \mathrm{q} !\left\{\lambda_{i} ; \mathrm{G}_{i}\right\}_{i \in I}, \mathcal{M}\right) \vdash_{\mathrm{b}}^{\mathcal{I}} \mathrm{G}_{i} \| \mathcal{M} \cdot\left\langle\mathrm{p}, \lambda_{i}, \mathrm{q}\right\rangle \quad \forall i \in I}{\mathcal{H} \vdash_{\mathrm{b}}^{\mathcal{I}} \mathrm{pq} !\left\{\lambda_{i} ; \mathrm{G}_{i}\right\}_{i \in I} \| \mathcal{M}} \vdash_{\text {read }}\left(\mathrm{pq} !\left\{\lambda_{i} ; \mathrm{G}_{i}\right\}_{i \in I}, \mathcal{M}\right) \\
& {\left[\text { IB-IN] } \frac{\mathcal{H},\left(\mathrm{p} \mathrm{q} ?\left\{\lambda_{i} ; \mathrm{G}_{i}\right\}_{i \in I},\left\langle\mathrm{p}, \lambda_{h}, \mathrm{q}\right\rangle \cdot \mathcal{M}\right) \vdash_{\mathrm{b}}^{\mathcal{I}} \mathrm{G}_{h} \| \mathcal{M}}{\mathcal{H} \vdash_{\mathrm{b}}^{\mathcal{I}} \mathrm{pq} ?\left\{\lambda_{i} ; \mathrm{G}_{i}\right\}_{i \in I} \|\left\langle\mathrm{p}, \lambda_{h}, \mathrm{q}\right\rangle \cdot \mathcal{M}} \vdash_{\text {read }}\left(\mathrm{pq} ?\left\{\lambda_{i} ; \mathrm{G}_{i}\right\}_{i \in I},\left\langle\mathrm{p}, \lambda_{h}, \mathrm{q}\right\rangle \cdot \mathcal{M}\right) h \in I\right.}
\end{aligned}
$$

Figure 13: Balancing of type configurations (inductive version).

only type configurations where the queue cannot grow indefinitely. This for instance is not the case for our running example. Therefore, we consider a more sophisticated definition of $\vdash_{\text {ok }}\left(\mathrm{G}, \mathcal{M}, \mathcal{M}^{\prime}\right)$ which goes beyond regular derivations. The judgement is defined in Figure 14.

The first condition we require is the equivalence $\mathcal{M}^{\prime} \equiv \mathcal{M} \cdot \mathcal{M}^{\prime \prime}$. This is needed because, if a message in $\mathcal{M}$ is not in $\mathcal{M}^{\prime}$, then a coinductive derivation of the judgement $\vdash_{\mathrm{b}} \mathrm{G} \| \mathcal{M}^{\prime}$ would get stuck on Rule [B-IN], i.e., the judgement would not be derivable, making the algorithm unsound. However, we allow the presence of additional messages, so that the queue between two occurrences of the same global type can grow.

In order to ensure that the messages in the queue $\mathcal{M}^{\prime \prime}$ do not interfere with the balancing of $\mathrm{G}$, we demand that they can be exchanged with the outputs of $\mathrm{G}$. This condition is checked

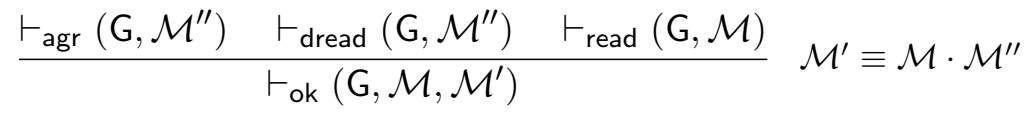

$$
\begin{aligned}
& \text { [A-END] } \overline{\mathcal{H} \vdash_{\text {agr }}(\text { End, } \mathcal{M})} \quad[\mathrm{A}-\mathrm{CYCLE}] \overline{\mathcal{H},(\mathrm{G}, \mathcal{M}) \vdash_{\text {agr }}(\mathrm{G}, \mathcal{M})} \\
& \text { [A-OUT] } \frac{\mathcal{H},\left(\mathrm{p} \mathrm{q} !\left\{\lambda_{i} ; \mathrm{G}_{i}\right\}_{i \in I}, \mathcal{M}\right) \vdash_{\mathrm{agr}}\left(\mathrm{G}_{i}, \mathcal{M}_{i}\right) \quad \forall i \in I}{\mathcal{H} \vdash_{\mathrm{agr}}\left(\mathrm{p} \mathrm{q} !\left\{\lambda_{i} ; \mathrm{G}_{i}\right\}_{i \in I}, \mathcal{M}\right)} \quad \mathcal{M} \cdot\left\langle\mathrm{p}, \lambda_{i}, \mathrm{q}\right\rangle \equiv_{\mathrm{G}_{i}}\left\langle\mathrm{p}, \lambda_{i}, \mathrm{q}\right\rangle \cdot \mathcal{M}_{i} \forall i \in I \\
& {[\mathrm{~A}-\mathrm{IN}] \frac{\mathcal{H},\left(\mathrm{pq} ?\left\{\lambda_{i} ; \mathrm{G}_{i}\right\}_{i \in I}, \mathcal{M}\right) \vdash_{\mathrm{agr}}\left(\mathrm{G}_{i}, \mathcal{M}\right) \forall i \in I}{\mathcal{H} \vdash_{\mathrm{agr}}\left(\mathrm{pq} ?\left\{\lambda_{i} ; \mathrm{G}_{i}\right\}_{i \in I}, \mathcal{M}\right)}}
\end{aligned}
$$

$$
\begin{aligned}
& {[\text { END-DR }] \frac{\mathcal{G}_{\text {dread }}(\text { End, } \emptyset)}{[\text { CyCLE-DR }]} \frac{\vdash_{\text {read }}(\mathrm{G}, \mathcal{M})}{\mathcal{G}, \vdash_{\text {dread }}(\mathrm{G}, \mathcal{M})}} \\
& \text { [Out-DR] } \frac{\mathcal{G}, \mathrm{pq} !\left\{\lambda_{i} ; \mathrm{G}_{i}\right\}_{i \in I} \vdash_{\text {dread }}\left(\mathrm{G}_{i}, \mathcal{M}\right) \forall i \in I}{\mathcal{G} \vdash_{\text {dread }}\left(\mathrm{p} \mathrm{q} !\left\{\lambda_{i} ; \mathrm{G}_{i}\right\}_{i \in I}, \mathcal{M}\right)} \\
& {[\mathrm{IN}-\mathrm{DR}] \frac{\mathcal{G}, \mathrm{pq} ?\left\{\lambda_{i} ; \mathrm{G}_{i}\right\}_{i \in I} \vdash_{\text {dread }}\left(\mathrm{G}_{i}, \mathcal{M}\right) \forall i \in I}{\mathcal{G} \vdash_{\text {dread }}\left(\mathrm{pq} ?\left\{\lambda_{i} ; \mathrm{G}_{i}\right\}_{i \in I}, \mathcal{M}\right)}}
\end{aligned}
$$

Figure 14: Ok, agreement and deep read judgments. 
by the judgement $\vdash_{\text {agr }}\left(\mathrm{G}, \mathcal{M}^{\prime \prime}\right)$, whose definition relies on an extension of the equivalence $\equiv$ on queues, parametrised over a global type $\mathrm{G}$, denoted by $\equiv_{\mathrm{G}}$, see Definition 6.1. The key rule is $[\mathrm{A}-\mathrm{OUT}]$. This rule requires that for each branch $\mathrm{G}_{i}$ of an output the message put at the end of the queue can go on top when queues are considered modulo $\equiv_{\mathrm{G}_{i}}$.

Finally, we have to check that all messages in $\mathcal{M}^{\prime}$ will be eventually read. To this end, we use judgements $\vdash_{\text {read }}(\mathrm{G}, \mathcal{M})$ and $\vdash_{\text {dread }}\left(\mathrm{G}, \mathcal{M}^{\prime \prime}\right)$, where the latter ensures that messages in $\mathcal{M}^{\prime \prime}$ can be read in any path of $G$ an unbounded number of times. This is needed because many copies of messages in $\mathcal{M}^{\prime \prime}$ will be accumulated at the end of the queue and we do not know in which path of $G$ they will be read, thus they must be readable in all of them. In $\vdash_{\text {dread }}$ all branches of a global type are explored without removing any message until getting End or an already considered global type. The first case is successful if the queue is empty, in the second case the judgement $\vdash_{\text {read }}$ is required. For instance, if $G=p q ?\left\{\lambda\right.$; End, $\lambda^{\prime}$; End $\}$, then $\nvdash$ dread $(\mathrm{G},\langle\mathrm{p}, \lambda, \mathrm{q}\rangle)$, since the message $\langle\mathrm{p}, \lambda, \mathrm{q}\rangle$ is not read in all branches. However, $\vdash_{\text {read }}(\mathrm{G},\langle\mathrm{p}, \lambda, \mathrm{q}\rangle)$, since the message $\langle\mathrm{p}, \lambda, \mathrm{q}\rangle$ is read in one of the branches.

To formally give the equivalence $\equiv_{\mathrm{G}}$ on queues we first define when two messages are G-indistinguishable, meaning that the behaviour of $\mathrm{G}$ is independent on reading one or the other. Then, the equivalence $\equiv_{\mathrm{G}}$ is defined by extending the equivalence on queues to allow the replacement of messages with G-indistinguishable ones.

Definition 6.1. (1) The messages $\langle p, \lambda, q\rangle$ and $\left\langle p, \lambda^{\prime}, q\right\rangle$ are G-indistinguishable if $\lambda$ and $\lambda^{\prime}$ occur in $\mathrm{G}$ and for every input choice $\mathrm{pq} ?\left\{\lambda_{i} ; \mathrm{G}_{i}\right\}_{i \in I}$ occurring in $\mathrm{G}$,

- either $\left\{\lambda, \lambda^{\prime}\right\} \cap\left\{\lambda_{i} \mid i \in I\right\}=\emptyset$

- or $\lambda=\lambda_{h}, \lambda^{\prime}=\lambda_{k}$ and $\mathrm{G}_{h}=\mathrm{G}_{k}$ for some $h, k \in I$.

(2) The equivalence $\equiv_{G}$ between queues is obtained extending $\equiv$ by the following clause:

$$
\mathcal{M}_{1} \cdot\langle\mathrm{p}, \lambda, \mathrm{q}\rangle \cdot \mathcal{M}_{2} \equiv_{\mathrm{G}} \mathcal{M}_{1} \cdot\left\langle\mathrm{p}, \lambda^{\prime}, \mathrm{q}\right\rangle \cdot \mathcal{M}_{2} \quad \text { if } \quad\langle\mathrm{p}, \lambda, \mathrm{q}\rangle,\left\langle\mathrm{p}, \lambda^{\prime}, \mathrm{q}\right\rangle \text { are G-indistinguishable }
$$

Notice that, if $\mathcal{M} \equiv_{\mathrm{G}} \mathcal{M}_{1} \cdot\langle\mathrm{p}, \lambda, \mathrm{q}\rangle \cdot \mathcal{M}_{2}$, then $\mathcal{M} \equiv \mathcal{M}_{1}^{\prime} \cdot\left\langle\mathrm{p}, \lambda^{\prime}, \mathrm{q}\right\rangle \cdot \mathcal{M}_{2}^{\prime}$ with $\langle\mathrm{p}, \lambda, \mathrm{q}\rangle,\left\langle\mathrm{p}, \lambda^{\prime}, \mathrm{q}\right\rangle$ G-indistinguishable and $\mathcal{M}_{1} \equiv_{\mathrm{G}} \mathcal{M}_{1}^{\prime}$ and $\mathcal{M}_{2} \equiv_{\mathrm{G}} \mathcal{M}_{2}^{\prime}$.

Taking $\mathbf{G}=\mathrm{pq} ?\left\{\lambda\right.$; End, $\lambda^{\prime}$; End $\}$, we get that $\langle\mathrm{p}, \lambda, \mathrm{q}\rangle \cdot\left\langle\mathrm{p}, \lambda^{\prime}, \mathrm{q}\right\rangle \equiv_{\mathrm{G}}\left\langle\mathrm{p}, \lambda^{\prime}, \mathrm{q}\right\rangle \cdot\langle\mathrm{p}, \lambda, \mathrm{q}\rangle$, whereas $\langle\mathrm{p}, \lambda, \mathrm{q}\rangle \cdot\left\langle\mathrm{p}, \lambda^{\prime}, \mathrm{q}\right\rangle \not \equiv\left\langle\mathrm{p}, \lambda^{\prime}, \mathrm{q}\right\rangle \cdot\langle\mathrm{p}, \lambda, \mathrm{q}\rangle$. We will see that this parametrised equivalence plays a crucial role in deriving the agreement judgement for the example of the hospital.

To prove the soundness theorem (Theorem 6.8), we need properties of the auxiliary judgements.

First properties concern the two readability judgements. Lemma 6.2 shows that queues with the readability property can be split preserving readability. Lemma 6.3(1) and Lemma 6.3(2) prove weakening and cut elimination for deep readability, respectively. Relying on these two properties, Lemma 6.3(3) proves a strong form of inversion for deep readability, deriving it for any subtree of a deeply readable global type with the same queue (when the set of hypotheses is empty). Lemma 6.3(4) and Lemma 6.3(5) connect readability with deep readability. Lemma 6.3(6) extends Lemma 6.2 to deep readability. Finally, Proposition 6.4 shows that readability and deep readability are preserved by the parametrised equivalence $\equiv_{\mathrm{G}}$.

Lemma 6.2. If $\vdash_{\text {read }}\left(\mathrm{G}, \mathcal{M} \cdot \mathcal{M}^{\prime}\right)$, then $\vdash_{\text {read }}(\mathrm{G}, \mathcal{M})$ and $\vdash_{\text {read }}\left(\mathrm{G}, \mathcal{M}^{\prime}\right)$.

Proof. We show that $\vdash_{\text {read }}(\mathrm{G}, \mathcal{M})$ implies $\vdash_{\text {read }}\left(\mathrm{G}, \mathcal{M}^{\prime}\right)$ when $\mathcal{M}^{\prime}$ is a subsequence of $\mathcal{M}$ (modulo $\equiv$ ). The proof is by induction on the derivation of $\vdash_{\text {read }}(G, \mathcal{M})$. The only interesting cases are when the last applied rule is either [IN-R1] or [IN-R2]. 
[IN-R1]: We have $\mathrm{G}=\mathrm{pq} ?\left\{\lambda_{i} ; \mathrm{G}_{i}\right\}_{i \in I}$ and $\mathcal{M} \equiv\left\langle\mathrm{p}, \lambda_{h}, \mathrm{q}\right\rangle \cdot \widehat{\mathcal{M}}$ with $h \in I$ and $\vdash_{\text {read }}\left(\mathrm{G}_{i}, \widehat{\mathcal{M}}\right)$ for all $i \in I$. If $\mathcal{M}^{\prime} \equiv\left\langle\mathrm{p}, \lambda_{k}, \mathrm{q}\right\rangle \cdot \mathcal{M}^{\prime \prime}$ for some $k \in I$ (note that $k$ and $h$ may differ), then $\mathcal{M}^{\prime \prime}$ is a subsequence of $\widehat{\mathcal{M}}$. Then, by induction hypothesis, we get $\vdash_{\text {read }}\left(\mathrm{G}_{i}, \mathcal{M}^{\prime \prime}\right)$ for all $i \in I$ and so we conclude by applying Rule [IN-R1]. Otherwise, $\mathcal{M}^{\prime}$ is a subsequence of $\widehat{\mathcal{M}}$, then by induction hypothesis we get $\vdash_{\text {read }}\left(\mathrm{G}_{i}, \mathcal{M}^{\prime}\right)$ for all $i \in I$ and so we conclude by applying Rule [IN-R2].

[IN-R2]: We have $\mathrm{G}=\mathrm{pq}$ ? $\left\{\lambda_{i} ; \mathrm{G}_{i}\right\}_{i \in I}$ and $\mathcal{M} \not \equiv\left\langle\mathrm{p}, \lambda_{i}, \mathrm{q}\right\rangle \cdot \widehat{\mathcal{M}}$ and $\vdash_{\text {read }}\left(\mathrm{G}_{i}, \mathcal{M}\right)$ for all $i \in I$. If $\mathcal{M}^{\prime} \equiv\left\langle\mathrm{p}, \lambda_{h}, \mathrm{q}\right\rangle \cdot \mathcal{M}^{\prime \prime}$ with $h \in I$, since $\mathcal{M}^{\prime \prime}$ is still a subsequence of $\mathcal{M}$, by induction hypothesis, we get $\vdash_{\text {read }}\left(\mathrm{G}_{i}, \mathcal{M}^{\prime \prime}\right)$ for all $i \in I$ and so we conclude by applying Rule [IN-R1]. Otherwise, again by induction hypothesis we get $\vdash_{\text {read }}\left(\mathrm{G}_{i}, \mathcal{M}^{\prime}\right)$, for all $i \in I$, and so we conclude by applying Rule [IN-R2].

Lemma 6.3. The following properties hold.

(1) If $\mathcal{G} \vdash_{\text {dread }}(\mathrm{G}, \mathcal{M})$, then $\mathcal{G}, \mathcal{G}^{\prime} \vdash_{\text {dread }}(\mathrm{G}, \mathcal{M})$.

(2) If $\mathcal{G} \vdash_{\text {dread }}(\mathrm{G}, \mathcal{M})$ and $\mathcal{G}, \mathrm{G} \vdash$ dread $\left(\mathrm{G}^{\prime}, \mathcal{M}\right)$, then $\mathcal{G} \vdash_{\text {dread }}\left(\mathrm{G}^{\prime}, \mathcal{M}\right)$.

(3) If $\vdash_{\text {dread }}(\mathrm{G}, \mathcal{M})$, then $\vdash_{\text {dread }}\left(\mathrm{G}^{\prime}, \mathcal{M}\right)$ for any type $\mathrm{G}^{\prime}$ occurring in $\mathrm{G}$.

(4) If $\mathcal{G} \vdash_{\text {dread }}(\mathrm{G}, \mathcal{M})$, then $\vdash_{\text {read }}(\mathrm{G}, \mathcal{M})$.

(5) If $\vdash_{\text {read }}\left(\mathrm{G}, \mathcal{M}_{1}\right)$ and $\vdash_{\text {dread }}\left(\mathrm{G}, \mathcal{M}_{2}\right)$, then $\vdash_{\text {read }}\left(\mathrm{G}, \mathcal{M}_{1} \cdot \mathcal{M}_{2}\right)$.

(6) If $\mathcal{G} \vdash_{\text {dread }}\left(\mathrm{G}, \mathcal{M}_{1} \cdot \mathcal{M}_{2}\right)$, then $\mathcal{G} \vdash_{\text {dread }}\left(\mathrm{G}, \mathcal{M}_{1}\right)$ and $\mathcal{G} \vdash_{\text {dread }}\left(\mathrm{G}, \mathcal{M}_{2}\right)$.

Proof. (1). The proof is by a straightforward induction on the derivation of $\mathcal{G} \vdash_{\text {dread }}(\mathrm{G}, \mathcal{M})$. (2). The proof is by a straightforward induction on the derivation of $\mathcal{G}, G \vdash_{\text {dread }}\left(\mathrm{G}^{\prime}, \mathcal{M}\right)$ using Item $(1)$ on $\mathcal{G} \vdash_{\text {dread }}(G, \mathcal{M})$ to properly extend $\mathcal{G}$ in order to apply the induction hypothesis.

(3). The proof is by induction on the distance of the subtree $G^{\prime}$ from the root in the tree of $G$. If the distance is 0 , then $G^{\prime}=G$ and so the statement is trivial. Otherwise, $G^{\prime}$ is the direct subtree of another subtree $G^{\prime \prime}$ of $G$, which is closer to the root; then by induction hypothesis, we have $\vdash_{\text {dread }}\left(\mathrm{G}^{\prime \prime}, \mathcal{M}\right)$. Let $\mathrm{G}^{\prime \prime}=\mathrm{pq} \dagger\left\{\lambda_{i} ; \mathrm{G}_{i}\right\}_{i \in I}$ and $\mathrm{G}^{\prime}=\mathrm{G}_{h}$ for some $h \in I$. We get $G^{\prime \prime} \vdash_{\text {dread }}\left(G^{\prime}, \mathcal{M}\right)$, as it is a premise of the rule used to derive $\vdash_{\text {dread }}\left(G^{\prime \prime}, \mathcal{M}\right)$, then, by Item $(2)$, we get $\vdash_{\text {dread }}\left(\mathrm{G}^{\prime}, \mathcal{M}\right)$, as needed.

(4). The proof is by induction on the derivation of $\mathcal{G} \vdash_{\text {dread }}(G, \mathcal{M})$, splitting cases on the last applied rule in the derivation. The only non-trivial case is for Rule [IN-DR]. We have $\mathrm{G}=\mathrm{pq}$ ? $\left\{\lambda_{i} ; \mathrm{G}_{i}\right\}_{i \in I}$ and, by induction hypothesis, we get $\vdash_{\text {read }}\left(\mathrm{G}_{i}, \mathcal{M}\right)$ for all $i \in I$. We have two cases: if $\mathcal{M} \not \equiv\left\langle\mathrm{p}, \lambda_{i}, \mathrm{q}\right\rangle \cdot \mathcal{M}^{\prime}$ for all $i \in I$, the statement follows by Rule [IN-R2]. Otherwise, $\mathcal{M}=\left\langle\mathrm{p}, \lambda_{h}, \mathrm{q}\right\rangle \cdot \mathcal{M}^{\prime}$ for some $h \in I$ and, by Lemma 6.2 applied to $\vdash_{\text {read }}\left(\mathrm{G}_{i}, \mathcal{M}\right)$, we get $\vdash_{\text {read }}\left(\mathrm{G}_{i}, \mathcal{M}^{\prime}\right)$ for all $i \in I$, thus the statement follows by Rule [IN-R1].

(5). The proof is by induction on the derivation of $\vdash_{\text {read }}\left(\mathrm{G}, \mathcal{M}_{1}\right)$. We split cases on the last applied rule.

[EMPTY-R]: We have $\mathcal{M}_{1}=\emptyset$ and so the statement follows by Item (4).

[OUT-R]: We have $\mathrm{G}=\mathrm{pq} !\left\{\lambda_{i} ; \mathrm{G}_{i}\right\}_{i \in I}$ and, since by hypothesis we have $\vdash_{\text {dread }}\left(\mathrm{G}, \mathcal{M}_{2}\right)$, by Item (3) we get $\vdash_{\text {dread }}\left(\mathrm{G}_{i}, \mathcal{M}_{2}\right)$, for all $i \in I$. Then, by induction hypothesis, we get $\vdash_{\text {read }}\left(\mathrm{G}_{i}, \mathcal{M}_{1} \cdot \mathcal{M}_{2}\right)$, for all $i \in I$ and so the statement follows by Rule [OUT-R].

[IN-R1]: We have $\mathrm{G}=\mathrm{pq}$ ? $\left\{\lambda_{i} ; \mathrm{G}_{i}\right\}_{i \in I}$ and $\mathcal{M}_{1} \equiv\left\langle\mathrm{p}, \lambda_{h}, \mathrm{q}\right\rangle \cdot \mathcal{M}^{\prime}$ for some $h \in I$, and, since by hypothesis we have $\vdash_{\text {dread }}\left(\mathrm{G}, \mathcal{M}_{2}\right)$, by Item (3) we get $\vdash_{\text {dread }}\left(\mathrm{G}_{i}, \mathcal{M}_{2}\right)$ for all $i \in I$. Then, by induction hypothesis, we get $\vdash_{\text {read }}\left(G_{i}, \mathcal{M}^{\prime} \cdot \mathcal{M}_{2}\right)$ for all $i \in I$ and so the statement follows by Rule [IN-R1]. 


$$
\begin{aligned}
& {\left[\mathrm{A}-\mathrm{End}^{\prime}\right] \overline{\overline{(\text { End, } \mathcal{M})}}} \\
& {\left[\text { A-OuT }^{\prime}\right] \frac{\left(\mathrm{G}_{i}, \mathcal{M}_{i}\right) \quad \forall i \in I}{\left(\mathrm{pq} !\left\{\lambda_{i} ; \mathrm{G}_{i}\right\}_{i \in I}, \mathcal{M}\right)} \mathcal{M} \cdot\left\langle\mathrm{p}, \lambda_{i}, \mathrm{q}\right\rangle \equiv_{\mathrm{G}_{i}}\left\langle\mathrm{p}, \lambda_{i}, \mathrm{q}\right\rangle \cdot \mathcal{M}_{i} \forall i \in I} \\
& {\left[\mathrm{~A}-\mathrm{IN}^{\prime}\right] \frac{\left(\mathrm{G}_{i}, \mathcal{M}\right) \quad \forall i \in I}{\left(\mathrm{pq} ?\left\{\lambda_{i} ; \mathrm{G}_{i}\right\}_{i \in I}, \mathcal{M}\right)}}
\end{aligned}
$$

Figure 15: Agreement judgement (coinductive version).

[IN-R2]: We have $\mathrm{G}=\mathrm{pq} ?\left\{\lambda_{i} ; \mathrm{G}_{i}\right\}_{i \in I}$ and $\mathcal{M}_{1} \not \equiv\left\langle\mathrm{p}, \lambda_{i}, \mathrm{q}\right\rangle \cdot \mathcal{M}^{\prime}$ for all $i \in I$, and, since by hypothesis we have $\vdash_{\text {dread }}\left(G, \mathcal{M}_{2}\right)$, by Item $(3)$, we get $\vdash_{\text {dread }}\left(G_{i}, \mathcal{M}_{2}\right)$ for all $i \in I$. Then, by induction hypothesis, we get $\vdash_{\text {read }}\left(\mathrm{G}_{i}, \mathcal{M}_{1} \cdot \mathcal{M}_{2}\right)$ for all $i \in I$. Now, if $\mathcal{M}_{1} \cdot \mathcal{M}_{2} \equiv\left\langle\mathrm{p}, \lambda_{h}, \mathrm{q}\right\rangle \cdot \widehat{\mathcal{M}}$ for some $h \in I$, Lemma 6.2 implies $\vdash_{\text {read }}\left(\mathrm{G}_{i}, \widehat{\mathcal{M}}\right)$ for all $i \in I$ and so the statement follows by Rule [IN-R1]. Otherwise the statement follows by Rule [IN-R2].

(6). The proof is by a straightforward induction on the derivation of $\mathcal{G} \vdash_{\text {dread }}\left(\mathrm{G}, \mathcal{M}_{1} \cdot \mathcal{M}_{2}\right)$ using Lemma 6.2.

Proposition 6.4. Let $\mathcal{M} \equiv_{\mathrm{G}} \mathcal{M}^{\prime}$.

(1) If $\vdash_{\text {read }}(\mathrm{G}, \mathcal{M})$, then $\vdash_{\text {read }}\left(\mathrm{G}, \mathcal{M}^{\prime}\right)$.

(2) If $\mathcal{G} \vdash_{\text {dread }}(\mathrm{G}, \mathcal{M})$, then $\mathcal{G} \vdash_{\text {dread }}\left(\mathrm{G}, \mathcal{M}^{\prime}\right)$.

Proof. (1). The proof is by induction on the derivation of $\vdash_{\text {read }}(G, \mathcal{M})$, splitting cases on the last applied rule. The only relevant case is for Rule [IN-R1]. We have $\mathrm{G}=\mathrm{pq}$ ? $\left\{\lambda_{i} ; \mathrm{G}_{i}\right\}_{i \in I}$ and $\mathcal{M} \equiv\left\langle\mathrm{p}, \lambda_{h}, \mathrm{q}\right\rangle \cdot \widehat{\mathcal{M}}$ with $h \in I$. Then, we have $\mathcal{M}^{\prime} \equiv\left\langle\mathrm{p}, \lambda^{\prime}, \mathrm{q}\right\rangle \cdot \widehat{\mathcal{M}}^{\prime}$ with $\left\langle\mathrm{p}, \lambda_{h}, \mathrm{q}\right\rangle,\left\langle\mathrm{p}, \lambda^{\prime}, \mathrm{q}\right\rangle \mathrm{G}-$ indistinguishable and $\widehat{\mathcal{M}} \equiv_{\mathrm{G}} \widehat{\mathcal{M}}^{\prime}$, which implies $\widehat{\mathcal{M}} \equiv_{\mathrm{G}_{i}} \widehat{\mathcal{M}}^{\prime}$ for all $i \in I$. Hence, by induction hypothesis, we get $\vdash_{\text {read }}\left(\mathrm{G}_{i}, \widehat{\mathcal{M}}^{\prime}\right)$ for all $i \in I$. By definition of G-indistinguishability we have $\lambda^{\prime}=\lambda_{k}$ for some $k \in I$. Finally, we get the statement by Rule [IN-R1].

(2). It follows by a straightforward induction on the derivation of $\mathcal{G} \vdash_{\text {dread }}(\mathrm{G}, \mathcal{M})$, using Item (1).

To reason about the agreement judgement with an empty set of hypotheses, it is often convenient to consider an equivalent coinductive formulation reported in Figure 15. These two formulations are equivalent because all infinite derivations using rules in Figure 15 are actually regular. In fact, in a derivation of $(G, \mathcal{M})$, there are only finitely many different global types, as $\mathrm{G}$ is regular. Moreover, there are finitely many different queues, since they must all have the same length as the initial one and labels must occur in G, by definition of G-equivalence. Hence, such a derivation has only finitely many different nodes, namely, it is regular. This implies that the judgement in Figure 15 is equivalent to the agreement judgement in Figure 14 by [Dag21, Theorem 5.2].

The next lemma proves inversion for Rules [A-OUT] and [A-IN].

Lemma 6.5. Let $\vdash_{\mathrm{agr}}(\mathrm{G}, \mathcal{M})$, then the following hold.

(1) If $\mathrm{G}=\mathrm{pq} !\left\{\lambda_{i} ; \mathrm{G}_{i}\right\}_{i \in I}$, then, for each $i \in I$, there is $\mathcal{M}_{i}$ such that $\vdash_{\mathrm{agr}}\left(\mathrm{G}_{i}, \mathcal{M}_{i}\right)$ and $\mathcal{M} \cdot\left\langle\mathrm{p}, \lambda_{i}, \mathrm{q}\right\rangle \equiv_{\mathrm{G}_{i}}\left\langle\mathrm{p}, \lambda_{i}, \mathrm{q}\right\rangle \cdot \mathcal{M}_{i}$.

(2) If $\mathrm{G}=\mathrm{pq}$ ? $\left\{\lambda_{i} ; \mathrm{G}_{i}\right\}_{i \in I}$, then $\vdash_{\mathrm{agr}}\left(\mathrm{G}_{i}, \mathcal{M}\right)$ for all $i \in I$. 
Proof. This is straightforward by the equivalent coinductive characterisation of $\vdash_{\text {agr }}(\mathrm{G}, \mathcal{M})$ given in Figure 15.

We now prove that agreement and deep readability judgements (when the set of hypothesis is empty) are preserved by concatenation of queues.

Lemma 6.6. The following properties hold.

(1) If $\vdash_{\text {agr }}\left(\mathrm{G}, \mathcal{M}_{1}\right)$ and $\vdash_{\text {agr }}\left(\mathrm{G}, \mathcal{M}_{2}\right)$, then $\vdash_{\text {agr }}\left(\mathrm{G}, \mathcal{M}_{1} \cdot \mathcal{M}_{2}\right)$.

(2) If $\vdash_{\text {dread }}\left(\mathrm{G}, \mathcal{M}_{1}\right)$ and $\vdash_{\text {dread }}\left(\mathrm{G}, \mathcal{M}_{2}\right)$, then $\vdash_{\text {dread }}\left(\mathrm{G}, \mathcal{M}_{1} \cdot \mathcal{M}_{2}\right)$.

Proof. (1). The proof is by coinduction relying on the coinductive characterisation of $\vdash_{\text {agr }}(\mathrm{G}, \mathcal{M})$ in Figure 15 . Consider the set $\mathcal{A}$ defined as follows:

$(\mathrm{G}, \widehat{\mathcal{M}})$ belongs to $\mathcal{A}$ if $\widehat{\mathcal{M}} \equiv \mathcal{M}_{1} \cdot \mathcal{M}_{2}$ and $\vdash_{\text {agr }}\left(\mathrm{G}, \mathcal{M}_{1}\right)$ and $\vdash_{\text {agr }}\left(\mathrm{G}, \mathcal{M}_{2}\right)$ hold

We have to prove that $\mathcal{A}$ is consistent, that is, every element $(\mathrm{G}, \widehat{\mathcal{M}})$ in $\mathcal{A}$ is the conclusion of a rule in Figure 15, whose premises are still in $\mathcal{A}$. We split cases on $\mathrm{G}$.

- If $\mathrm{G}=\mathrm{End}$, the statement follows immediately by Rule [A-END'], as it has no premises.

- If $\mathrm{G}=\mathrm{p} \mathrm{q} !\left\{\lambda_{i} ; \mathrm{G}_{i}\right\}_{i \in I}$, then by Lemma 6.5(1) we get $\vdash_{\mathrm{agr}}\left(\mathrm{G}_{i}, \mathcal{M}_{i}^{k}\right)$ and $\mathcal{M}_{k} \cdot\left\langle\mathrm{p}, \lambda_{i}, \mathrm{q}\right\rangle \equiv_{\mathrm{G}_{i}}$ $\left\langle\mathrm{p}, \lambda_{i}, \mathrm{q}\right\rangle \cdot \mathcal{M}_{i}^{k}$ for $k=1,2$ and for all $i \in I$. Therefore, $\left(\mathrm{G}_{i}, \mathcal{M}_{i}^{1} \cdot \mathcal{M}_{i}^{2}\right)$ belongs to $\mathcal{A}$ for all $i \in I$. Notice that

$$
\mathcal{M}_{1} \cdot \mathcal{M}_{2} \cdot\left\langle\mathrm{p}, \lambda_{i}, \mathrm{q}\right\rangle \equiv_{\mathrm{G}_{i}} \mathcal{M}_{1} \cdot\left\langle\mathrm{p}, \lambda_{i}, \mathrm{q}\right\rangle \cdot \mathcal{M}_{i}^{2} \equiv_{\mathrm{G}_{i}}\left\langle\mathrm{p}, \lambda_{i}, \mathrm{q}\right\rangle \cdot \mathcal{M}_{i}^{1} \cdot \mathcal{M}_{i}^{2}
$$

for all $i \in I$, hence, we conclude by Rule [A-OUT'].

- If $\mathrm{G}=\mathrm{pq}$ ? $\left\{\lambda_{i} ; \mathrm{G}_{i}\right\}_{i \in I}$, then by Lemma $6.5(2)$ we get $\vdash_{\text {agr }}\left(\mathrm{G}_{i}, \mathcal{M}_{k}\right)$ for $k=1,2$ and for all $i \in I$. Therefore, $\left(\mathrm{G}_{i}, \mathcal{M}_{1} \cdot \mathcal{M}_{2}\right)$ belongs to $\mathcal{A}$ for all $i \in I$, hence we conclude by Rule [A-IN'].

(2). We generalise the statement proving that, if $\mathcal{G} \vdash_{\text {dread }}\left(\mathrm{G}, \mathcal{M}_{1}\right)$ and $\vdash_{\text {dread }}\left(\mathrm{G}, \mathcal{M}_{2}\right)$, then $\mathcal{G} \vdash_{\text {dread }}\left(\mathrm{G}, \mathcal{M}_{1} \cdot \mathcal{M}_{2}\right)$. We get the thesis when $\mathcal{G}$ is empty. The proof is by induction on the derivation of $\mathcal{G} \vdash_{\text {dread }}\left(\mathrm{G}, \mathcal{M}_{1}\right)$. For Rule [END-DR], we have $\mathrm{G}=$ End, hence both $\mathcal{M}_{1}$ and $\mathcal{M}_{2}$ are empty. Then the thesis follows immediately by Rule [END-DR]. For Rules [OUT$\mathrm{DR}$ ] and [IN-DR], we have $\mathrm{G}=\mathrm{pq} \dagger\left\{\lambda_{i} ; \mathrm{G}_{i}\right\}_{i \in I}$ and $\mathcal{G}, \mathrm{G} \vdash_{\text {dread }}\left(\mathrm{G}_{i}, \mathcal{M}_{1}\right)$ for all $i \in I$. By Lemma 6.3(3), we get $\vdash_{\text {dread }}\left(\mathrm{G}_{i}, \mathcal{M}_{2}\right)$, for all $i \in I$, hence the thesis follows from the induction hypothesis applying again Rules [OUT-DR] and [IN-DR], respectively. For Rule [CYCLE-DR], we have $\mathcal{G}=\mathcal{G}^{\prime}, \mathrm{G}$ and $\vdash_{\text {read }}\left(\mathrm{G}, \mathcal{M}_{1}\right)$, then Lemma 6.3(5) implies $\vdash_{\text {read }}\left(\mathrm{G}, \mathcal{M}_{1} \cdot \mathcal{M}_{2}\right)$ using $\vdash_{\text {dread }}\left(\mathrm{G}, \mathcal{M}_{2}\right)$. So the thesis follows applying Rule [CYCLE-DR].

Lastly, we prove that deep readability is preserved when the queue is changed as in Rule [A-OUT] of Figure 14.

Lemma 6.7. If $\vdash_{\text {dread }}(\mathrm{G}, \mathcal{M})$ and $\mathcal{M} \cdot\langle\mathrm{p}, \lambda, \mathrm{q}\rangle \equiv_{\mathrm{G}}\langle\mathrm{p}, \lambda, \mathrm{q}\rangle \cdot \mathcal{M}^{\prime}$, then $\vdash_{\text {dread }}\left(\mathrm{G}, \mathcal{M}^{\prime}\right)$.

Proof. From the hypothesis we have $\mathcal{M} \cdot\langle\mathrm{p}, \lambda, \mathrm{q}\rangle \equiv\left\langle\mathrm{p}, \lambda^{\prime}, \mathrm{q}\right\rangle \cdot \widehat{\mathcal{M}}$ with $\langle\mathrm{p}, \lambda, \mathrm{q}\rangle$ and $\left\langle\mathrm{p}, \lambda^{\prime}, \mathrm{q}\right\rangle$ G-indistinguishable and $\widehat{\mathcal{M}} \equiv_{\mathrm{G}} \mathcal{M}^{\prime}$. We have two cases.

- If there is no message from $\mathrm{p}$ to $\mathrm{q}$ in $\mathcal{M}$, then $\mathcal{M} \cdot\langle\mathrm{p}, \lambda, \mathrm{q}\rangle \equiv\langle\mathrm{p}, \lambda, \mathrm{q}\rangle \cdot \mathcal{M}$, hence we have $\lambda=\lambda^{\prime}$ and $\mathcal{M} \equiv \widehat{\mathcal{M}}$. Therefore, $\mathcal{M} \equiv_{\mathrm{G}} \mathcal{M}^{\prime}$ and the statement follows by Proposition $6.4(2)$.

- Otherwise, $\mathcal{M} \equiv\left\langle\mathrm{p}, \lambda^{\prime}, \mathrm{q}\right\rangle \cdot \mathcal{M}_{1}$ and so $\mathcal{M}_{1} \cdot\langle\mathrm{p}, \lambda, \mathrm{q}\rangle \equiv \widehat{\mathcal{M}} \equiv_{\mathrm{G}} \mathcal{M}^{\prime}$, which implies $\mathcal{M}^{\prime} \equiv \mathcal{M}_{2} \cdot\left\langle\mathrm{p}, \lambda^{\prime \prime}, \mathrm{q}\right\rangle$ with $\langle\mathrm{p}, \lambda, \mathrm{q}\rangle$ and $\left\langle\mathrm{p}, \lambda^{\prime \prime}, \mathrm{q}\right\rangle$ G-indistinguishable and $\mathcal{M}_{1} \equiv_{\mathrm{G}} \mathcal{M}_{2}$. By Lemma $6.3(6)$, we have $\vdash_{\text {dread }}\left(\mathrm{G},\left\langle\mathrm{p}, \lambda^{\prime}, \mathrm{q}\right\rangle\right)$ and $\vdash_{\text {dread }}\left(\mathrm{G}, \mathcal{M}_{1}\right)$ and, by Proposition $6.4(2)$, we get $\vdash_{\text {dread }}\left(\mathrm{G}, \mathcal{M}_{2}\right)$. Note that $\left\langle\mathrm{p}, \lambda^{\prime}, \mathrm{q}\right\rangle \equiv_{\mathrm{G}}\langle\mathrm{p}, \lambda, \mathrm{q}\rangle \equiv_{\mathrm{G}}\left\langle\mathrm{p}, \lambda^{\prime \prime}, \mathrm{q}\right\rangle$, hence 
by Proposition $6.4(2)$ we get $\vdash_{\text {dread }}\left(\mathrm{G},\left\langle\mathrm{p}, \lambda^{\prime \prime}, \mathrm{q}\right\rangle\right)$. Finally, by Lemma $6.6(2)$, we get $\vdash_{\text {dread }}\left(\mathrm{G}, \mathcal{M}_{2} \cdot\left\langle\mathrm{p}, \lambda^{\prime \prime}, \mathrm{q}\right\rangle\right)$, which is the statement.

Now we are able to state and prove the soundness result for the inductive balancing judgement.

Theorem 6.8 (Soundness). If $\vdash_{\mathrm{b}}^{\mathcal{I}} \mathrm{G} \| \mathcal{M}$, then $\vdash_{\mathrm{b}} \mathrm{G} \| \mathcal{M}$.

Proof. First of all we observe that the definition of the judgement $\mathcal{H} \vdash_{\mathrm{b}}^{\mathcal{I}} \mathrm{G} \| \mathcal{M}$ can be equivalently expressed assuming $\mathcal{H}$ to be a sequence rather than a set, the only difference is Rule [IB-CYCLE] which will have the following shape

$$
[\text { IB-CYCLE }] \stackrel{\vdash_{\text {read }}(\mathrm{G}, \mathcal{M}) \quad \vdash_{\text {agr }}\left(\mathrm{G}, \mathcal{M}^{\prime \prime}\right) \quad \vdash_{\text {dread }}\left(\mathrm{G}, \mathcal{M}^{\prime \prime}\right)}{\mathcal{H}_{1},(\mathrm{G}, \mathcal{M}), \mathcal{H}_{2} \vdash_{\mathrm{b}}^{\mathcal{I}} \mathrm{G} \| \mathcal{M}^{\prime}} \mathcal{M}^{\prime} \equiv \mathcal{M} \cdot \mathcal{M}^{\prime \prime}
$$

where we have expanded the $\vdash_{\text {ok }}$ judgment. We say that a sequence $\mathcal{H}$ is coherent if $\mathcal{H}_{1} \vdash_{\mathrm{b}}^{\mathcal{I}} \mathrm{G} \| \mathcal{M}$ holds for any decomposition $\mathcal{H}=\mathcal{H}_{1},(\mathrm{G}, \mathcal{M}), \mathcal{H}_{2}$.

The proof is by coinduction on the definition of $\vdash_{\mathrm{b}} \mathrm{G} \| \mathcal{M}$ (see Figure 11). To this end, we define the set $\mathcal{A}$ as follows:

$$
\begin{aligned}
\widehat{\mathrm{G}} \| \widehat{\mathcal{M}} \in \mathcal{A} \text { if } \widehat{\mathcal{M}} \equiv_{\widehat{\mathrm{G}}} \mathcal{M}_{1} \cdot \mathcal{M}_{2} \text { and } \mathcal{H} \vdash_{\mathrm{b}}^{\mathcal{I}} \widehat{\mathrm{G}} \| \mathcal{M}_{1} \text { for some coherent } \mathcal{H} \\
\text { and } \vdash_{\text {agr }}\left(\widehat{\mathrm{G}}, \mathcal{M}_{2}\right) \text { and } \vdash_{\text {dread }}\left(\widehat{\mathrm{G}}, \mathcal{M}_{2}\right)
\end{aligned}
$$

From the hypothesis $\vdash_{\mathrm{b}}^{\mathcal{I}} \mathrm{G} \| \mathcal{M}$, we have immediately that $\mathrm{G} \| \mathcal{M} \in \mathcal{A}$, since $\mathcal{M} \equiv_{\mathrm{G}} \mathcal{M} \cdot \emptyset$, $\vdash_{\text {agr }}(\mathrm{G}, \emptyset)$ and $\vdash_{\text {dread }}(\mathrm{G}, \emptyset)$ always hold, and the empty sequence is coherent. Thus, to conclude the proof, we just have to show that $\mathcal{A}$ is consistent with respect to the rules in Figure 11. Then, we prove that for all coherent $\mathcal{H}, \widehat{\mathrm{G}}, \mathcal{M}_{1}$ and $\mathcal{M}_{2}$, if $\widehat{\mathcal{M}} \equiv_{\widehat{\mathrm{G}}} \mathcal{M}_{1} \cdot \mathcal{M}_{2}$ and $\mathcal{H} \vdash_{\mathrm{b}}^{\mathcal{I}} \widehat{\mathrm{G}} \| \mathcal{M}_{1}$ and $\vdash_{\text {agr }}\left(\widehat{\mathrm{G}}, \mathcal{M}_{2}\right)$ and $\vdash_{\text {dread }}\left(\widehat{\mathrm{G}}, \mathcal{M}_{2}\right)$, then $\vdash_{\mathrm{b}} \widehat{\mathrm{G}} \| \widehat{\mathcal{M}}$ is the conclusion of a rule in Figure 11 whose premises are in $\mathcal{A}$. The proof is by induction on the length of $\mathcal{H}$, splitting cases on the last rule used to derive $\mathcal{H} \vdash_{\mathrm{b}}^{\mathcal{I}} \widehat{\mathrm{G}} \| \mathcal{M}_{1}$.

[IB-END]: We have $\widehat{\mathrm{G}}=$ End and $\mathcal{M}_{1}=\emptyset$. By Lemma $6.3(4) \vdash_{\text {dread }}\left(\widehat{\mathrm{G}}, \mathcal{M}_{2}\right)$ implies $\vdash_{\text {read }}\left(\widehat{\mathrm{G}}, \mathcal{M}_{2}\right)$. From $\widehat{\mathrm{G}}=$ End we get $\mathcal{M}_{2}=\emptyset$, and so the statement is immediate by Rule [B-End].

[IB-CYCLE']: We have $\mathcal{H}=\mathcal{H}_{1},\left(\widehat{\mathrm{G}}, \mathcal{M}^{\prime}\right), \mathcal{H}_{2}$ and $\mathcal{M}_{1} \equiv \mathcal{M}^{\prime} \cdot \mathcal{M}^{\prime \prime}$ and $\vdash_{\text {agr }}\left(\widehat{\mathrm{G}}, \mathcal{M}^{\prime \prime}\right)$ and $\vdash_{\text {dread }}\left(\widehat{\mathrm{G}}, \mathcal{M}^{\prime \prime}\right)$, and, since $\mathcal{H}$ is coherent, we have $\mathcal{H}_{1} \vdash_{\mathrm{b}}^{\mathcal{I}} \widehat{\mathrm{G}} \| \mathcal{M}^{\prime}$ and $\mathcal{H}_{1}$ is coherent as well. Note that, since $\equiv_{\widehat{\mathrm{G}}}$ extends $\equiv$, we have $\widehat{\mathcal{M}} \equiv_{\widehat{\mathrm{G}}} \mathcal{M}_{1} \cdot \mathcal{M}_{2} \equiv_{\widehat{\mathrm{G}}} \mathcal{M}^{\prime} \cdot \mathcal{M}^{\prime \prime} \cdot \mathcal{M}_{2}$ and, by Lemma 6.6(1) and Lemma 6.6(2), we have $\vdash_{\text {agr }}\left(\widehat{\mathrm{G}}, \mathcal{M}^{\prime \prime} \cdot \mathcal{M}_{2}\right)$ and $\vdash_{\text {dread }}\left(\widehat{\mathrm{G}}, \mathcal{M}^{\prime \prime} \cdot \mathcal{M}_{2}\right)$. Then the statement follows by induction hypothesis, as $\mathcal{H}_{1}$ is strictly shorter than $\mathcal{H}$. [IB-Out]: We have $\widehat{\mathrm{G}}=\mathrm{pq} !\left\{\lambda_{i} ; \mathrm{G}_{i}\right\}_{i \in I}, \vdash_{\text {read }}\left(\widehat{\mathrm{G}}, \mathcal{M}_{1}\right)$ and $\mathcal{H},\left(\widehat{\mathrm{G}}, \mathcal{M}_{1}\right) \vdash_{\mathrm{b}}^{\mathcal{I}} \mathrm{G}_{i} \| \mathcal{M}_{1} \cdot\left\langle\mathrm{p}, \lambda_{i}, \mathrm{q}\right\rangle$ for all $i \in I$. We need to show that $\vdash_{\text {read }}(\widehat{\mathrm{G}}, \widehat{\mathcal{M}})$ and $\mathrm{G}_{i} \| \widehat{\mathcal{M}} \cdot\left\langle\mathrm{p}, \lambda_{i}, \mathrm{q}\right\rangle$ belongs to $\mathcal{A}$ for all $i \in I$, then the statement follows by Rule [B-OUT]. Note that $\vdash_{\text {read }}(\widehat{\mathrm{G}}, \widehat{\mathcal{M}})$ follows from $\widehat{\mathcal{M}} \equiv_{\widehat{\mathrm{G}}} \mathcal{M}_{1} \cdot \mathcal{M}_{2}$ and $\vdash_{\text {read }}\left(\widehat{\mathrm{G}}, \mathcal{M}_{1}\right)$ and $\vdash_{\text {dread }}\left(\widehat{\mathrm{G}}, \mathcal{M}_{2}\right)$ by Lemma $6.3(5)$ and Proposition 6.4(1). We now prove that $\mathrm{G}_{i} \| \widehat{\mathcal{M}} \cdot\left\langle\mathrm{p}, \lambda_{i}, \mathrm{q}\right\rangle$ belongs to $\mathcal{A}$ for all $i \in I$. The coherence of $\mathcal{H}$ implies the coherence of $\mathcal{H},\left(\widehat{\mathrm{G}}, \mathcal{M}_{1}\right)$. From $\vdash_{\text {agr }}\left(\widehat{\mathrm{G}}, \mathcal{M}_{2}\right)$ we get, for all $i \in I, \vdash_{\text {agr }}\left(\mathrm{G}_{i}, \mathcal{M}_{i}^{\prime}\right)$ for some $\mathcal{M}_{i}^{\prime}$ and $\mathcal{M}_{2} \cdot\left\langle\mathrm{p}, \lambda_{i}, \mathrm{q}\right\rangle \equiv_{\mathrm{G}_{i}}\left\langle\mathrm{p}, \lambda_{i}, \mathrm{q}\right\rangle \cdot \mathcal{M}_{i}^{\prime}$ by Lemma $6.5(1)$. From $\vdash_{\text {dread }}\left(\widehat{\mathrm{G}}, \mathcal{M}_{2}\right)$ we get $\vdash_{\text {dread }}\left(\mathrm{G}_{i}, \mathcal{M}_{2}\right)$ by Lemma $6.3(3)$ for all 


$$
\begin{aligned}
& \vdash_{\text {agr }}(\mathrm{G},\langle\mathrm{p}, p r, \mathrm{~s}\rangle) \quad \vdash_{\text {dread }}(\mathrm{G},\langle\mathrm{p}, p r, \mathrm{~s}\rangle) \quad \vdash_{\text {read }}(\mathrm{G}, \emptyset) \\
& \overline{\mathcal{H},(\mathrm{sp} ? k o ; \mathrm{ps} ! p r ; \mathrm{G},\langle\mathrm{s}, k o, \mathrm{p}\rangle),(\mathrm{ps} ! p r ; \mathrm{G}, \emptyset) \vdash_{\mathrm{b}}^{\mathcal{I}} \mathrm{G} \|\langle\mathrm{p}, p r, \mathrm{~s}\rangle} \\
& \frac{\mathcal{H},(\mathrm{sp} ? o k ; \mathrm{G},\langle\mathrm{s}, o k, \mathrm{p}\rangle) \vdash_{\mathrm{b}}^{\mathcal{I}} \mathrm{G} \| \emptyset}{\frac{\mathcal{H} \vdash_{\mathrm{b}}^{\mathcal{I}} \mathrm{sp} ? o k ; \mathrm{G} \|\langle\mathrm{s}, o k, \mathrm{p}\rangle}{\mathcal{H}}} \quad \frac{\mathcal{H},(\mathrm{sp} ? k o ; \mathrm{ps} ! p r ; \mathrm{G},\langle\mathrm{s}, k o, \mathrm{p}\rangle) \vdash_{\mathrm{b}}^{\mathcal{I}} \mathrm{ps} ! p r ; \mathrm{G} \| \emptyset}{\mathcal{H} \vdash_{\mathrm{b}}^{\mathcal{I}} \mathrm{sp} ? k o ; \mathrm{ps} ! p r ; \mathrm{G} \|\langle\mathrm{s}, k o, \mathrm{p}\rangle} \\
& \frac{(\mathrm{G}, \emptyset),\left(\mathrm{G}_{1},\langle\mathrm{p}, n d, \mathrm{~s}\rangle\right) \vdash_{\mathrm{b}}^{\mathcal{I}} \mathrm{G}_{2} \| \emptyset}{\frac{(\mathrm{G}, \emptyset) \vdash_{\mathrm{b}}^{\mathcal{I}} \mathrm{G}_{1} \|\langle\mathrm{p}, n d, \mathrm{~s}\rangle}{\vdash_{\mathrm{b}}^{\mathcal{I}} \mathrm{G} \| \emptyset}}
\end{aligned}
$$

where $\mathcal{H}=\left\{(\mathrm{G}, \emptyset),\left(\mathrm{G}_{1},\langle\mathrm{p}, n d, \mathrm{~s}\rangle\right),\left(\mathrm{G}_{2}, \emptyset\right)\right\}$

Figure 16: Inductive balancing of the hospital global type with the empty queue.

$$
\frac{\mathcal{H}^{\prime},(\mathrm{sp} ? o k ; \mathrm{G}, \mathcal{M}) \vdash_{\mathrm{agr}}(\mathrm{G}, \mathcal{M})}{\frac{\mathcal{H}^{\prime} \vdash_{\mathrm{agr}}(\mathrm{sp} ? o k ; \mathrm{G}, \mathcal{M})}{\frac{(\mathrm{G}, \mathcal{M}),\left(\mathrm{G}_{1}, \mathcal{M}\right) \vdash_{\mathrm{agr}}\left(\mathrm{G}_{2}, \mathcal{M}\right)}{\mathcal{H}^{\prime} \vdash_{\mathrm{agr}}(\mathrm{sp} ? k o ; \mathrm{ps} ! p r ; \mathrm{G}, \mathcal{M})}}}
$$

where $\mathcal{M}=\langle\mathrm{p}, \mathrm{pr}, \mathrm{s}\rangle$ and $\mathcal{H}^{\prime}=\left\{(\mathrm{G}, \mathcal{M}),\left(\mathrm{G}_{1}, \mathcal{M}\right),\left(\mathrm{G}_{2}, \mathcal{M}\right)\right\}$

Figure 17: Agreement of the hospital global type with the queue $\langle\mathrm{p}, p r, \mathrm{~s}\rangle$.

$i \in I$. Lemma 6.7 implies $\vdash_{\text {dread }}\left(G_{i}, \mathcal{M}_{i}^{\prime}\right)$ for all $i \in I$. From $\widehat{\mathcal{M}} \equiv_{\widehat{\mathrm{G}}} \mathcal{M}_{1} \cdot \mathcal{M}_{2}$ we have $\widehat{\mathcal{M}} \cdot\left\langle\mathrm{p}, \lambda_{i}, \mathrm{q}\right\rangle \equiv_{\mathrm{G}_{i}} \mathcal{M}_{1} \cdot\left\langle\mathrm{p}, \lambda_{i}, \mathrm{q}\right\rangle \cdot \mathcal{M}_{i}^{\prime}$ for all $i \in I$.

[IB-IN]: In this case we have $\widehat{\mathrm{G}}=\mathrm{pq} ?\left\{\lambda_{i} ; \mathrm{G}_{i}\right\}_{i \in I}, \vdash_{\text {read }}\left(\widehat{\mathrm{G}}, \mathcal{M}_{1}\right)$ and $\mathcal{M}_{1} \equiv\left\langle\mathrm{p}, \lambda_{h}, \mathrm{q}\right\rangle \cdot \mathcal{M}^{\prime}$ and $\mathcal{H},\left(\widehat{\mathrm{G}}, \mathcal{M}_{1}\right) \vdash \frac{\mathcal{I}}{\mathrm{b}} \mathrm{G}_{h} \| \mathcal{M}^{\prime}$ for some $h \in I$. We need to show that $\vdash_{\text {read }}(\widehat{\mathrm{G}}, \widehat{\mathcal{M}})$ and $\mathrm{G}_{h} \| \mathcal{M}^{\prime} \cdot \mathcal{M}_{2}$ belongs to $\mathcal{A}$, then the statement follows by Rule [B-IN]. Note that $\vdash_{\text {read }}(\widehat{\mathrm{G}}, \widehat{\mathcal{M}})$ follows from $\widehat{\mathcal{M}} \equiv \widehat{\mathrm{G}} \mathcal{M}_{1} \cdot \mathcal{M}_{2}$ and $\vdash_{\text {read }}\left(\widehat{\mathrm{G}}, \mathcal{M}_{1}\right)$ and $\vdash_{\text {dread }}\left(\widehat{\mathrm{G}}, \mathcal{M}_{2}\right)$ by Lemma 6.3(5) and Proposition 6.4(1). We now prove that $G_{h} \| \mathcal{M}^{\prime} \cdot \mathcal{M}_{2}$ belongs to $\mathcal{A}$. The coherence of $\mathcal{H}$ implies the coherence of $\mathcal{H},\left(\widehat{\mathrm{G}}, \mathcal{M}_{1}\right)$. From $\vdash_{\text {agr }}\left(\widehat{\mathrm{G}}, \mathcal{M}_{2}\right)$ we get $\vdash_{\text {agr }}\left(\mathrm{G}_{h}, \mathcal{M}_{2}\right)$ by Lemma $6.5(2)$. From $\vdash_{\text {dread }}\left(\widehat{\mathrm{G}}, \mathcal{M}_{2}\right)$ we get $\vdash_{\text {dread }}\left(\mathrm{G}_{h}, \mathcal{M}_{2}\right)$ by Lemma 6.3(3).

Finally, we observe that we can obtain a sound inductive version of weak balancing as well. We just have to remove premises involving readability and deep readability from the rules in Figure 13.

In Figures 16, 17, 18 and 19 we prove that the hospital global type with the empty queue is inductively balanced using the agreement, deep read and read judgments. Notice that in Figure 17 we need $\langle\mathrm{p}, p r, \mathrm{~s}\rangle \cdot\langle\mathrm{p}, n d, \mathrm{~s}\rangle \equiv_{\mathrm{G}_{1}}\langle\mathrm{p}, n d, \mathrm{~s}\rangle \cdot\langle\mathrm{p}, p r, \mathrm{~s}\rangle$. This holds since the messages $\langle\mathrm{p}, p r, \mathrm{~s}\rangle$ and $\langle\mathrm{p}, n d, \mathrm{~s}\rangle$ are $\mathrm{G}_{1}$-indistinguishable, being $\mathrm{G}_{1}=\mathrm{ps}$ ? $\left\{n d ; \mathrm{G}_{2}, p r ; \mathrm{G}_{2}\right\}$, where $\mathrm{G}_{2}$ does not contain different inputs from $\mathrm{p}$ to $\mathrm{s}$. 


$$
\begin{aligned}
& \frac{\vdash_{\text {read }}(\mathrm{G}, \mathcal{M})}{\mathrm{G}, \mathrm{G}_{1}, \mathrm{G}_{2}, \mathrm{sp} ? o k ; \mathrm{G} \vdash_{\text {dread }}(\mathrm{G}, \mathcal{M})} \\
& \mathrm{G}, \mathrm{G}_{1}, \mathrm{G}_{2} \vdash_{\text {dread }}(\mathrm{sp} \text { ? ok; G, } \mathcal{M}) \quad \mathrm{G}, \mathrm{G}_{1}, \mathrm{G}_{2} \vdash_{\text {dread }}(\mathrm{sp} \text { ? } k o ; \mathrm{ps} \text { ! pr; } \mathrm{G}, \mathcal{M}) \\
& \frac{\mathrm{G}, \mathrm{G}_{1} \vdash_{\text {dread }}\left(\mathrm{G}_{2}, \mathcal{M}\right)}{\frac{\mathrm{G}_{\text {dread }}\left(\mathrm{G}_{1}, \mathcal{M}\right)}{\vdash_{\text {dread }}(\mathrm{G}, \mathcal{M})}} \\
& \mathrm{G}, \mathrm{G}_{1}, \mathrm{G}_{2}, \mathrm{sp} \text { ?ko; ps!pr; G, ps!pr; } \vdash_{\text {dread }}(\mathrm{G}, \mathcal{M}) \\
& \mathrm{G}, \mathrm{G}_{1}, \mathrm{G}_{2}, \mathrm{sp} \text { ?ko; ps!pr; G } \vdash_{\text {dread }}(\mathrm{ps} ! p r ; \mathrm{G}, \mathcal{M})
\end{aligned}
$$

where $\mathcal{M}=\langle\mathrm{p}, p r, \mathrm{~s}\rangle$

Figure 18: Deep read of the hospital global type with the queue $\langle\mathrm{p}, p r, \mathrm{~s}\rangle$.

$$
\frac{\vdash_{\text {read }}\left(\mathrm{G}_{2}, \emptyset\right)}{\vdash_{\text {read }}\left(\mathrm{G}_{1},\langle\mathrm{p}, p r, \mathrm{~s}\rangle\right)}
$$

Figure 19: Read of the hospital global type with the queue $\langle\mathrm{p}, p r, \mathrm{~s}\rangle$.

\section{RELATED AND FUTURE WORKS}

Since their introduction in [HYC08] multiparty sessions have been equipped with global types. The literature on multiparty sessions is very large, see $\left[\mathrm{HLV}^{+} 16\right]$ for a survey. In almost all papers the building blocks of global types are synchronous communications between senders and receivers. This does not fit well with asynchronous communications. To fill this gap a suitable subtyping is proposed in [MYH09]. This subtyping enjoys completeness $\left[\mathrm{GPP}^{+} 21\right]$, i.e. any extension of it would be unsound. Unfortunately the obtained type system is not effective, since this subtyping is undecidable [BCZ17, LY17]. Decidable restrictions of this subtyping relation have been proposed [BCZ17, LY17, BCZ18]. In particular, subtyping is decidable when both internal and external choices are forbidden in one of the two compared processes [BCZ17]. This result is improved in [BCZ18], where both the subtype and the supertype can contain either internal or external choices. More interestingly, the work $\left[\mathrm{BCL}^{+} 21\right]$ presents a sound (though not complete) algorithm for checking asynchronous subtyping for binary sessions. It is worthwhile to notice that Example 3.21 of $\left[\mathrm{BCL}^{+} 21\right]$ is typable in our system with a provably balanced global type. Instead, in standard type systems it requires a subtyping which is not derivable by the algorithm in $\left[\mathrm{BCL}^{+} 21\right]$. In our notation this example is $\mathrm{p} \llbracket P \rrbracket \| \mathrm{q} \llbracket Q \rrbracket$ where $P=\mathrm{q} ?\left\{\lambda_{1} ; P_{1}, \lambda_{2} ; P_{2}\right\}$, $P_{1}=\mathrm{q} ! \lambda ; \mathrm{q} ! \lambda ; \mathrm{q} ! \lambda ; P, P_{2}=\mathrm{q} ! \lambda ; P_{2}$ and $Q=\mathrm{p} !\left\{\lambda_{1} ; Q_{1}, \lambda_{2} ; Q_{2}\right\}, Q_{1}=\mathrm{p} ? \lambda ; Q, Q_{2}=\mathrm{p} ? \lambda ; Q_{2}$. The reduction of this network has a loop in which for each cycle the number of messages $\langle p, \lambda, q\rangle$ in the queue increases by two. Our type inference algorithm gives between others the global type $\mathrm{G}=\mathrm{q} p !\left\{\lambda_{1} ; \mathrm{q} p\right.$ ? $\lambda_{1} ; \mathrm{G}_{1}, \lambda_{2} ; \mathrm{q} p$ ? $\left.\lambda_{2} ; \mathrm{G}_{2}\right\}$, where $\mathrm{G}_{1}=\mathrm{pq} \mathrm{q} \lambda ; \mathrm{pq} ! \lambda ; \mathrm{pq}$ ! $\lambda ; \mathrm{pq} ? \lambda ; \mathrm{G}$ and $\mathrm{G}_{2}=\mathrm{pq}$ ! $\lambda ; \mathrm{pq} ? \lambda ; \mathrm{G}_{2}$.

To the best of our knowledge global types with split outputs and inputs are considered only in [CDG21, DGD21, CDG22]. The above network can be typed in [DGD21] and in [CDG22], but not in [CDG21]. In [CDG21, DGD21] there is no choice of inputs and the running example of the current paper cannot be typed. Instead [CDG22] further extends the 
syntax of global types by allowing multiple senders in internal choices and multiple receivers in external choices. The obtained typing is undecidable and it does not ensure the absence of orphan messages. The present work springs from [DGD21], but with many improvements:

- there are choices of inputs in global types;

- networks are typed by global types, while in all other papers, but [CDG22], either global types are projected onto local types and local types are assigned to processes or global types are projected onto processes;

- the proof that the type system allows us to type an arbitrary network is new;

- the type inference algorithm is new and very simple compared for example to the algorithm in [GHH21], essentially thanks to the flexible syntax of global types;

- the conditions for taming types are more permissive: this also is due to the new syntax of global types;

- the proof of undecidability for the (weak) balancing predicate is original also if inspired by the proof in [BCZ17];

- the decision algorithm for balancing is empowered by the use of the equivalence on queues parametrised on global types.

The type system of [DGD21] is implemented in co-logic programming, see [BD21a]. The tool is available at [BD21b]. We plan to extend this implementation to the present type system.

As future work we want to compare typability in our type system with typability in the standard type system enriched by asynchronous subtyping.

Acknowledgment. We are grateful to Ilaria Castellani and Elena Zucca for enlightening discussions on the subject of this paper. We are strongly indebted to the anonymous referees since thanks to their constructive remarks the current version of the paper greatly improves the submitted one.

\section{REFERENCES}

[AD15] Davide Ancona and Agostino Dovier. A theoretical perspective of coinductive logic programming. Fundamenta Informaticae, 140(3-4):221-246, 2015. doi:10.3233/FI-2015-1252.

[AMV06] Jirí Adámek, Stefan Milius, and Jiri Velebil. Iterative algebras at work. Mathematical Structures in Computer Scienc, 16(6):1085-1131, 2006. doi:10.1017/S0960129506005706.

$\left[\mathrm{BCD}^{+} 08\right]$ Lorenzo Bettini, Mario Coppo, Loris D'Antoni, Marco De Luca, Mariangiola Dezani-Ciancaglini, and Nobuko Yoshida. Global progress in dynamically interleaved multiparty sessions. In Franck van Breugel and Marsha Chechik, editors, CONCUR, volume 5201 of LNCS, pages 418-433. Springer, 2008. doi:10.1007/978-3-540-85361-9\_33.

$\left[\mathrm{BCL}^{+} 21\right]$ Mario Bravetti, Marco Carbone, Julien Lange, Nobuko Yoshida, and Gianluigi Zavattaro. A sound algorithm for asynchronous session subtyping and its implementation. Logical Methods in Computer Science, 17(1):20:1-20:35, 2021.

[BCZ17] Mario Bravetti, Marco Carbone, and Gianluigi Zavattaro. Undecidability of asynchronous session subtyping. Information and Computation, 256:300-320, 2017. doi:10.1016/j.ic.2017.07.010.

[BCZ18] Mario Bravetti, Marco Carbone, and Gianluigi Zavattaro. On the boundary between decidability and undecidability of asynchronous session subtyping. Theoretical Computer Science, 722:19-51, 2018. doi:10.1016/j.tcs.2018.02.010.

[BD21a] Riccardo Bianchini and Francesco Dagnino. Asynchronous global types in co-logic programming. In Ferruccio Damiani and Ornela Dardha, editors, Coordination, volume 12717 of LNCS, pages 134-146. Springer, 2021.

[BD21b] Riccardo Bianchini and Francesco Dagnino. Queryagt. https://github.com/RiccardoBianc/ QueryAGT, 2021. 
[CDCYP16] Mario Coppo, Mariangiola Dezani-Ciancaglini, Nobuko Yoshida, and Luca Padovani. Global progress for dynamically interleaved multiparty sessions. Mathematical Structures in Computer Science, 26(2):238-302, 2016. doi:10.1017/S0960129514000188.

[CDG21] Ilaria Castellani, Mariangiola Dezani-Ciancaglini, and Paola Giannini. Global types and event structure semantics for asynchronous multiparty sessions. CoRR, abs/2102.00865, 2021. URL: https://arxiv.org/abs/2102.00865.

[CDG22] Ilaria Castellani, Mariangiola Dezani-Ciancaglini, and Paola Giannini. Asynchronous sessions with input races. In Marco Carbone and Rumyana Neykova, editors, PLACES, volume 356 of EPTCS, pages 12-23. Open Publishing Association, 2022.

[Cou83] Bruno Courcelle. Fundamental properties of infinite trees. Theoretical Computer Science, 25:95169, 1983. doi:10.1016/0304-3975(83)90059-2.

[Dag21] Francesco Dagnino. Foundations of regular coinduction. Logical Methods in Computer Science, 17:2:1-2:29, 2021. doi :10.46298/lmcs-17 (4:2) 2021.

$\left[\right.$ DCGJ $^{+}$16] Mariangiola Dezani-Ciancaglini, Silvia Ghilezan, Svetlana Jaksic, Jovanka Pantovic, and Nobuko Yoshida. Precise subtyping for synchronous multiparty sessions. In Simon Gay and Jade Alglave, editors, PLACES, volume 203 of EPTCS, pages 29 - 44. Open Publishing Association, 2016. doi:10.4204/EPTCS.203.3.

[DGD21] Francesco Dagnino, Paola Giannini, and Mariangiola Dezani-Ciancaglini. Deconfined global types for asynchronous sessions. In Ferruccio Damiani and Ornela Dardha, editors, COORDINATION, volume 12717 of $L N C S$, pages 41-60. Springer, 2021.

[DH12] Romain Demangeon and Kohei Honda. Nested protocols in session types. In Maciej Koutny and Irek Ulidowski, editors, CONCUR, volume 7454 of LNCS, pages 272-286. Springer, 2012. doi:10.1007/978-3-642-32940-1\_20.

[DY11] Pierre-Malo Deniélou and Nobuko Yoshida. Dynamic multirole session types. In Mooly Sagiv Thomas Ball, editor, POPL, pages 435-446. ACM Press, 2011. doi:10.1145/1926385.1926435.

[GH05] Simon Gay and Malcolm Hole. Subtyping for session types in the pi calculus. Acta Informatica, 42(2/3):191-225, 2005. doi:10.1007/s00236-005-0177-z.

[GHH21] Rob van Glabbeek, Peter Höfner, and Ross Horne. Assuming just enough fairness to make session types complete for lock-freedom. In Leonid Libkin, editor, LICS, pages 1-13. IEEE, 2021.

[GJP $\left.{ }^{+} 19\right] \quad$ Silvia Ghilezan, Svetlana Jaksic, Jovanka Pantovic, Alceste Scalas, and Nobuko Yoshida. Precise subtyping for synchronous multiparty sessions. Journal of Logic and Algebraic Methods in Programming, 104:127-173, 2019. doi:10.1016/j.jlamp.2018.12.002.

$\left[\mathrm{GPP}^{+} 21\right]$ Silvia Ghilezan, Jovanka Pantović, Ivan Prokić, Alceste Scalas, and Nobuko Yoshida. Precise subtyping for asynchronous multiparty sessions. Proceedings of the ACM on Programming Languages, 5(POPL):1-28, 2021. doi:10.1145/3434297.

$\left[\mathrm{HLV}^{+} 16\right]$ Hans Hüttel, Ivan Lanese, Vasco T. Vasconcelos, Luís Caires, Marco Carbone, Pierre-Malo Deniélou, Dimitris Mostrous, Luca Padovani, António Ravara, Emilio Tuosto, Hugo Torres Vieira, and Gianluigi Zavattaro. Foundations of session types and behavioural contracts. ACM Computing Surveys, 49(1):3:1-3:36, 2016.

[HYC08] Kohei Honda, Nobuko Yoshida, and Marco Carbone. Multiparty asynchronous session types. In George C. Necula and Philip Wadler, editors, POPL, pages 273-284. ACM Press, 2008. doi:10.1145/1328897.1328472.

[HYC16] Kohei Honda, Nobuko Yoshida, and Marco Carbone. Multiparty asynchronous session types. Journal of ACM, 63(1):9:1-9:67, 2016. doi:10.1145/2827695.

[Koz97] Dexter Kozen. Automata and computability. Undergraduate texts in computer science. Springer, 1997.

[LW94] Barbara Liskov and Jeannette M. Wing. A behavioral notion of subtyping. ACM Transactions on Programming Languages and Systems, 16(6):1811-1841, 1994. doi:10.1145/197320.197383.

[LY17] Julien Lange and Nobuko Yoshida. On the undecidability of asynchronous session subtyping. In Javier Esparza and Andrzej S. Murawski, editors, FOSSACS, volume 10203 of LNCS, pages 441-457, 2017. doi:10.1007/978-3-662-54458-7\_26.

[MYH09] Dimitris Mostrous, Nobuko Yoshida, and Kohei Honda. Global principal typing in partially commutative asynchronous sessions. In Giuseppe Castagna, editor, ESOP, volume 5502 of LNCS, pages 316-332. Springer, 2009. doi:10.1007/978-3-642-00590-9\_23. 
[SBMG07] Luke Simon, Ajay Bansal, Ajay Mallya, and Gopal Gupta. Co-logic programming: Extending logic programming with coinduction. In Lars Arge, Christian Cachin, Tomasz Jurdzinski, and Andrzej Tarlecki, editors, ICALP, volume 4596 of $L N C S$, pages 472-483. Springer, 2007.

[SDC19] Paula Severi and Mariangiola Dezani-Ciancaglini. Observational equivalence for multiparty sessions. Fundamenta Informaticae, 167:267-305, 2019. doi:10.1007/s00236-019-00332-y.

[Sim06] Luke Simon. Extending logic programming with coinduction. PhD thesis, University of Texas at Dallas, 2006.

[SMBG06] Luke Simon, Ajay Mallya, Ajay Bansal, and Gopal Gupta. Coinductive logic programming. In Sandro Etalle and Miroslaw Truszczynski, editors, ICLP, volume 4079 of LNCS, pages 330-345. Springer, 2006. 